\title{
The Behaviour of Solutions with Singularities on a Characteristic Surface to Linear Partial Differential Equations in the Complex Domains
}

\author{
By \\ Sunao ŌUCHF*
}

\section{§ 0. Introduction}

Let $L\left(z, \partial_{z}\right)$ be a linear partial differential operator defined in a neighbourhood $\Omega$ of $z=0$ in $C^{n+1}$. Its coefficients are holomorphic in $\Omega$. Let $K$ be a connected nonsingular complex hypersurface through $z=0$ and characteristic for $L\left(z, \partial_{z}\right)$. We choose the coordinate so that $K=\left\{z \in \Omega ; z_{0}=0\right\}$. In the present paper we study the equation

$$
L\left(z, \partial_{z}\right) u(z)=f(z),
$$

where $u(z)$ and $f(z)$ are holomorphic in a sector $\Omega\left(\theta_{0}\right)$ whose edge is $K, \Omega\left(\theta_{0}\right)$ $=\left\{z \in \Omega-\left\{z_{0}=0\right\} ;\left|\arg z_{0}\right|<\theta_{0}\right\}$. It is the main purpose of this paper to show under some conditions on $L\left(z, \partial_{z}\right)$ that if $u(z)$ has at most some exponential growth on $\Omega\left(\theta_{0}\right)$ and $f(z)$ has an asymptotic expansion with bounds on $\Omega\left(\theta_{0}\right)$ as $z_{0} \rightarrow 0$, then $u(z)$ has also an asymptotic expansion of the same type as $f(z)$ (Theorems 1.5 and 1.7). The conditions on $L\left(z, \partial_{z}\right)$ are given by means of characteristic indices $\left\{\sigma_{i}: 0 \leqq i \leqq p\right\}$ of $K$ and the localizations on $K$ defined in [9]. The growth order of $u(z)$ and the asymptotic expansion of $f(z)$ are characterized by $\sigma_{p-1}$. When $f(z)$ has no singularities on $K$, that is, it is holomorphic in $\Omega$, the equation (0.1) was investigated in [12] and it was shown that $u(z)$ is also holomorphic in $\Omega$ under some conditions, which is contained in the results in this paper.

As for existence of solutions of (0.1) which are singular on $K$ were investigated in many papers, for example, [1], [2], [3], [5], [10], [13], [15] and papers cited there.

In $\S 1$ we give notations and definitions of characteristic indices and function spaces, and state the results. Theorems 1.11 and 1.13 are applied to the

Communicated by M. Kashiwara, December 4, 1991.

1991 Mathematics Subject Classification: 35A20, 35C15, 35C20

* Department of Mathematics, Sophia University, Tokyo 102, Japan 
proofs of Theorems 1.5 and 1.7. In $\S 2$ we study the function spaces introduced in $\S 1$. In $\S 3$ we introduce an integro-differential operator $\mathcal{L}_{\alpha}\left(z, \lambda, \zeta, \partial_{z}\right.$, $\left.\partial_{\zeta}, \lambda t_{0}-\lambda \partial_{\lambda}\right), 0<\alpha<1$, which is derived from $L\left(z, \partial_{z}\right)$, and construct a formal solution $V(z, t, \lambda)$ of an equation

$$
\mathcal{L}_{\alpha}\left(z, \lambda, \zeta, \partial_{z}, \partial_{\zeta}, \lambda t_{0}-\lambda \partial_{\lambda}\right) V(z, t, \lambda, \zeta)=F(z, t, \lambda) f_{n_{0}}\left(\zeta+\tau z_{1}\right) .
$$

In $\S 4$ we construct the kernel functions $G(\psi ; w, z, t)$ and $G_{R}(\psi ; w, z, t)$ in Theorem 1.11, where we use $\mathcal{L}_{\alpha_{p-1}}\left(z, \lambda, \zeta, \partial_{z}, \partial_{\zeta}, \lambda t_{0}-\lambda \partial_{\lambda}\right), \alpha_{p-1}=\left(\sigma_{p-1}-1\right) / \sigma_{p-1}$. In $\S 5$ we investigate integral operators acting on holomorphic functions on a sector and give the proof of Theorem 1.11. It is the main purpose in $\S 6$ to give Theorem 6.28 which is used to show Theorem 1.5 in $\S 7$. We give an integral representation of $u(z)$ in $(0.1)$ in order to prove Theorem 6.28 , where $\mathcal{L}_{a_{1}}\left(z, \lambda, \zeta, \partial_{z}, \partial_{\zeta}, \lambda t_{0}-\lambda \partial_{\lambda}\right), \alpha_{1}=\left(\sigma_{1}-1\right) / \sigma_{1}$, is used. The representation in this paper is somewhat different from that in [6] and [7], and sufficient for our purpose. The arguments in $\S 6$ are similar to those in [12]. But we investigate the equations under the weaker conditions than in [12]. We used the operator $\mathcal{L}_{\alpha}\left(z, \lambda, \zeta, \partial_{z}, \partial_{\zeta}\right)$ in $[12]$, which does not contain $\lambda t_{0}-\lambda \partial_{\lambda}$. Since we treat $(0.2)$ in this paper, the arguments become somewhat complicated. In $\S 7$ firstly we summerize about majorant functions and show Theorem 1.13. Next we give the proofs of Theorems 1.5 and 1.7. We make use of Theorems 1.11 and 6.28 in the proof of Theorem 1.5, and Theorem 1.7 follows from Theorems 1.5 and 1.13. Finally we give the proofs of estimates, that is, the proofs of Propositions 4.1 and 6.8, which are assumed in the preceding arguments. Proposition 4.1 (Proposition 6.8) gives estimates of functions appearing in construction of $G(w, z, t)$ (resp. in the representation of $u(z)$ ). Many constants will appear in this paper. So for simplicity we denote various constants by the same notations $A, B, C$, etc..

\section{$\S 1$. Notations and Definitions}

The following usual notations are used: $z=\left(z_{0}, z_{1}, \cdots, z_{n}\right)=\left(z_{0}, z_{1}, z^{\prime \prime}\right)=$ $\left(z_{0}, z^{\prime}\right)$ is an element of $C^{n+1}$, while $\xi=\left(\xi_{0}, \xi_{1}, \xi^{\prime \prime}\right)=\left(\xi_{0}, \xi^{\prime}\right)$ is the variable dual to $z, \partial_{i}=\partial / \partial_{z_{\imath}}$ and $\partial_{z}=\left(\partial_{0}, \partial_{1}, \cdots, \partial_{n}\right)=\left(\partial_{0}, \partial_{1}, \partial^{\prime \prime}\right)=\left(\partial_{0}, \partial^{\prime}\right)$. $|z|=\max \left\{\left|z_{i}\right| ; 0 \leqq i \leqq n\right\}$. $N=\{1,2,3, \cdots\}$ and $\mathbb{Z}^{+}=\{0,1,2, \cdots\}$. For a real number $a$, [a] means the integral part of $a$. Let $K$ be a nonsingular complex hypersurface through $z=0$. We choose the coordinate so that $K=\left\{z_{0}=0\right\}$ to simplify the statements. In order to give the results we define firstly the characteristic indices $\left\{\sigma_{\imath}\right\}(0 \leqq i \leqq p)$ for a linear partial differential operator $L\left(z, \partial_{z}\right)$ of order $m \geqq 1$ and with the holomorphic coefficients in a neighbourhood of $z=0$, and secondly function spaces. The characteristic indices were introduced in [9] and [10]. We write $L\left(z, \partial_{z}\right)$ in the following: 


$$
\left\{\begin{array}{l}
L\left(z, \partial_{z}\right)=\sum_{k=0}^{m} L_{k}\left(z, \partial_{z}\right), \\
L_{k}\left(z, \partial_{z}\right)=\sum_{l=s_{k}}^{k} A_{k, l}\left(z, \partial^{\prime}\right)\left(\partial_{0}\right)^{k-l},
\end{array}\right.
$$

where $L_{k}\left(z, \partial_{z}\right)$ is the homogeneous part of the order $k, A_{k, s_{k}}\left(z, \partial^{\prime}\right) \not \equiv 0$ if $L_{k}\left(z, \partial_{z}\right) \not \equiv 0$, and otherwise we put $s_{k}=+\infty$. By expanding $A_{k, l}\left(z, \partial^{\prime}\right)(\not \equiv 0)$ with respect to $z_{0}, A_{k, l}\left(z, \partial^{\prime}\right)=\sum_{j=j(k, l)}^{-\infty} z_{0}^{j} a_{k, l}^{j}\left(z^{\prime}, \partial^{\prime}\right)=z_{0}^{j(k, l)} a_{k, l}\left(z, \partial^{\prime}\right)$, where $a_{k, l}^{j(k, l)}\left(z^{\prime}, \partial^{\prime}\right) \equiv \equiv$. We put conventionally $j(k, l)=+\infty$ if $A_{k, l}\left(z, \partial^{\prime}\right) \equiv 0$. We have

$$
\begin{aligned}
L_{k}\left(z, \partial_{z}\right) & =\sum_{l=s_{k}}^{k} z_{0}^{j(k, l)} a_{k, l}\left(z, \partial^{\prime}\right)\left(\partial_{0}\right)^{k-l} \\
& =\sum_{d \geq d_{k}}\left(\sum_{l+j(k, l)=d} z_{0}^{j(k, l)} a_{k, l}\left(z, \partial^{\prime}\right)\left(\partial_{0}\right)^{k-l}\right),
\end{aligned}
$$

where $d_{k}=\min _{l}\left\{d_{k, l}=l+j(k, l)\right\}$, and $d_{k}=+\infty$ if $L_{k}\left(z, \partial^{\prime}\right) \equiv 0$. If $d_{k}<+\infty$, put

$$
\left\{\begin{array}{l}
l_{k}=\max \left\{l ; l+j(k, l)=d_{k}, \quad a_{k, l}\left(z, \partial^{\prime}\right) \not \equiv 0\right\}, \\
j_{k}=d_{k}-l_{k} .
\end{array}\right.
$$

Obviously $l_{k} \geqq s_{k}$. When $l+j(k, l)>d_{k}$, we do not have to expand $A_{k, l}(z, \partial)$ with respect to $z_{0}$ up to $j(k, l)$. Put $j^{\prime}(k, l)=d_{k}+1-l$. Then we have $A_{k, l}(z, \partial)$ $=z_{0}^{j^{\prime}(k, l)} a_{k, l}^{\prime}\left(z, \partial^{\prime}\right)\left(\partial_{0}\right)^{k-l}$ and use this expression in (1.2). So, when $l+j(k, l)>d_{k}$, we put $j(k, l)=j^{\prime}(k, l)$ and $a_{k, l}\left(z, \partial^{\prime}\right)=a_{k, l}^{\prime}\left(z, \partial^{\prime}\right)$. Let us define the characteristic indices $\left\{\sigma_{i} ; 0 \leqq i \leqq p\right\}$ : Consider the set $A=\left\{\left(k, d_{k}\right) \in \boldsymbol{R}^{2} ; 0 \leqq k \leqq m, d_{k} \neq+\infty\right\}$ and its convex hull $\hat{A}$. Let $\Sigma$ be the lower convex part of the boundary of $\hat{A}$. In general $\Sigma$ consists of segments $\Sigma(\imath)\left(1 \leqq i \leqq p^{\prime}\right)$ and let $\Delta$ be the set of vertices of $\Sigma, \Delta=\left\{\left(k_{i}, d_{k_{i}}\right) \in \boldsymbol{R}^{2} ; i=0,1, \cdots, p^{\prime}\right\}, m=k_{0}>k_{1}>\cdots>k_{p^{\prime}} \geqq 0$ (see Fig. 1.1). We define as in [9] and [10],

$$
\left.\sigma_{i}=\max \left\{1, d_{k_{i-1}}-d_{k_{i}}\right) /\left(k_{i-1}-k_{i}\right)\right\} .
$$

Then there is a $p \in N, p \leqq p^{\prime}+1$, such that $\sigma_{1}>\sigma_{2}>\cdots>\sigma_{p-1}>1=\sigma_{p}$, and we put $\sigma_{0}=+\infty$. If $\Sigma$ consists of one point $\left(m, d_{m}\right)$, put $\sigma_{1}=1$.

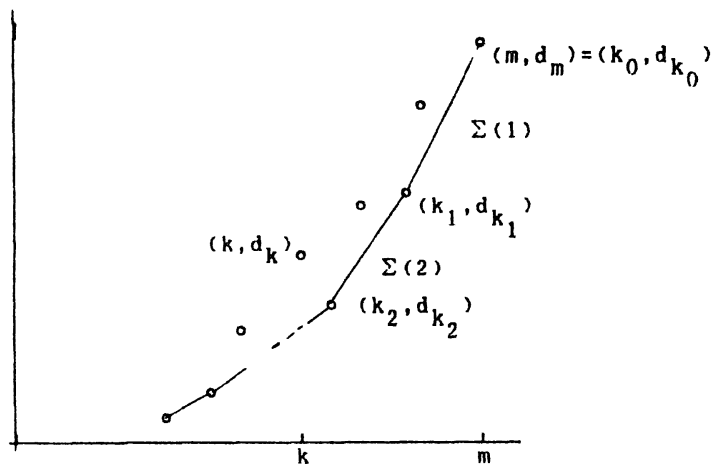

Fig. 1.1

We put $\Sigma(0)=\left\{\left(m, d_{m, l}\right) \in \boldsymbol{R}^{2} ; d_{m_{\mathrm{o}} l} \neq+\infty\right\}$. We note that 


$$
\prod_{i=0}^{p-1} a_{k_{i}, l k_{i}}\left(0, z^{\prime}, \xi^{\prime}\right) \neq 0 \text {. }
$$

Definition 1.1. We call $\left\{\sigma_{i} ; 0 \leqq i \leqq p\right\}$ the characteristic indices of the sur face $K$ for $L\left(z, \partial_{z}\right)$.

Let us define function spaces. For a set $A \subset C^{N}, \tilde{A}$ is the universal covering space of $A . \quad \mathcal{O}(A)(\mathcal{O}(\tilde{A}))$ is the set of all holomorphic functions on $A$ (resp. $\tilde{A})$. $O(\tilde{A})$ contains multi-valued holomorphic functions on $A$. Let $\Omega=\Omega_{0} \times \Omega^{\prime}$ be an open polydisk in $C^{n+1}$, where $\Omega_{0}=\left\{z_{0} \in C^{1} ;\left|z_{0}\right|<r_{0}\right\}$ and $\Omega^{\prime} \subset C^{n}$. Put $\Omega_{0}(a, b)=\left\{z \in \widetilde{\Omega_{0}-\{0\}} ; a<\arg z_{0}<b\right\}$ and $\Omega((a, b), D)=\Omega_{0}(a, b) \times D$ for $D \Subset \Omega^{\prime}$, where $D \Subset \Omega^{\prime}$ means that $\bar{D}$ is compact and $\bar{D} \subset \Omega^{\prime}$. We simply denote $\Omega_{0}(a, b)$ $\times \Omega^{\prime}$ by $\Omega(a, b)$ and $\Omega(-a, a), a>0$, by $\Omega(a)$. $\mathcal{O}(\Omega(a, b))$ contains multi-valued functions on $\Omega-K$, if $b-a>2 \pi$. We have $\mathcal{O}(\widetilde{\Omega-K})=\mathcal{O}(\Omega(-\infty,+\infty))$. We remark that the notations in this paper are different from those in [11], for example, $\mathcal{O}(\widetilde{\Omega-K})(\mathcal{O}(\Omega(a)))$ was denoted by $\widetilde{\mathcal{O}}(\Omega-K)$ (resp. $\left.\widetilde{\mathcal{O}}\left(\Omega_{a}\right)\right)$ in $[11]$. In the following the center of $\Omega$ is the origin.

Definition 1.2. For $\kappa, h>0, \mathcal{O}_{\{\kappa\}}, h(\Omega(a, b))$ is the set of all $f(z) \in \mathcal{O}(\Omega(a, b))$ such that for any $a^{\prime}, b^{\prime}\left(a<a^{\prime}<b^{\prime}<b\right)$ and any $D \Subset \Omega^{\prime}$

$$
|f(z)| \leqq A \exp \left(h\left|z_{0}\right|^{-k}\right) \quad \text { in } \Omega\left(\left(a^{\prime}, b^{\prime}\right), D\right)
$$

for a constant $A=4\left(a^{\prime}, b^{\prime}, D\right)$. We put $\mathcal{O}_{(\kappa)}(\Omega(a, b))=\bigcap_{h>0} \mathcal{O}_{\{\kappa\}, h}(\Omega(a, b))$.

$f(z) \in \mathcal{O}_{(x)}(\Omega(a, b))$ if and only if for any $a^{\prime}, b^{\prime}\left(a<a^{\prime}<b^{\prime}<b\right)$, any $\varepsilon>0$ and any $D \Subset \Omega^{\prime}$

$$
|f(z)| \leqq A_{\varepsilon, a^{\prime}, b^{\prime}, D} \exp \left(\varepsilon\left|z_{0}\right|^{-\kappa}\right) \quad \text { in } \Omega\left(\left(a^{\prime}, b^{\prime}\right), D\right) .
$$

Function spaces introduced in the following are characterized by the behaviour of functions near $K=\left\{z_{0}=0\right\}$.

Definition 1.3. $\operatorname{Asy}_{\{k\}}(\Omega(a, b)), 0<\kappa \leqq+\infty$, is the class of all $f(z) \in \mathcal{O}(\Omega(a, b))$ having the asymptotic expansion of the following form as $z_{0} \rightarrow 0$ : for any $N \geqq 1$

$$
\left|f(z)-\sum_{k=0}^{N-1} a_{k}\left(z^{\prime}\right) z_{0}^{k}\right| \leqq A B^{N} \Gamma(N / \kappa+1)\left|z_{0}\right|^{N}
$$

in any $\Omega\left(\left(a^{\prime}, b^{\prime}\right), D\right)\left(a<a^{\prime}<b^{\prime}<b, D \Subset \Omega^{\prime}\right)$, where $a_{k}\left(z^{\prime}\right) \in \mathcal{O}\left(\Omega^{\prime}\right), A$ and $B$ are constants depending on $\Omega\left(\left(a^{\prime}, b^{\prime}\right), D\right) . \quad f(z) \in A s y_{(k)\}}(\Omega(a, b))$ is said to have the $\kappa$-asymptotic expansion in $\Omega(a, b)$.

We note that $f(z) \in A s y_{1+\infty)}(\Omega(a, b))$ means $f(z) \in \mathcal{O}(\Omega)$.

Definition 1.4. (1) $\tilde{\mathbb{M}}-a s y_{\{\kappa\}}(\Omega(a, b)), 0<\kappa \leqq+\infty$, is the class of all $f(z) \in$ $\mathcal{O}(\Omega(a, b))$ having the following asymptotic expansion in the form with polar and logarithmic terms as $z_{0} \rightarrow 0$ : for any $N \geqq 1$ 


$$
\begin{aligned}
& \left|f(z)-\sum_{k=0}^{N-1} a_{k}\left(z^{\prime}\right) z_{0}^{k} \log z_{0}-\sum_{k=-H}^{N-1} b_{k}\left(z^{\prime}\right) z_{0}^{k}\right| \\
& \quad \leqq A B^{N} \Gamma(N / \kappa+1)\left|z_{0}\right|^{N}\left|\log z_{0}\right|, \quad \text { and } \\
& \left|f(z)-\sum_{k=0}^{N} a_{k}\left(z^{\prime}\right) z_{0}^{k} \log z_{0}-\sum_{k=-H}^{N-1} b_{k}\left(z^{\prime}\right) z_{0}^{k}\right| \\
& \quad \leqq A B^{N} \Gamma(N / \kappa+1)\left|z_{0}\right|^{N}
\end{aligned}
$$

hold in any $\Omega\left(\left(a^{\prime}, b^{\prime}\right), D\right)\left(a<a^{\prime}<b^{\prime}<b, D \in \Omega^{\prime}\right)$, where $H \in Z_{+}$and $a_{k}\left(z^{\prime}\right), b_{k}\left(z^{\prime}\right)$ $\in \mathcal{O}\left(\Omega^{\prime}\right)$. $\quad A$ and $B$ are constants depending on $a^{\prime}, b^{\prime}$ and $D$.

(2) $\tilde{\mathscr{N}}(\Omega-K)$ is the set of all $f(z) \in \mathcal{O}(\widetilde{\Omega-K})$ having at most polar or logarithmic singularities on $K$, that is, $f(z)=a(z) \log z_{0}+b(z) / z_{0}^{H}, a(z), \quad b(z) \in$ $\mathcal{O}(\Omega), H \in \boldsymbol{Z}^{+}$.

We note that if $f(z) \in \widetilde{C}-a s y_{(\kappa)}(\Omega(a, b))$ and $b-a \leqq \pi / \kappa$, then there are $a(z)$, $b(z) \in A s y_{(\kappa)}(\Omega(a, b))$ such that $f(z)=a(z) \log z_{0}+b(z) / z_{0}^{H}$ (see Proposition 2.1). We have $\tilde{\mathscr{M}}$-as $y_{1+\infty)}(\Omega(a, b))=\widetilde{\mathscr{M}}(\Omega-K)$.

Now let us state the main results.

Theorem 1.5. Suppose that $L\left(z, \partial_{2}\right)$ satisfies the conditions
(a) $\sigma_{1}>1$,
(b) $d_{k_{p-1}}=0$,
(c) $d_{k_{1}}=s_{k_{i}}$ for $0 \leqq i \leqq p-2$.

Let $\theta_{0}$ be an arbitrary positive constant and $u(z) \in \mathcal{O}\left(\Omega\left(\theta_{0}\right)\right)$ be a solution of

$$
L\left(z, \partial_{z}\right) u(z)=f(z) \in A s y_{(\kappa)}\left(\Omega\left(\theta_{0}\right)\right),
$$

where $0<\kappa \leqq \gamma=\sigma_{p-1}-1$. If $u(z) \in \mathcal{O}_{(\gamma)}\left(\Omega\left(\theta_{0}\right)\right)$, then $u(z) \in A s y_{(\kappa)}\left(\Omega\left(\theta_{0}\right)\right)$.

Corollary 1.6. In Theorem 1.5, if $f(z) \in \mathcal{O}(\Omega)$ and $\theta_{0}>\pi /(2 \gamma)+\pi$, then $u(z)$ $\in \mathcal{O}(\Omega)$.

Corollary follows from Proposition 2.9.

Theorem 1.7. Suppose that $L\left(z, \partial_{z}\right)$ satisfies the conditions (1.11)-(a), (b), (c). Let $\theta_{0}$ be an arbitrary positive constant and $u(z) \in \mathcal{O}\left(\Omega\left(\theta_{0}\right)\right)$ be a solution of

$$
L\left(z, \partial_{z}\right) u(z)=f(z) \in \tilde{\pi}-A s y_{(\kappa)}\left(\Omega\left(\theta_{0}\right)\right),
$$

where $0<\kappa \leqq \gamma=\sigma_{p-1}-1$. If $u(z) \in \mathcal{O}_{(\gamma)}\left(\Omega\left(\theta_{0}\right)\right)$, then $u(z) \in \mathscr{M}-A s y_{(k)}\left(\Omega\left(\theta_{0}\right)\right)$.

Corollary 1.8. In Theorem 1.7, if $f(z) \in \tilde{\mathscr{M}}(\Omega-K)$ and $\theta_{0}>\pi /(2 \gamma)+2 \pi$, then $u(z) \in \tilde{\pi}(\Omega-K)$.

Corollary 1.8 also follows from Proposition 2.9.

Remark 1.9. Suppose that $L\left(z, \partial_{z}\right)$ satisfies (1.11) then $d_{k_{i}}=s_{k_{i}}=l_{k_{i}}$ and 
$j\left(k_{i}, l_{k_{i}}\right)=0$ for $0 \leqq i \leqq p-1$. The condition (1.11)-(a) means that $K=\left\{z_{0}=0\right\}$ is a irregular characteristic surface defined in [9], and the conditions (1.11)-(b), (c) mean that the $z$-th localization of $L\left(z, \partial_{z}\right)$ on $K$ is $a_{k_{i}, s_{k_{i}}}\left(0, z^{\prime}, \partial^{\prime}\right)$ and the $(p-1)$-th localization on $K$ is a function $a_{k_{p-1}, 0}\left(0, z^{\prime}\right)$. It follows from (1.5) that there are $r \geqq 0$ and $\hat{\xi}^{\prime} \neq 0$ such that

$$
\prod_{i=0}^{p-1} a_{k_{i}, s_{k_{i}}}\left(0, z^{\prime}, \hat{\xi}^{\prime}\right) \neq 0 \text { for }\left|z^{\prime}\right|=r .
$$

Many of the results in this paper were announced in [11], where (1.11)(a), (b), and instead of (1.11)-(c), only $d_{m}=s_{m}$ are assumed. The author thinks that the assertions in Theorem 1.5 and others hold under these weaker conditions, but they can not be shown by the method in this paper.

We give other results in the present paper which are used to prove the preceding Theorems. In the following the coordinate $(w, z, t)$ means a point in $C^{1} \times C^{n+1} \times C^{N+1}$. Put

$$
W_{\delta}=\left\{(w, z, t) ;|w|>(\sin \delta)\left|t_{0}\right|,|z| \leqq r_{1},\left|t_{0}\right| \leqq r_{3}, r_{2} \leqq\left|t_{i}\right| \leqq r_{3} \quad(i \geqq 1)\right\},
$$

where $0 \leqq \delta<\pi / 2$ and $0<r_{1}<r_{2}<r_{3}$.

Definition 1.10. $K\left(W_{\delta}\right)$ is the set of all $K(w, z, t) \in \mathcal{O}\left(\widetilde{W}_{\delta}\right)$ such that: for any fixed $w, K(w, z, t)$ is single valued holomorphic in $(z, t)$, and for any fixed $(z, t)$ it is holomorphic on the universal covering space of $\left\{w \in C^{1} ;(\sin \delta)\left|t_{0}\right|<\right.$ $|w|<+\infty\}$.

Now let us define integral operators, using $K(w, z, t) \in K\left(W_{\delta}\right)$. Put $\Omega=$ $\left\{t \in C^{N+1} ;|t| \leqq r_{3}\right\}$ and $U=\left\{z \in C^{n+1} ;|z| \leqq r_{1}\right\}$. Firstly we define a path $T(a, b)$ in $t$-space, $0<b-a \leqq 2 \pi, T(a, b)=T_{0}(a, b) \times T^{\prime} \subset C^{1} \times C^{N} . \quad T_{0}(a, b)=T_{0}^{1}(a, b)+$ $T_{0}^{2}(a, b)+T_{0}^{3}(a, b)$ is a path in $t_{0}$-space, where $T_{0}^{1}(a, b)=\left\{t_{0}=\left((1-s) r_{3}+s \eta\right) e^{i a}\right.$; $0 \leqq s \leqq 1\}, T_{0}^{2}(a, b)=\left\{t_{0}=\eta e^{i \varphi} ; a \leqq \varphi \leqq b\right\}$ and $T_{0}^{3}(a, b)=\left\{t_{0}=\left(s r_{3}+(1-s) \eta\right) e^{i b} ; 0 \leqq\right.$ $s \leqq 1\} \quad\left(0<\eta<r_{3}\right)$, (see Fig. 1.2.). $\quad T^{\prime}$ is the product of paths $\left\{t_{i}=r_{3} e^{i \varphi} ; 0 \leqq \varphi \leqq 2 \pi\right\}$ $(i=1,2, \cdots, N)$ in $C^{N}$. In the later sections we use the path $T$ when $N=n$ or $N=n-1$. When $N=n-1$, we use the notation $t=\left(t_{0}, t_{2}, \cdots, t_{n}\right)=\left(t_{0}, t^{\prime \prime}\right)$ and $T^{\prime}$ is denoted by $T^{\prime \prime}$.

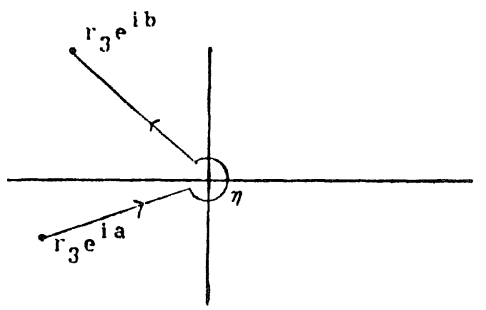

Fig。 1.2

Let us define for $f(z) \in \mathcal{O}(\Omega(a, b)), b-a>2 \delta$, 


$$
(K f)(z)=\int_{T} K\left(t_{0}-z_{0}, z, t\right) f(t) d t
$$

where $T=T_{0}\left(a^{\prime}, b^{\prime}\right)$ with $a<a^{\prime}<b^{\prime}<b$ and $2 \delta<b^{\prime}-a^{\prime} \leqq 2 \pi$. We will show in $\S 5$ that if $f(z) \in \mathcal{O}(\Omega(a, b)), b-a>2 \delta$, then $(K f)(z) \in \mathcal{O}(U(a+\delta, b-\delta))$ for a neighbourhood $U$ of $z=0$.

In order to show the preceding theorems we need the following Theorems 1.11 and 1.13 .

Theorem 1.11. Suppose that $L\left(z, \partial_{z}\right)$ satisfies the conditions
(a) $\sigma_{1}>1$,
(b) $d_{k_{p-1}}=0$,
(c) $a_{k_{p-1}, 0}(0) \neq 0$.

Put $\gamma=\sigma_{p-1}-1$ and $\alpha=\left(\sigma_{p-1}-1\right) / \sigma_{p-1}$. For given $\phi \in \boldsymbol{R}$ and any small $\delta_{0}>0$, there are $G(\psi ; w, z, t)=G_{\delta_{0}}(\psi ; w, z, t) \in K\left(W_{\delta_{0}}\right)$ and $G_{R}(\psi ; w, z, t)=G_{R, \delta_{0}}(\psi ; w, z, t)$ $\in K\left(W_{\hat{o}_{0}}\right)$ with the following (1)-(5). Let $f(z) \in \mathcal{O}(\Omega(a, b)), b-a>2 \delta_{0}$, and $\left(G^{\psi} f\right)(z)$ and $\left(G_{R}^{\dot{\omega}} f\right)(z)$ be operators defined by (1.16).

(1) There is a neighbourhood $U$ of $z=0$ such that $\left.\left(G^{\dot{\psi}} f\right)(z)\right),\left(G_{R}^{\omega} f\right)(z) \in$ $\mathcal{O}\left(U\left(a+\delta_{0}, b-\delta_{0}\right)\right)$ and

$$
L\left(z, \partial_{z}\right)\left(G^{\psi} f\right)(z)=f(z)+\left(G_{R}^{\psi} f\right)(z)+a \text { holomorphic function on } U .
$$

(2) Let $f(z) \in \mathcal{O}_{(\gamma), h}(\Omega(a, b))$, where $\phi-\pi /(2 \alpha)+\pi / 2<a<b<\phi+\pi /(2 \alpha)+3 \pi / 2$ and $b-a>\pi+2 \delta_{0}$. Then for any $\varepsilon>\delta_{0}$ there is a constant $c=c(\varepsilon)>0$ such that $\left(G^{\prime} f\right)(z) \in \mathcal{O}_{(\gamma), c h(}(U(a+\varepsilon, b-\varepsilon))$.

(3) Suppose $\psi^{\prime}<\psi^{\prime \prime}$ and $0<\alpha \delta_{0}<\left(\pi-\alpha\left|\psi^{\prime \prime}-\psi^{\prime}\right|\right) / 2$. Let $f(z) \in \mathcal{O}_{(r), h}(\Omega(a, b))$, where $\phi^{\prime \prime}-(\pi / 2 \alpha)+\pi / 2<a<b<\psi^{\prime}+\pi /(2 \alpha)+3 \pi / 2$ and $b-a>\pi+2 \delta_{0}$. Then there is an $h_{0}$ such that if $0<h<h_{0},\left(G^{\dot{b}^{\prime}} f\right)(z)-\left(G^{\dot{\phi}^{\prime \prime}} f\right)(z) \in A s y_{(\gamma)}\left(U\left(a+\delta_{0}, b-\delta_{0}\right)\right)$.

(4) Let $\kappa>0$ be arbitary. Let $f(z) \in A s y_{(\kappa)}(\Omega(a, b))$, where $\phi<a<b<\phi+2 \pi$ and $b-a>2 \delta_{0}$. Then $\left(G^{\psi} f\right)(z) \in A s y_{(\kappa)}\left(U\left(a+\delta_{0}, b-\delta_{0}\right)\right)$.

(5) Let $f(z) \in \mathcal{O}_{(r), h}(\Omega(a, b))$. Suppose that one of the following conditions holds :

(i) $\phi-\pi /(2 \alpha)+\pi / 2<a<b<\phi+\pi /(2 \alpha)+3 \pi / 2$ and $b-a>\pi+2 \delta_{0}$,

(ii) $\psi-\pi /(2 \alpha)+3 \pi / 2<a<b<\psi+\pi /(2 \alpha)+\pi / 2$ and $b-a>2 \delta_{0}$.

Then there is an $h_{1}$ such that, if $0<h<h_{1},\left(G_{R}^{\psi} f\right)(z) \in A s y_{(\gamma)}\left(U\left(a+\delta_{0}, b-\delta_{0}\right)\right)$.

Remark 1.12. Suppose $L\left(z, \partial_{z}\right)$ satisfies the conditions (1.11)-(a), (b), which are the same as (1.17)-(a), (b). Then it is written in the form

$$
L\left(z, \partial_{z}\right)=a_{k_{p-1}, 0}(z)\left(\partial_{0}\right)^{k p-1}+\sum_{(k, l) \neq\left(k_{p-1}, 0\right)} z_{0}^{j(k, l)} a_{k, l}\left(z, \partial^{\prime}\right)\left(\partial_{0}\right)^{k-l},
$$

where $k-d_{k, l}=k-(l+j(k, l))<k_{p-1}$ for $(k, l) \neq\left(k_{p-1}, 0\right)$. So, if $(1.17)-(\mathrm{c})$ is valid, then for a given formal power series $f(z)$ of $z_{0}, f(z)=\sum_{n=0}^{\infty} f_{n}\left(z^{\prime}\right)\left(z_{0}\right)^{n} / n !$, $f_{n}\left(z^{\prime}\right) \in \mathcal{O}\left(\Omega^{\prime}\right)$, there is a formal power series $u(z)=\sum_{n=k}^{\infty}{ }_{p-1} u_{n}\left(z^{\prime}\right)\left(z_{0}\right)^{n} / n !, u_{n}\left(z^{\prime}\right)$ $\in \mathcal{O}\left(\Omega^{\prime}\right)$, such that $L\left(z, \partial_{z}\right) u(z)=f(z)$ as a formal power series of $z_{0}$. 
We have the following which we apply to the proof of Theorem 1.7.

Theorem 1.13. Suppose that $L\left(z, \partial_{z}\right)$ satisfies the condition (1.17) and put $\gamma=\sigma_{p-1}-1$. Let $f(z) \in A s y_{(\kappa)}\left(\Omega\left(\theta_{0}\right)\right)$ with $0<\theta_{0} \leqq \pi / 2 \kappa$ and $0<\kappa \leqq \gamma$. Then there is a $\tilde{v}(z) \in \tilde{M}-A s y_{(\kappa)}\left(U\left(\theta_{0}\right)\right)$ such that $L\left(z, \partial_{z}\right) \tilde{v}(z)-f(z) \log z_{0} \in A s y_{(\kappa)}\left(U\left(\theta_{0}\right)\right)$, where $U$ is a neighbourhood of $z=0$.

At the end of this section we show an example. Let $L\left(z, \partial_{z}\right)$ be an operator of the form

$$
L\left(z, \partial_{z}\right)=\left(\partial_{0}\right)^{k}+A_{l}\left(z, \partial^{\prime}\right)\left(\partial_{0}\right)^{m_{1}-l}+A_{m_{2}}\left(z, \partial^{\prime}\right),
$$

where ord. $A_{l}\left(z, \partial^{\prime}\right)=l \geqq 1$, and ord. $A_{m_{2}}\left(z, \partial^{\prime}\right)=m_{2}$. We assume that $k<m_{1}<m_{2}$, (P.S. $\left.A_{l}\right)\left.\left(z, \xi^{\prime}\right)\right|_{z_{0}=0} \neq 0$ and (P.S. $\left.A_{m_{2}}\right)\left.\left(z, \xi^{\prime}\right)\right|_{z_{0}=0} \neq 0$, where (P.S. $\left.A\right)(z, \xi)$ means the principal symbol of $A\left(z, \partial_{z}\right)$.

Case (1) $\quad\left(m_{2}-l\right) /\left(m_{2}-m_{1}\right) \leqq m_{2} /\left(m_{2}-k\right)$. We have $\sigma_{1}=m_{2} /\left(m_{2}-k\right)>1, \sigma_{2}=1$, $p=2$ and $\gamma=\sigma_{1}-1$. The conditions (1.11)-(a), (b), (c) are satisfied.

Case $(2) \quad\left(m_{2}-l\right) /\left(m_{2}-m_{1}\right)>l /\left(m_{1}-k\right)>1$. We have $\sigma_{1}=\left(m_{2}-l\right) /\left(m_{2}-m_{1}\right), \sigma_{2}=$ $l /\left(m_{1}-k\right), \quad \sigma_{3}=1, \quad p=3$ and $\gamma=\sigma_{2}-1$. The conditions (1.11)-(a), (b), (c) are satisfied.

Case (3) $\quad\left(m_{2}-l\right) /\left(m_{2}-m_{1}\right)>1 \geqq l /\left(m_{1}-k\right)$. We have $\sigma_{1}=\left(m_{2}-l\right) /\left(m_{2}-m_{1}\right), \sigma_{2}$ $=1, p=2, d_{k_{1}}=l \geqq 1$. So (1.11)-(b) does not hold.

Case (4) $1 \geqq\left(m_{2}-l\right) /\left(m_{2}-m_{1}\right) \geqq l /\left(m_{1}-k\right)$. We have $\sigma_{1}=1$ and $d_{k_{0}}=m_{2}$. Neither (1.11)-(a) nor (b) is valid.

According to the classification of characteristic surfaces in [9], in Cases (1)-(3) $K=\left\{z_{0}=0\right\}$ is irregular characteristic and in Case (4) $K=\left\{z_{0}=0\right\}$ is regular characteristic.

\section{§2. Function Spaces}

In $\S 1$ we introduced some classes of holomorphic functions. In the present section we give some properties of them, which are used to show Theorems and Corollaries stated in $\S 1$. Some of them were given in [12] and we refer the proofs of them to it. In the definitions of function spaces in $\S 1, z$ means the $(n+1)$-variables, $z=\left(z_{0}, z^{\prime}\right) \in C^{n+1}$, but the variable $z_{0}$ is important and other variables $z^{\prime}$ are not. Hence $z$ or $t$ means one complex variable in $\S 2$ except Proposition 2.8.

Firstly we study functions with asymptotic expansions on the half axis. Let $u(z)$ be a function defined on the line $\{z ; \arg z=\varphi, 0 \leqq|z|<A\}$ having the $\kappa$-asymptotic expansion,

$$
u(z) \sim \sum_{k=0}^{+\infty} c_{k} z^{k},
$$

that is, if for any $N \geqq 1$ 


$$
\left|u(z)-\sum_{k=0}^{N-1} c_{k} z^{k}\right| \leqq A R^{-N} \Gamma(N / \kappa+1) \mid z_{1}^{\prime N}
$$

We may assume $\varphi=0$. We have from (2.2)

$$
\left|c_{k}\right| \leqq A R^{-k} \Gamma(k / \kappa+1) .
$$

Conversely, let $\left\{c_{k}\right\}(k=0,1, \cdots)$ be a sequence satisfying (2.3). Put

$$
g(t)=\sum_{k=0}^{+\infty} \frac{c_{k} t^{k}}{\Gamma(k / \kappa+1)} \quad\left(t \in C^{1}\right),
$$

which is holomorphic in $\left\{t \in C^{1} ;|t|<R\right\}$ and $|g(t)| \leqq A(1-|t / R|)^{-1}$. Put

$$
v(z)=z^{-\kappa} \int_{0}^{c} \exp \left(-z^{-\kappa} t\right) g\left(t^{1 / \kappa}\right) d t, \quad 0<c^{1 / \kappa}<R,
$$

which depends on $c$. Then we have

Proposition 2.1. (1). $v(z) \in \mathcal{O}\left(\widetilde{C^{1}-\{0\}}\right)$.

(2). $v(z)$ has the $\kappa$-asymptotic expansion as $z \rightarrow 0$ in $\{z ;|\arg z|<\pi / 2 \kappa\}$, that is, there is an $A(c)$ such that for any $N$

$$
\left|v(z)-\sum_{k=0}^{N-1} c_{k} z^{k}\right| \leqq A(c) c^{-N / k}\left(\cos \left(\kappa \theta^{\prime}\right)\right)^{-N / \Lambda-1} \Gamma(N / \kappa+1)|z|^{N}
$$

holds in $\left\{z ;|\arg z|<\theta^{\prime}\right\}$ for any $\theta^{\prime}$ with $0<\theta^{\prime}<\pi / 2 \kappa$.

Set $w(z)=u(z)-v(z)$. By Proposition 2.1, $u(z)$ and $v(z)$ have the same asymptotic expansion on the positive real axis as $z \rightarrow+0$. Hence $w(z) \sim 0$ as $z \rightarrow 0$ on the positive real axis. More precisely we have

Lemma 2.2. $|w(x)| \leqq A c^{-n / \kappa} \Gamma(n / \kappa+1)|x|^{n}$ for each $n$, and $|u(x)| \leqq$ $C\left(c x^{-n}\right)^{1 / 2} \exp \left(-c x^{-\kappa}\right)$ for $0 \leqq x \leqq 1$, where $A$ and $C$ depend on $c$.

The proofs of Proposition 2.1 and Lemma 2.2 were in [12]. We'll define the $\kappa$-Laplace transform and investigate relations between functions with the $\kappa$-asymptic expansion and their $\kappa$-Laplace transforms. Let $\chi(x)$ be a continuous function on $(0, A)(A>0)$ with $|\chi(x)| \leqq C \exp \left(h|x|^{-1}\right)(\kappa>0)$. We define the $\kappa$ Laplace transform $\hat{\chi}(\xi)$ of $\chi(x)$ by

$$
\hat{\chi}(\xi)=\int_{a}^{+\infty} \exp (\xi x) \chi\left(x^{-1 / \kappa}\right) x^{-1} d x \quad\left(a>A^{-\kappa}\right),
$$

which is holomorphic in $\{\xi ; \operatorname{Re} \xi<-h\} . \hat{\chi}(\xi)$ depends on $a$, we may choose any $a$ with $a>A^{-\kappa}$ and fix it. The inversion formula is given by

$$
\chi(x)=\frac{x^{-\kappa}}{2 \pi i} \int_{d-i \infty}^{d+i \infty} \exp \left(-\xi x^{-\kappa}\right) \hat{\chi}(\xi) d \xi \quad(d<-h) \quad \text { for } 0<x<a^{-1 / \kappa}
$$

Let $\hat{\imath}(\xi)$ be the $\kappa$-Laplace transform of $u(x)$ with the $\kappa$-asymptotic expansion (2.1). Since $\hat{u}(\xi)=\hat{v}(\xi)+\hat{\imath}(\xi), v(z)$ being defined by (2.5), we study $\hat{\imath}(\xi)$ and 
$\hat{w}(\xi)$. We have

Lemma 2.3. (1) $\hat{w}(\xi) \in \mathcal{O}(\{\xi ; \operatorname{Re} \xi<c\})$.

(2) $\hat{v}(\xi) \in \mathcal{O}\left(\left\{\xi ; \xi \in C^{1}-[0, c]\right\}\right)$. It has the holomorphic prolongation around $\xi=0$ so that $\left.\hat{v}(\xi) \in \mathcal{O}\left(\tilde{\Xi}_{0}\right), \Xi_{0}=\left\{\xi \in C^{1} ; 0<|\xi|<c\right\}\right)$.

(3) $|\hat{v}(\xi)| \leqq M_{r, \theta}|\log \xi|$ in $\{\xi ;|\arg \xi|<\theta, 0<|\xi|<r\}$ for any $\theta$ and $0<r<c$.

(4) $\left\{\hat{v}(\xi)-\hat{v}\left(\xi e^{2 \pi i}\right)\right\} / 2 \pi i=g\left(\xi^{1 / \kappa}\right)$, where $g(t)$ is defined by (2.4) and $\xi^{1 / \kappa}=$ $|\xi|^{1 / \kappa} e^{i(\arg \xi) / \kappa}$.

It follows from Lemma 2.2 that $\hat{w}(\xi) \in \mathcal{O}(\{\xi ; \operatorname{Re} \xi<c\})$. We refer the proof of (2)-(4) to [12]. Thus we have

Proposition 2.4. Let $u(x)$ be a function with the asymptotic expansion (2.1) on $[0, A)$. Then there is a constant $c>0$ such that

(1) $\hat{u}(\xi)$ is holomorphic in $\{\xi ; \operatorname{Re} \xi<c, \xi \notin[0, c)\}$,

(2) $\hat{u}(\xi)$ is holomorphically extensible onto $\Xi_{0}=\{\xi ; 0<|\xi|<c\}$ such that $\hat{u}(\xi)$ $\in \mathcal{O}\left(\tilde{\Xi}_{0}\right)$,

$$
|\hat{u}(\xi)| \leqq M_{r, \theta}|\log \xi| \quad \text { in }\{\xi ;|\arg \xi|<\theta, 0<|\xi|<r\} \quad(0<r<c)
$$

for any $\theta>0$ and $\left\{\hat{u}(\xi)-\hat{u}\left(\xi e^{2 \pi i}\right)\right\} / 2 \pi i=g\left(\xi^{1 / r}\right)$.

Next we consider functions holomorphic in a sector $\Omega(a, b), \Omega=\{z ;|z|<R\}$. In the sequel we only consider $u(z) \in \mathcal{O}_{(\kappa), h}(\Omega(a, b))$. We can define the $\kappa$ Laplace transform $\hat{u}(\xi)$ of $u(z)$ by

$$
\hat{u}(\xi)=\int_{\Lambda}^{+\infty e^{i \varphi}} \exp (\xi z) u\left(z^{-1 / \kappa}\right) z^{-1} d z \quad(a<-\varphi / \kappa<b),
$$

where $|A|>R^{-\kappa}$ and $a<-(\arg A) / \kappa<b, \hat{u}(\xi)$ depends on $A$. We may choose any $A$ satisfying the conditions and we fix it. $\hat{u}(\xi)$ is holomorphic on the set

$$
\begin{aligned}
& \Xi(h, a, b) \\
& =\bigcup_{a<-\varphi / k<b}\left\{\xi=|\xi| e^{i \psi} \in \overparen{C^{1}-\{0\}} ;|\xi| \cos (\phi+\varphi)<-h,|\psi+\varphi-\pi|<\pi / 2\right\} .
\end{aligned}
$$

We have

Theorem 2.5. Let $u(z) \in \mathcal{O}_{(\kappa), h}(\Omega(a, b))$. Suppose that the $\kappa$-Laplace transform $\hat{u}(\xi) \in \mathcal{O}(\boldsymbol{\Xi}(h, a, b))$ is holomorphically prolonged to the punctured disk $\tilde{\Xi}_{0}, \boldsymbol{\Xi}_{0}$ $=\{0<|\xi|<c\}$, for some $c>h$ so that

(i) for any $\Phi>0|\hat{u}(\xi)| \leqq M_{\Phi}|\log \xi|$ in $\left\{\xi \in \tilde{\Xi}_{0} ;|\arg \xi-\pi|<\Phi\right\}$, and

(ii) $F(\xi)=\left\{\hat{u}(\xi)-\hat{u}\left(\xi e^{2 \pi i}\right)\right\} / 2 \pi i$ is a convergent power series of $\xi^{1 / \pi}$ at $\xi=0$,

$$
F(\xi)=\sum_{k=0}^{+\infty} c_{K} \xi^{k / \kappa} / \Gamma(N / \kappa+1) .
$$

Then there is an $h_{0}=h_{0}(c)>0$ such that the following hold. If $h<h_{0}$, there are $a^{\prime}=a^{\prime}(h)$ and $b^{\prime}=b^{\prime}(h){ }_{\mathrm{s}}^{\mathrm{s}}\left(a<a^{\prime}<b^{\prime}<b\right)$ such that $u(z)$ has the $\kappa$-asymptotic 
expansion (2.1) in $\Omega\left(a^{\prime}, b^{\prime}\right)$ and $\lim _{h \rightarrow+0} a^{\prime}(h)=a$ and $\lim _{h \rightarrow+0} b^{\prime}(h)=b$.

Proof. We may assume that $a=-\theta_{0}$ and $b=\theta_{0}\left(\theta_{0}>0\right)$. Firstly we note that there exists $0<h_{0}<c$ such that if $0<h<h_{0}, \Xi\left(h,-\theta_{0}, \theta_{0}\right) \cup \tilde{\Xi}_{0} \supset\left\{\xi=|\xi| e^{i \psi}\right.$; $\left.\xi \neq 0,|\psi-\pi|<\theta^{\prime \prime}\right\}$ for some $\theta^{\prime \prime}=\theta^{\prime \prime}(h)>\pi / 2$ with $\lim _{h \rightarrow+0} \theta^{\prime \prime}(h)=\kappa \theta_{0}+\pi / 2$. By the deformation of the integration path to the right half plane, the inverse $\kappa$ Laplace transform is given by

$$
u(z)=\frac{z^{-\kappa}}{2 \pi i}\left(\int_{\infty i e}^{d i\left(\pi+\theta^{\prime}\right)}+\int_{d}^{\infty e^{i\left(\pi-\theta^{\prime}\right)}}\right) \exp \left(-\xi z^{-\kappa}\right) \hat{u}(\xi) d \xi
$$

for $z \in \Omega\left(\left(\theta^{\prime}-\pi / 2\right) / \kappa\right)\left(\pi / 2<\theta^{\prime} \leqq \pi\right)$. From the assumption, $\hat{u}(\xi)$ has at most the logarithmic growth at $\xi=0$. Hence, by deforming the integration path (see Fig. 2.1), we have for $z \in \Omega\left(\left(\theta^{\prime}-\pi / 2\right) / \kappa\right)$ with $\pi / 2<\theta^{\prime}<\theta^{\prime \prime}$ and $\theta^{\prime} \leqq \pi$,

$$
\begin{aligned}
u(z) & =\frac{z^{-\kappa}}{2 \pi i}\left(\int_{c c^{2 \pi i}}^{0}+\int_{0}^{c}\right) \exp \left(-\xi z^{-\kappa}\right) \hat{u}(\xi) d \xi+s_{\theta^{\prime}}(z) \\
& =z^{-\kappa} \int_{0}^{c} \exp \left(-\xi z^{-\kappa}\right) F(\xi) d \xi+s_{\theta^{\prime}}(z),
\end{aligned}
$$

Here $F(\xi)=\hat{u}(\xi)-\hat{u}\left(\xi e^{2 \pi i}\right)$ and

$$
s_{\theta^{\prime}}(z)=\frac{z^{-\kappa}}{2 \pi i}\left(\int_{\infty e^{i\left(\pi+\theta^{\prime}\right)}}^{c e^{2 \pi \imath}}+\int_{c}^{\infty e^{i\left(\pi-\theta^{\prime}\right)}}\right) \exp \left(-\xi z^{-\kappa}\right) \hat{u}(\xi) d \xi .
$$

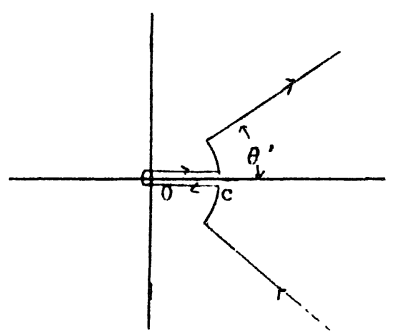

Fig. 2.1

For $z$ with $|\arg z|<\pi / 2 \kappa$ we have the $\kappa$-asymptotic expansion

$$
z^{-\kappa} \int_{0}^{c} \exp \left(-\xi z^{-\kappa}\right) F(\xi) d \xi \sim \sum_{k=0}^{+\infty} c_{k} z^{k}
$$

and

$$
\left|s_{\theta^{\prime}}(z)\right| \leqq A_{\theta^{\prime}, \hat{\delta}} \exp \left(-\left.c_{\delta} ! z\right|^{-\kappa}\right), c_{\dot{\delta}}>0,
$$

in $\left\{z ; 0<|z|<A^{\prime}, \pi / 2-\theta^{\prime}+\delta<\kappa\right.$ arg $\left.z<-\pi / 2+\theta^{\prime}-\delta\right\}$ for any $\delta>0$. Hence, we have the $\kappa$-asymptotic expansion of $u(z)$

$$
u(z) \sim \sum_{k=0}^{+\infty} c_{k} z^{k} \quad \text { in } \Omega\left(\left(\theta^{\prime}-\pi / 2\right) / \kappa\right) .
$$

Moreover if follows from the rotation of $z$ and using the above method again 
that $u(z)$ has the $\kappa$-asymptotic expansion (2.18) in $\Omega\left(\left(\theta^{\prime \prime}-\pi / 2\right) / \kappa\right)$. Put $-a^{\prime}(h)$ $=b^{\prime}(h)=\left(\theta^{\prime \prime}-\pi / 2\right) / \kappa$ and as mentioned above $\lim _{h \rightarrow+0} \theta^{\prime \prime}(h)=\kappa \theta_{0}+\pi / 2$. Hence $\lim _{h \rightarrow+0}$ $-a^{\prime}(h)=\lim _{h \rightarrow+0} b^{\prime}(h)=\left(\theta^{\prime \prime}-\pi / 2\right) / \kappa=\theta_{0}$.

From Proposition 2.4 and Theorem 2.5 we have

Corollary 2.6. Let $u(z) \in \mathcal{O}_{(x)}(\Omega(a, b))$. Then $u(z)$ has the $\kappa$-asymptotic expansion in $\Omega(a, b)$, if and only if the $\kappa$-Laplace transform $\hat{\imath}(\xi) \in \mathcal{O}\{\xi ; \kappa a-\pi / 2<$ $\arg \xi-\pi<\kappa b+\pi / 2\}$ satisfies the following (1) and (2).

(1) $\hat{u}(\xi)$ has holomorphic extension onto $\Xi_{0}=\{\xi ; 0<|\xi|<c\}$ such that $\hat{u}(\xi) \in$ $\mathcal{O}\left(\tilde{\Xi_{0}}\right)$ and $|\hat{\imath}(\xi)| \leqq M_{\hat{o}}|\log \xi|$ on $\left\{\xi \in \tilde{\Xi}_{0} ; \kappa a-\pi / 2+\delta<\arg \xi-\pi<\kappa b+\pi / 2-\delta\right\}$ for any $\delta>0$.

(2) $F(\xi)=\left\{\hat{\imath}(\xi)-\hat{\imath}\left(\xi e^{2 \pi i}\right)\right\} /(2 \pi i)$ has the convergent power series of $\xi^{1 / \hbar}$ at $\xi=0$ such as (2.12).

Remark 2.7. Put

$$
\hat{U}_{k}(\xi)=\frac{1}{2 \pi i} \int_{0}^{r} \frac{t^{k / k}}{t-\xi} d t .
$$

Then $\hat{U}_{k}(\xi)-\hat{U}_{k}\left(\xi e^{2 \pi i}\right)=\xi^{k / \kappa}$ for $|\xi|<r$. Define

$$
\hat{U}(\xi)=\sum_{k=0}^{+\infty} c_{k} \hat{U}_{k}(\xi) / \Gamma(N / \kappa+1) .
$$

Then $\hat{U}(\xi)-\hat{U}\left(\xi e^{2 \pi i}\right)=\sum_{k=0}^{+\infty} c_{k} \xi^{k / \kappa} / \Gamma(N / \kappa+1)$ and it is easy to show that $\hat{U}(\xi)$ has at most logarithmic growth at $\xi=0$. Suppose that conditions (1) and (2) in Corollary 2.6 hold. We have $\hat{u}(\xi)-\hat{U}(\xi)=\hat{u}\left(\xi e^{2 \pi i}\right)-\hat{U}\left(\xi e^{2 \pi i}\right)$ and $\hat{u}(\xi)-\hat{U}(\xi) \in$ $\mathcal{O}\{(0<|\xi|<c\})$. Since $\hat{u}(\xi)$ and $\hat{U}(\xi)$ has at most logarithmic growth at $\xi=0$, $\hat{\imath}(\xi)-\hat{U}(\xi) \in \mathcal{O}(|\xi|<c)$. Therefore the behaviour of $\hat{u}(\xi)$ at $\xi=0$ is characterized by $\hat{U}(\xi)$.

In the next proposition $z=\left(z_{0}, z^{\prime}\right) \in C^{n+1}$ and $\Omega=\Omega_{0} \times \Omega^{\prime}$ is a polydisk with the center $z=0$ in $C^{n+1}$.

Proposition 2.8. Let $u(z) \in \mathcal{O}_{(x)}(\Omega(a, b))$ and $D$ be a non empty open set in $\Omega^{\prime}$. Suppose that $u(z)$ has the $\kappa$-asymptotic expansion with respect to $z_{0}$ as $z_{0} \rightarrow 0$ on $\Omega_{0}(a, b) \times D$, then $u(z)$ has also the $\kappa$-asymptotic expansion in $\Omega(a, b)$.

Proof. We may assume $-a=b=\theta_{0}$. We have $\hat{u}\left(\xi, z^{\prime}\right) \in \mathcal{O}\left(Z_{\kappa \theta_{0}+\pi / 2} \times \Omega^{\prime}\right)$, where $Z_{\theta}=\left\{\xi \in \widetilde{C^{1}-\{0\}} ;|\arg \xi-\pi|<\theta\right\}$. If $z^{\prime} \in D, \hat{u}\left(\xi, z^{\prime}\right) \in \mathcal{O}\left(Z_{+\infty} \times D^{\prime}\right)$. Hence, it follows from the theory of the extension of holomorphic functions of several complex variables that $\hat{u}\left(\xi, z^{\prime}\right)$ is holomorphically extensible around $\xi=0$ for $z^{\prime} \in \Omega^{\prime}$, that is, $\hat{u}\left(\xi, z^{\prime}\right) \in \mathcal{O}\left(Z^{*}\right), Z^{*}=\left\{\left(\xi, z^{\prime}\right) \in \widehat{C^{1}-\{0\}} \times \Omega^{\prime} ; 0<|\xi|<c\left(\left|z^{\prime}\right|\right)\right\}$ for some $c\left(\left|z^{\prime}\right|\right)>0$ (see [4]). We have $F\left(\xi, z^{\prime}\right)=\left\{\hat{u}\left(\xi, z^{\prime}\right)-\hat{\imath}\left(\xi e^{2 \pi i}, z^{\prime}\right)\right\} /(2 \pi i)=$ 
$\sum_{k=0}^{+\infty} c_{k}\left(z^{\prime}\right) \xi^{k / \kappa} / \Gamma(k / \kappa+1)$, and $\hat{u}\left(\xi, z^{\prime}\right)-\hat{U}\left(\xi, z^{\prime}\right) \in \mathcal{O}\left(|\xi|<c\left(\left|z^{\prime}\right|\right)\right)$ as a function of $\xi$, where $\hat{U}\left(\xi, z^{\prime}\right)=\sum_{k=0}^{+\infty} c_{k}\left(z^{\prime}\right) \hat{U}_{k}(\xi) / \Gamma(k / \kappa+1)$ is defined by $(2.20)$ for $\hat{u}\left(\xi, z^{\prime}\right)$. Hence it follows from Remark 2.7 that the conditions (1) and (2) in Corollary 2.6 hold. So $u(z)$ has the $\kappa$-asymptotic expansion with respect to $z_{0}$ in $\Omega(a, b)$.

Proposition 2.9. (1) Let $f(z) \in A s y_{(\kappa)}(\Omega(a, b))$. If $b-a>\pi / \kappa+2 \pi$, then $f(z) \in \mathcal{O}(\Omega)$.

(2) Let $f(z) \in \tilde{M}-A s y_{\{\kappa\}}(\Omega(a, b))$. If $b-a>\pi / \kappa+4 \pi$, then $f(z) \in \tilde{M}(\Omega-K)$.

Proof. (1) Put $F(z)=f(z)-f\left(z e^{2 \pi i}\right), z \in \Omega(a, b-2 \pi)$. Then $F(z) \sim 0$. More precisely for any $N \geqq 1$ we have

$$
|F(z)| \leqq A B^{N} \Gamma(N / \kappa+1)\left|z_{0}\right|^{N} \quad \text { in } \Omega\left(a^{\prime}, b^{\prime}-2 \pi\right)\left(a<a^{\prime}<b^{\prime}<b\right) .
$$

Choose $a^{\prime}$ and $b^{\prime}$ such that $b^{\prime}-a^{\prime}-2 \pi>\pi / \kappa$. From (2.21) we have $|F(z)| \leqq$ $A \exp \left(-c\left|z_{0}\right|^{-\kappa}\right)$ in $\Omega\left(a^{\prime}, b^{\prime}-2 \pi\right)$ for some $c>0$. Since $\left(b^{\prime}-2 \pi\right)-a^{\prime}>\pi / \kappa, F(z)$ $\equiv 0$. So $f(z)$ is single valued on $\Omega-K$ and bounded. Consequently $f(z)$ is holomorphic in $\Omega$.

(2) Put $F(z)=f(z)-f\left(z e^{2 \pi i}\right), z \in \Omega(a, b-2 \pi)$. Then $F(z) \in A s y_{(\kappa)}\left(\Omega\left(a^{\prime}, b^{\prime}\right.\right.$ $-2 \pi)$ ). We can choose $a^{\prime}$ and $b^{\prime}$ such that $\left(b^{\prime}-2 \pi\right)-a^{\prime}>\pi / \kappa+2 \pi$. Hence $F(z)$ $\in \mathcal{O}(\Omega)$ by (1). Put $g(z)=f(z)+(1 / 2 \pi i) F(z) \log z_{0}$. Then $g(z)-g\left(z e^{2 \pi i}\right)=F(z)-$ $1 /(2 \pi i)\left\{F(z)\left(\log z_{0}+2 \pi i\right)-F(z) \log z_{0}\right\}=0$. So $g(z)$ is single valued in $\Omega-K$. Since $|g(z)| \leqq A\left|z_{0}\right|^{-H}$ for some $H \geqq 0,\left\{z_{0}=0\right\}$ is at most a pole of $g(z)$. Thus $f(z)=g(z)-1 /(2 \pi i) F(z) \log z_{0} \in \tilde{\mathscr{N}}(\Omega-K)$.

As stated in $\S 1$, we have Corollary 1.6 (resp. 1.8) by Theorem 1.5 (resp. 1.7) and Proposition 2.9.

Proposition 2.10. Let $u(z) \in \mathcal{O}_{\{\kappa\}, h}(\Omega(a, b))$. Suppose that there exist $u_{\imath}(z)$ $\in \mathcal{O}_{\left(\kappa_{i}\right)}(\Omega(a, b))\left(i=1,2, \cdots, l, \kappa<\kappa_{1}<\kappa_{2}<\cdots<\kappa_{l}\right)$ such that $u(z)=\sum_{i=1}^{l} u_{i}(z)$ and each $u_{i}(z)$ has the $\kappa$-asymptotic expansion on $\left\{z ; \arg z=\varphi_{i},|z|<R\right\}\left(a<\varphi_{2}<b\right)$. Further assume that there is $a \varphi_{0} \in(a, b)$ such that $\left|\varphi_{i}-\varphi_{0}\right|<\pi / 2 \kappa_{\imath}$. Then there is an $h_{0}>0$ such that, if $0<h<h_{0}, u(z) \in A s y_{[\kappa]}\left(\Omega\left(a^{\prime}, b^{\prime}\right)\right)$ for some $a^{\prime}=a^{\prime}(h)$ and $b^{\prime}=b^{\prime}(h)\left(a<a^{\prime}<b^{\prime}<b\right)$ satisfying $\lim _{h \rightarrow+0} a^{\prime}(h)=a$ and $\lim _{h \rightarrow+0} b^{\prime}(h)=b$.

Proof. By the rotation of $z$, we may assume $a<0<b$ and $\varphi_{0}=0$. We have

$$
\hat{u}(\xi)=\int_{A}^{+\infty i \varphi} \exp (\xi z) u\left(z^{-1 / \kappa}\right) z^{-1} d z \quad\left(A>R^{-\kappa}, \arg z=\varphi\right)
$$

which is holomorphic in $\Xi(h, a, b)$ (see $(2.11)) . \hat{\imath}(\xi)$ is represented in the form

$$
\hat{u}(\xi)=\lim _{\varepsilon_{1} \rightarrow+0} \lim _{\varepsilon_{2} \rightarrow+0} \cdots \lim _{\varepsilon_{l} \rightarrow+0} \int_{a}^{+\infty e \imath \varphi} \exp \left(\xi z-\sum_{i=1}^{l} \varepsilon_{i} z^{\rho} i\right) u\left(z^{-1 / \kappa}\right) z^{-1} d z,
$$

where $\rho_{\imath}=\kappa_{i} / \kappa>1$ and $|\varphi|<\pi / 2 \rho_{l}$. Suppose $|\arg \xi-\pi|<\theta^{*}$, where $\theta^{*}=$ 
$\min \left\{\pi / 2-\left|\kappa \varphi_{i}\right| ; 1 \leqq i \leqq l\right\}>0$. Since $\left|\kappa \varphi_{l}\right|<\pi / 2 \rho_{l}$, by putting $\varphi=-\kappa \varphi_{l}$, we have

$$
\begin{aligned}
& \hat{u}(\xi)=\int_{A}^{+\infty e^{-i \kappa \varphi l}} \exp (\xi z) u\left(z^{-1 / \kappa}\right) z^{-1} d z \\
& =\lim _{\varepsilon_{1} \rightarrow+0} \lim _{\varepsilon_{2} \rightarrow+0} \cdots \lim _{\varepsilon_{l \rightarrow+0}} \int_{A}^{+\infty e^{-i \kappa \varphi} l} \exp \left(\xi z-\sum_{i=1}^{l} \varepsilon_{i} z^{\rho i}\right)\left(\sum_{i=1}^{l} u_{i}\left(z^{-1 / \kappa}\right)\right) z^{-1} d z, \\
& =\lim _{\varepsilon_{1} \rightarrow+0} \lim _{\varepsilon_{2} \rightarrow+0} \cdots \lim _{\varepsilon_{l-1 \rightarrow+0}} \int_{A}^{+\infty e^{-i \kappa \varphi_{l}}} \exp \left(\xi z-\sum_{i=1}^{l-1} \varepsilon_{i} z^{\rho_{\imath}}\right)\left(\sum_{i=1}^{l-1} u_{i}\left(z^{-1 / \kappa}\right)\right) z^{-1} d z \\
& +\lim _{\varepsilon_{1} \rightarrow+0} \lim _{\varepsilon_{2} \rightarrow+0} \ldots \lim _{\varepsilon_{l-1 \rightarrow+0}} \int_{\Lambda}^{+\infty e^{-i \kappa \varphi} l} \exp \left(\xi z-\sum_{i=1}^{l-1} \varepsilon_{i} z^{\rho i}\right) u_{l}\left(z^{-1 / \kappa}\right) z^{-1} d z \\
& =\lim _{\varepsilon_{1} \rightarrow+0} \lim _{\varepsilon_{2} \rightarrow+0} \cdots \lim _{\varepsilon_{l} \rightarrow-1 \rightarrow+0} \int_{A}^{+\infty e^{-i \kappa \varphi} l} \exp \left(\xi z-\sum_{i=1}^{l-1} \varepsilon_{i} z^{\rho_{i}}\right)\left(\sum_{i=1}^{l-1} u_{i}\left(z^{-1 / \kappa}\right)\right) z^{-1} d z \\
& \left.+\int_{A}^{+\infty e^{-i \kappa \varphi} l} \exp (\xi z)\right) u_{l}\left(z^{-1 / \kappa}\right) z^{-1} d z \text {. }
\end{aligned}
$$

Since $\left|\kappa \varphi_{l-1}\right|<\pi / 2 \rho_{l-1}$, we also have

$$
\begin{aligned}
& \lim _{\varepsilon_{1} \rightarrow+0} \lim _{\varepsilon_{2} \rightarrow+0} \cdots \lim _{\varepsilon_{l-1 \rightarrow+0}} \int_{A}^{+\infty e^{-i \kappa \varphi_{l}}} \exp \left(\xi z-\sum_{i=1}^{l-1} \varepsilon_{i} z^{\rho_{i}}\right)\left(\sum_{i=1}^{l-1} u_{i}\left(z^{-1 / \kappa}\right)\right) z^{-1} d z \\
& =\lim _{\varepsilon_{1} \rightarrow+0} \lim _{\varepsilon_{2} \rightarrow+0} \cdots \lim _{\varepsilon_{l-1 \rightarrow+0}} \int_{A}^{+\infty e^{-i \kappa \varphi} l-1} \exp \left(\xi z-\sum_{i=1}^{l-1} \varepsilon_{i} z^{\rho}\right)\left(\sum_{i=1}^{l-1} u_{i}\left(z^{-1 / \kappa}\right)\right) z^{-1} d z \\
& =\lim _{\varepsilon_{1} \rightarrow+0} \lim _{\varepsilon_{2} \rightarrow+0} \cdots \lim _{\varepsilon_{l-2 \rightarrow+0}} \int_{A}^{+\infty e^{-\imath \kappa \varphi} l-1} \exp \left(\xi z-\sum_{i=1}^{l-2} \varepsilon_{i} z^{\rho_{l}}\right)\left(\sum_{i=1}^{l-2} u_{\imath}\left(z^{-1 / \kappa}\right)\right) z^{-1} d z \\
& \quad+\int_{A}^{+\infty e^{-\imath \kappa \varphi_{l-1}}} \exp (\xi z) u_{l-1}\left(z^{-1 / \kappa}\right) z^{-1} d z .
\end{aligned}
$$

Repeating this argument, we have for $\xi$ with $|\arg \xi-\pi|<\theta^{*}$

$$
\hat{u}(\xi)=\sum_{i=1}^{l} \hat{u}_{i}(\xi), \quad \hat{u}_{i}(\xi)=\sum_{i=1}^{l} \int_{A}^{+\infty e^{-\imath \kappa \varphi_{i}}} \exp (\xi z) u_{i}\left(z^{-1 / \kappa}\right) z^{-1} d z
$$

By the assumption $u_{i}(z)$ has the $\kappa$-asymptotic expansion on $\left\{z ; \arg z=\varphi_{i}\right\}$ 。 Hence $\hat{u}_{i}(\xi) \in \mathcal{O}\left(\tilde{\Xi}_{0}\right), \Xi_{0}=\{0<|\xi|<c\}$, for some $c>0$ and (1) and (2) in Proposition 2.4 hold for each $\hat{u}_{i}(\xi)$. Hence $\hat{u}(\xi) \in \mathcal{O}(\boldsymbol{\Xi}(h, a, b))$ has a holomorphic prolongation to $\left\{\xi ;|\arg \xi-\pi|<\theta^{*}\right\} \cup \tilde{\Xi}_{0}$. So the assumptions in Theorem 2.5 hold and the assertions follow.

We give a few propositions for the later sections. The next two mean that holomorphic functions with bounds in a sector are represented as a sum of those in wider sectors.

Proposition 2.11. Let $u(z) \in \mathcal{O}_{(x)}(\Omega(a, b))$. Given $h>0$, there are $u_{1}(z) \in$ $\mathcal{O}_{\{\kappa\}, h}(U(a, b+2 \pi))$ and $u_{2}(z) \in \mathcal{O}_{\{\kappa\}, h}(U(a-2 \pi, b))$ such that $u(z)=u_{1}(z)+u_{2}(z)$ in $U(a, b)$, where $U$ is a neighbourhood of $z=0$. 
Proof. We may assume that $a<0<b$. Given $h>0$, define

$$
U(z)=\frac{\exp \left(h z^{-\kappa} / 3\right)}{2 \pi i} \int_{0}^{r} \frac{\exp \left(-h t^{-\kappa} / 3\right)}{t-z} u(t) d t .
$$

Then, by deforming the integration path, we can prolong $U(z)$ to the $U(a, b+2 \pi)$, $U=\{|z|<r\}$. We denote by $u_{1}(z)$ this extension, $U(z)$ is also considered to be a holomorphic function on $U(a-2 \pi, b)$, say $-u_{2}(z)$. Then we have $u(z)=$ $u_{1}(z)+u_{2}(z)$ on $U(a, b)$ and we can easily show $u_{1}(z) \in \mathcal{O}_{\{\kappa\}, h}(U(a, b+2 \pi))$ and $u_{2}(z) \in \mathcal{O}_{(\kappa), h}(U(a-2 \pi, b))$.

We have by the same method as in Proposition 2.11 .

Proposition 2.12. Let $u(z) \in \mathcal{O}_{(\kappa), h}(\Omega(a, b))$. Given $h^{\prime}>h$, there are $u_{1}(z) \in$ $\mathcal{O}_{\{\kappa\}, h^{\prime}}(U(a, b+2 \pi))$ and $u_{2}(z) \in \mathcal{O}_{\{\kappa\}, h^{\prime}}(U(a-2 \pi, b))$ such that $u(z)=u_{1}(z)+u_{2}(z)$ in $U(a, b), U=\{|r|<r\}$.

We give a relation $A s y_{(r)}(\Omega(a, b))$ between $\tilde{M}-A s y_{(\kappa)}(\Omega(a, b))$.

Proposition 2.13. For $u(z) \in A s y_{(\kappa)}(\Omega(a, b))$ there is a $\tilde{u}(z) \in \tilde{\mathcal{H}}-A s y_{(\kappa)}(U(a, b))$, $U=\{|z|<r\}$, such that $\tilde{u}(z)-u(z) \log z \in A s y_{(\kappa)}(U(a, b))$.

Proof. We may assume that $a=-b=\theta$. Define

$$
\tilde{u}(z)=\int_{0}^{r} \frac{u(t)}{z-t} d t
$$

Then $\tilde{u}(z) \in \mathcal{O}(U(-\theta, \theta+2 \pi)), U=\{|z|<r\}$. Put $w(z)=\int_{0}^{r} \frac{u(t)-u(z)}{t-z} d t$. Then

$$
\tilde{u}(z)=\int_{0}^{r} \frac{u(z)}{z-t} d t+w(z)=u(z)(\log z-\log (z-r))+w(z) .
$$

We show $w(z) \in A s y_{(\kappa)}(U(\theta))$. We have $w(z)=\int_{0}^{r} d t \int_{0}^{1} u^{\prime}(s t+(1-s) z) d s$ and $w^{(n)}(z)=\int_{0}^{r} d t \int_{0}^{1}(1-s)^{n} u^{(n+1)}(s t+(1-s) z) d s$. Hence if $|\operatorname{larg} z|<\theta_{1}<\theta$,

$$
\begin{aligned}
\left|w^{(n)}(z)\right| & \leqq A B^{n+1} \Gamma\left(\frac{n+1}{\kappa}+1\right)(n+1) ! \int_{0}^{r} d t \int_{0}^{1}(1-s)^{n} d s \\
& \leqq A B^{n+1} \Gamma\left(\frac{n+1}{\kappa}+1\right) n !
\end{aligned}
$$

which means $w(z) \in A s y_{(\kappa)}(U(\theta))$. Hence $\tilde{u}(z)-u(z) \log z \in A s y_{(x)}(U(a, b))$. 


\section{§ . Integro-differential Operators Derived from $L\left(z, \partial_{z}\right)$}

In order to show Theorems in $\S 1$ we need integro-differential operators $\mathcal{L}_{\alpha}\left(z, \lambda, \zeta, \partial_{z}, \partial_{\zeta}, \lambda t_{0}-\lambda \partial_{\lambda}\right)(0<\alpha<1)$ containing an integral operator $\partial_{\zeta}^{-1}$, where $z \in C^{n+1}$ and $\lambda, \zeta, t_{0} \in C^{1} . \quad \mathcal{L}_{\alpha}=\mathcal{L}_{\alpha}\left(z, \lambda, \zeta, \partial_{z}, \partial_{\zeta}, \lambda t_{0}-\lambda \partial_{\lambda}\right)$ is derived from $L\left(z, \partial_{z}\right)$ in (1.1) as follows: Let $0<\alpha<1 . L\left(z, \partial_{z}\right)$ is the sum of $L_{k, l}\left(z, \partial_{z}\right)=$ $z_{0}^{j} a_{k, l}\left(z, \partial^{\prime}\right) \partial_{0}^{k-l} \quad\left(j=j(k, l), s_{k} \leqq l \leqq m, 0 \leqq k \leqq m\right) \quad$ (see (1.2)). We correspond $\mathcal{L}_{\alpha, k, l}=\mathcal{L}_{\alpha, k, l}\left(z, \lambda, \zeta, \partial_{z}, \partial_{\zeta}, \lambda t_{0}-\lambda \partial_{\lambda}\right)$ to each $L_{k, l}\left(z, \partial_{z}\right)$,

$$
\mathcal{L}_{a, k, l}=\left\{\lambda^{-1+a}\left(\alpha \zeta+\left(\lambda t_{0}-\lambda \partial_{\lambda}\right) \partial_{\zeta}^{-1}\right)\right\}^{\jmath}\left\{\lambda^{a l} a_{k, l}\left(z, \partial^{\prime} \partial_{\zeta}^{-1}\right)\right\}\left\{\left(\lambda^{\alpha} \partial_{0} \partial_{\zeta}^{-1}+\lambda\right)^{k-l}\right\} .
$$

Namely we get $\mathcal{L}_{\alpha, k, l}$ from $L_{k, l}\left(z, \partial_{z}\right)$ by the following replacements :

$$
z_{0} \longrightarrow \lambda^{-1+\alpha}\left(\alpha \zeta+\left(\lambda t_{0}-\lambda \partial_{\lambda}\right) \partial_{\zeta}^{-1}\right), \quad \partial^{\prime} \longrightarrow \lambda^{\alpha} \partial^{\prime} \partial_{\bar{\zeta}}^{-1}, \quad \partial_{0} \longrightarrow \lambda^{\alpha} \partial_{0} \partial_{\zeta}^{-1}+\lambda,
$$

but the variable $z_{0}$ in $a_{k, l}\left(z, \partial^{\prime}\right)$ is not replaced. Define

$$
\mathcal{L}_{\alpha}=\mathcal{L}_{\alpha}\left(z, \lambda, \zeta, \partial_{z}, \partial_{\zeta}, \lambda t_{0}-\lambda \partial_{\lambda}\right)=\sum_{k=0}^{m} \sum_{l=s_{k}}^{k} \mathcal{L}_{\alpha_{0} k, l} .
$$

Now we define $\partial_{\zeta}^{-1}$ in $\mathcal{L}_{a}$. In order to do so we introduce a sequence of auxiliary functions $\left\{f_{,}(\zeta)\right\}(j \in \boldsymbol{Z})$ used in $[1]$ :

$$
\left\{\begin{array}{l}
f_{J}(\zeta)=\frac{\zeta^{j}}{(2 \pi i) j !}\left\{\log \zeta-\left(1+\frac{1}{2}+\cdots+\frac{1}{j}\right)\right\} \quad(j \geqq 1), \\
f_{0}(\zeta)=\frac{1}{(2 \pi i)} \log \zeta, \\
f_{j}(\zeta)=\frac{(-1)^{j+1}}{2 \pi i}(-j-1) ! \zeta^{j} \quad(j \leqq-1) .
\end{array}\right.
$$

It is easy to show the next lemma.

Lemma 3.1. The following identities hold:

$$
\begin{aligned}
\frac{d}{d \zeta} f_{j}(\zeta) & =f_{j-1}(\zeta), \\
\zeta f_{j}(\zeta) & =(j+1) f_{j+1}(\zeta)+a \text { polynomial of } \zeta .
\end{aligned}
$$

By considering Lemma 3.1, we define

$$
\partial_{\zeta}^{-1} f_{j-1}(\zeta+c)=f_{j}(\zeta+c),
$$

where $c$ does not depend on $\zeta$. By (3.6), we have

$$
\zeta f_{j}(\zeta+c) \equiv(j+1) f_{j+1}(\zeta+c)-c f_{j}(\zeta+c) \quad(\bmod \text {. polynomials of } \zeta) \text {. }
$$

All the calculations with respect to $\zeta$ will be performed by the relations (3.5)-(3.8). Polynomials of $\zeta$ are neglected in all the calculations below. It will turn out that they make no contribution to the integration on the closed 
paths in $\zeta$-space. The notation $\equiv$ means modulo polynomials of $\zeta$ in $\S 3$.

Now we define the operation of $\mathcal{L}_{\alpha}=\mathcal{L}_{a}\left(z, \lambda, \zeta, \partial_{z}, \partial_{\zeta}, \lambda t_{0}-\lambda \partial_{\lambda}\right)$ to a function $V(z, t, \lambda, \zeta, \tau)$ of the form

$$
V(z, t, \lambda, \zeta, \tau)=\sum_{n=n_{0}}^{+\infty} v_{n}(z, t, \lambda, \tau) f_{n}\left(\zeta+\tau z_{1}\right) .
$$

We prepare several lemmas for this operation. $v(z, t, \lambda, \tau)$ in Lemmas $3.2-3.4$ and Proposition 3.5 is holomorphic in some domain.

\section{Lemma 3.2.}

$$
\begin{aligned}
& \left(\lambda^{-1+a} \partial_{0} \partial_{\zeta}^{-1}+1\right)^{s} v(z, t, \lambda, \tau) f_{n}\left(\zeta+\tau z_{1}\right) \\
& \quad=\sum_{r=0}^{s}\left(\begin{array}{l}
s \\
r
\end{array}\right) \lambda^{(-1+a) r} \partial_{0}^{r} v(z, t, \lambda, \tau) f_{n+r}\left(\zeta+\tau z_{1}\right) .
\end{aligned}
$$

Lemma 3.3. There are linear partial differential operators $a_{k, l, s}\left(z, \partial^{\prime}\right)$ $(0 \leqq s \leqq l)$ with ord. $a_{k, l, s}\left(z, \partial^{\prime}\right) \leqq s$ such that

$$
\begin{aligned}
& a_{k, l}\left(z, \partial^{\prime} \partial_{\zeta}^{-1}\right) v(z, t, \lambda, \tau) f_{n}\left(\zeta+\tau z_{1}\right) \\
& \quad=\sum_{s=0}^{l} \tau^{l-s} a_{k, l, s}\left(z, \partial^{\prime}\right) v(z, t, \lambda, \tau) f_{n+s}\left(\zeta+\tau z_{1}\right),
\end{aligned}
$$

where $a_{k, l, 0}(z)=\left.a_{k, l}\left(z, \xi^{\prime}\right)\right|_{\xi^{\prime}=\hat{\xi}^{\prime}=(1,0,0)}$.

Lemma 3.2 follows from the binomial theorem and the proof of Lemma 3.3 is easy.

Lemma 3.4. The following identity holds:

$$
\begin{aligned}
& \left\{\lambda^{-1+a}\left(\alpha \zeta+\left(\lambda t_{0}-\lambda \partial_{\lambda}\right) \partial_{\zeta}^{-1}\right)\right\}^{j} v(z, t, \lambda, \tau) f_{n}\left(\zeta+\tau z_{1}\right) \\
& \equiv \lambda^{(-1+\alpha) j}\left\{\sum_{s=0}^{j}\left(-\alpha \tau z_{1}\right)^{j-s} P_{s}^{j}\left(n, \lambda t_{0}-\lambda \partial_{\lambda}\right) v(z, t, \lambda, \tau) f_{n+s}\left(\zeta+\tau z_{1}\right)\right\} .
\end{aligned}
$$

Here $P_{0}^{j}\left(n, \lambda t_{0}-\lambda \partial_{\lambda}\right)=I$ and $P_{s}^{j}\left(n, \lambda t_{0}-\lambda \partial_{\lambda}\right)=\sum_{k=0}^{s} p_{s, k}^{j}(n)\left(\lambda t_{0}-\lambda \partial_{\lambda}\right)^{k}$, where constants $p_{s, k}^{j}(n)(0 \leqq k \leqq s, 0 \leqq s \leqq j)$ satify for constants $A$ and $B$

$$
\left|p_{s, k}^{j}(n)\right| \leqq A B^{j}(|n|+j+1)^{s-k}, \quad 0 \leqq k \leqq s \leqq \jmath .
$$

Proof. The identity (3.11) is obvious for $j=0$. We have by (3.8)

$$
\begin{aligned}
& \lambda^{-1+a}\left(\alpha \zeta+\left(\lambda t_{0}-\lambda \partial_{\lambda}\right) \partial_{\zeta}^{-1}\right) v(z, t, \lambda, \tau) f_{n}\left(\zeta+\tau z_{1}\right) \\
& \equiv \lambda^{-1+\alpha}\left\{\left(-\alpha \tau z_{1}\right) v(z, t, \lambda, \tau) f_{n}\left(\zeta+\tau z_{1}\right)\right. \\
&\left.+\left(\alpha(n+1)+\left(\lambda t_{0}-\lambda \partial_{\lambda}\right)\right) v(z, t, \lambda, \tau) f_{n+1}\left(\zeta+\tau z_{1}\right)\right\}
\end{aligned}
$$

Thus, by putting $P_{1}^{1}\left(n, \lambda t_{0}-\lambda \partial_{\lambda}\right)=\alpha(n+1)+\left(\lambda t_{0}-\lambda \partial_{\lambda}\right)$, we have (3.11) and (3.12) for $i=1$. Assume (3.11) and (3.12) for some $j$. Then we have 


$$
\begin{aligned}
&\left\{\lambda^{-1+\alpha}\left(\alpha \zeta+\left(\lambda t_{0}-\lambda \partial_{\lambda}\right) \partial_{\zeta}^{-1}\right)\right\}^{j+1} v(z, t, \lambda, \tau) f_{n}\left(\zeta+\tau z_{1}\right) \\
& \equiv \lambda^{-1+\alpha}\left(\alpha \zeta+\left(\lambda t_{0}-\lambda \partial_{\lambda}\right) \partial_{\zeta}^{-1}\right) \\
&\left\{\lambda^{(-1+\alpha) j}\left(\sum_{s=0}^{j}\left(-\alpha \tau z_{1}\right)^{j-s} P_{s}^{j}\left(n, \lambda t_{0}-\lambda \partial_{\lambda}\right) v(z, t, \lambda, \tau) f_{n+s}\left(\zeta+\tau z_{1}\right)\right)\right\} \\
& \equiv \lambda^{-1+\alpha}\left[\left(-\alpha \tau z_{1}\right)\left\{\lambda^{(-1+\alpha) j}\left(\sum_{s=0}^{j}\left(-\alpha \tau z_{1}\right)^{j-s} P_{s}^{j}\left(n, \lambda t_{0}-\lambda \partial_{\lambda}\right) v(z, t, \lambda, \tau) f_{n+s}\left(\zeta+\tau z_{1}\right)\right)\right\}\right. \\
&+\left(\alpha(n+s+1)+\left(\lambda t_{0}-\lambda \partial_{\lambda}\right)\right) \\
&\left.\left\{\lambda^{(-1+\alpha)}\left(\sum_{s=0}^{j}\left(-\alpha \tau z_{1}\right)^{j-s} P_{s}^{j}\left(n, \lambda t_{0}-\lambda \partial_{\lambda}\right) v(z, t, \lambda, \tau) f_{n+s+1}\left(\zeta+\tau z_{1}\right)\right)\right\}\right] \\
& \equiv \lambda^{(-1+\alpha)(j+1)}\left\{\sum_{s=0}^{j}\left(-\alpha \tau z_{1}\right)^{j+1-s} P_{s}^{j}\left(n, \lambda t_{0}-\lambda \partial_{\lambda}\right) v(z, t, \lambda, \tau) f_{n+s}\left(\zeta+\tau z_{1}\right)\right) \\
&+\left(\sum_{s=0}^{j}\left(-\alpha \tau z_{1}\right)^{j-s}(\alpha(n+s+1)+j(1-\alpha)) P_{s}^{j}\left(n, \lambda t_{0}-\lambda \partial_{\lambda}\right) v(z, t, \lambda, \tau) f_{n+s+1}\left(\zeta+\tau z_{1}\right)\right) \\
&\left.+\left(\sum_{s=0}^{j}\left(-\alpha \tau z_{1}\right)^{j-s}\left(\lambda t_{0}-\lambda \partial_{\lambda}\right) P_{s}^{j}\left(n, \lambda t_{0}-\lambda \partial_{\lambda}\right) v(z, t, \lambda, \tau) f_{n+s+1}\left(\zeta+\tau z_{1}\right)\right)\right\} .
\end{aligned}
$$

By putting $P_{s}^{j+1}\left(n, \lambda t_{0}-\lambda \partial_{\lambda}\right)=P_{s}^{j}\left(n, \lambda t_{0}-\lambda \partial_{\lambda}\right)+(\alpha(n+s)+j(1-\alpha)) P_{s-1}^{j}\left(n, \lambda t_{0}-\right.$ $\left.\lambda \partial_{\lambda}\right)+\left(\lambda t_{0}-\lambda \partial_{\lambda}\right) P_{s-1}^{j}\left(n, \lambda t_{0}-\lambda \partial_{\lambda}\right)$, we have (3.11) and (3.12) for $j+1$.

Thus, making full use of the above Lemmas, we have

Proposition 3.5. The following holds:

$$
\begin{aligned}
& \mathcal{L}_{\alpha, k, l} v(z, t, \lambda, \tau) f_{n}\left(\zeta+\tau z_{1}\right) \\
& \equiv \sum_{\substack{0 \leq r \leq k-l \\
0 \leq s \leq l}}\left(\begin{array}{c}
k-l \\
r
\end{array}\right) \lambda^{-(1-\alpha) j}\left\{\sum_{0 \leq d \leq j}\left(-\alpha \tau z_{1}\right)^{j-d} P_{d}^{j}\left(n+s+r, \lambda t_{0}-\lambda \partial_{\lambda}\right)\right. \\
& \left.\lambda^{k-(1-\alpha)(l+r)} \tau^{l-s} a_{k, l, s}\left(z, \partial^{\prime}\right) \partial_{0}^{r} v(z, t, \lambda, \tau)\right\} f_{n+s+r+d}\left(\zeta+\tau z_{1}\right) .
\end{aligned}
$$

Now let us construct a formal solution $V(z, t, \lambda, \zeta, \tau)$ of

$$
\mathcal{L}_{\alpha} V(z, t, \lambda, \zeta, \tau) \equiv F(z, t, \lambda, \tau) f_{n_{0}}\left(\zeta+\tau z_{1}\right),
$$

which has the form (3.9). Define

$$
Q_{l+r, d}^{j, k}\left(n, \lambda t_{0}-\lambda \partial_{\lambda}\right)=\lambda^{-k+(1-\alpha)(l+r)} P_{d}^{j}\left(n, \lambda t_{0}-\lambda \partial_{\lambda}\right) \lambda^{k-(1-\alpha)(l+r)} .
$$

where $0 \leqq k \leqq m, s_{k} \leqq l \leqq k$ and $0 \leqq r \leqq k-l$. By operating $\mathcal{L}_{\alpha}$ to $V(z, t, \lambda, \zeta, \tau)$ and setting the coefficients of the same $f_{N}\left(\zeta+\tau z_{1}\right)$ equal to each other, we have from Proposition 3.5

$$
\begin{aligned}
& \sum_{n+s+r+d=N}\left(\begin{array}{c}
k-l \\
r
\end{array}\right) \lambda^{-(1-\alpha) j}\left\{\sum_{0 \leq d \leq j}\left(-\alpha \tau z_{1}\right)^{j-d} P_{d}^{j}\left(n+s+r, \lambda t_{0}-\lambda \partial_{\lambda}\right)\right. \\
& \left.\lambda^{k-(1-\alpha)(l+r)} \tau^{l-s} a_{k, l, s}\left(z, \partial^{\prime}\right) \partial_{0}^{r} v_{n}(z, t, \lambda, \tau)\right\} \\
& =\sum_{n+s+r+d=N}\left(\begin{array}{c}
k-l \\
r
\end{array}\right) \lambda^{k-(1-\alpha)(l+r+j)}\left\{\sum_{0 \leq d \leq j}\left(-\alpha \tau z_{1}\right)^{j-d}\right. \\
& \left.Q_{l+r, a}^{j, k}\left(n+s+r, \lambda t_{0}-\lambda \partial_{\lambda}\right) \tau^{l-s} a_{k, l, s}\left(z, \partial^{\prime}\right) \partial_{0}^{r} v_{n}(z, t, \lambda, \tau)\right\}=\delta_{N, n_{0}} F(z, t, \lambda) .
\end{aligned}
$$


In order to show Theorems in $\S 1$ we'll put $\alpha=\alpha_{1}=\left(\sigma_{1}-1\right) / \sigma_{1}$ or $\alpha=\alpha_{p-1}=$ $\left(\sigma_{p-1}-1\right) / \sigma_{p-1}$. So we give a lemma for later purposes.

Lemma 3.6. Assume $d_{k, l}=l+j(k, l) \neq+\infty$. Then there are nonnegative $\boldsymbol{\beta}_{k, l}^{j} \in \boldsymbol{Q}(1 \leqq i \leqq p-1)$ such that

$$
\left(d_{k_{i-1}}-d_{k, l}\right)\left(1-\alpha_{i}\right)+\beta_{k, l}^{j}=k_{i-1}-k,
$$

$\left(k, d_{k, l}\right) \in \Sigma(i)$ if and only if $\beta_{k, l}^{i}=0$, and

$$
\left\{\begin{array}{l}
\left(d_{k_{i}}-d_{k, l}\right)\left(\alpha_{1}-\alpha_{i}\right)+\beta_{k_{i}}^{1}-\beta_{k_{,} l}^{1}+\beta_{k_{,}}^{i}=0, \\
d_{k_{i-1}}\left(\alpha_{1}-\alpha_{i}\right)+\beta_{k_{i-1}}^{1}=d_{k_{i}}\left(\alpha_{1}-\alpha_{i}\right)+\beta_{k_{i}}^{1},
\end{array}\right.
$$

where $\beta_{k_{i}}^{1}=\beta_{k_{i}, l_{k_{i}}}^{1}($ see (1.3)).

Lemma 3.6 follows from the lower convexity of $\Sigma$ and the definition of $\sum(i)$ and $d_{k_{i}}$ (see [12]). Now put $\alpha=\alpha_{\imath}=\left(\sigma_{i}-1\right) / \sigma_{i}(1 \leqq i \leqq p-1)$. From Lemma 3.6, $k-d_{k, l}\left(1-\alpha_{i}\right)=k_{i-1}-d_{k_{i-1}}\left(1-\alpha_{i}\right)-\beta_{k_{1} l}^{i}$. Hence we have from (3.16),

$$
\begin{aligned}
& \lambda^{k i-1-\left(1-\alpha_{\imath}\right) d_{k i-1}} \sum_{n+s+r+d=N}\left(\begin{array}{c}
k-l \\
r
\end{array}\right) \lambda^{-\left(1-\alpha_{i}\right) r-\beta_{k, l}^{i}} \\
& \left\{\sum_{0 \leqq d \leqq j}\left(-\alpha_{i} z_{1}\right)^{j-d} Q_{k+r, d}^{j, k}\left(n+s+r, \lambda t_{0}-\lambda \partial_{\lambda}\right) \tau^{d_{k, l}-s-d} a_{k, l, s}\left(z, \partial^{\prime}\right) \partial_{0}^{r} v_{n}(z, t, \lambda, \tau)\right\} \\
& =\delta_{N, n_{0}} F(z, t, \lambda) \text {. }
\end{aligned}
$$

Put

$$
\begin{aligned}
& G_{q}^{2}\left(z, \partial^{\prime}, \lambda, \tau\right)=\lambda^{k_{\imath-1}-\left(1-a_{\imath}\right) d_{k i-1}}
\end{aligned}
$$

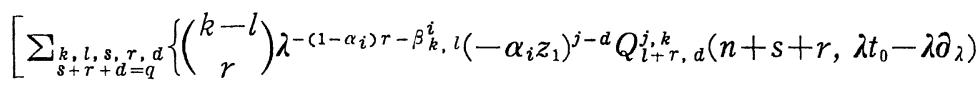

$$
\begin{aligned}
& \left.\left.\tau^{d k, 1-s-d} a_{k, l, s}\left(z, \partial^{\prime}\right) \partial_{0}^{r}\right\}\right] \text {. }
\end{aligned}
$$

$G_{0}^{i}\left(z, \partial^{\prime}, \lambda, \tau\right)$ is a polynomial of $\tau$. So we denote it by $G_{0}^{i}(z, \lambda, \tau)$, which will often appear and has the form

$$
G_{0}^{i}(z, \lambda, \tau)=\lambda^{k_{i-1}-\left(1-\alpha_{i}\right) d_{k i-1}}\left\{\sum_{k, l} \lambda^{-\beta_{k, l}^{i}}\left(-\alpha_{1} z_{1}\right)^{j} \tau_{k, l}^{d} a_{k, l}(z)\right\},
$$

where $a_{k, l}(z)=a_{k, l, 0}(z)=a_{k, l}\left(z, \hat{\xi}^{\prime}\right)$ (see Lemma 3.3). Thus the equation (3.19) becomes

$$
\begin{aligned}
& G_{0}^{i}(z, \lambda, \tau) v_{N}(z, t, \lambda, \tau) \\
& \quad+\Sigma_{q} G_{q}^{i}\left(z, \partial^{\prime}, \lambda, \tau\right) v_{N-q}(z, t, \lambda, \tau)=\delta_{N, n_{0}} F(z, t, \lambda, \tau),
\end{aligned}
$$

where $\Sigma_{q}$ is a finite sum. Consequently we can determine $v_{N}(z, t, \lambda, \tau)\left(N \geqq n_{0}\right)$ successively by (3.22). In order to determine them we need the division by 
$G_{0}^{i}(z, \lambda, \tau)$. So the properties and the estimates of $v_{N}(z, t, \lambda, \tau)$ depend on the zeros of $G_{0}^{i}(z, \lambda, \tau)$. In particular, if $F(z, t, \lambda, \tau)$ is rational in $\tau, v_{N}(z, t, \lambda, \tau)$ $\left(N \geqq n_{0}\right)$ are also rational functions of $\tau$. $G_{0}^{p-1}(z, \lambda, \tau)$ is simple. The zeros of $G_{0}^{1}(z, \lambda, \tau)$ are studied in $\S 6$.

\section{§4. Construction of Kernels $G(w, z, t)$ and $G_{R}(w, z, t)$}

In $\S 4$ we construct $G(w, z, t)$ and $G_{R}(w, z, t)$ in Theorem 1.11 by using the results in $\S 3$. So we assume (1.17)-(a), (b), (c) in this section. Now put $\alpha=$ $\alpha_{p-1}$ and $\tau=0$ in (3.14). Consider

$$
\mathcal{L}_{\alpha} V(z, t, \lambda, \zeta)=F(z, t, \lambda) f_{-1}(\zeta),
$$

where

$$
F(z, t, \lambda)=\frac{-1}{(2 \pi i)^{n}} \Pi_{i=1}^{n} \frac{1}{\left(t_{i}-z_{i}\right)}
$$

Since $\alpha=\alpha_{p-1}, \tau=0$ and $d_{k-1}=0$, we have from (3.21)

$$
\begin{aligned}
& G_{0}(z, \lambda)=G_{0}^{p-1}(z, \lambda, 0)=\lambda^{k} p-1 \sum_{(k ; j(k, 0)=0 ;} \lambda^{-\beta_{k, 0}^{p-1}} a_{k, 0}(z) \\
& =\lambda^{k} p-1\left(a_{k p-1,0}(z)+\sum_{(k ; k \neq k}{ }_{p-1}, \jmath(k, 0)=0\right) \\
& \left.\lambda^{-\beta_{k, 0}^{p-1}} a_{k, 0}(z)\right) .
\end{aligned}
$$

If $k \neq k_{p-1}, \beta_{k, 0}^{p-1}>0$ in (4.3). By the assumption (1.17)-(c) we have $\left|G_{0}(z, \lambda)\right|$ $\geqq C|\lambda|^{k p-1}, \quad C>0$, for large $\lambda$ and in a neighbourhood of $z=0$. So we can construct a formal solution $V(z, t, \lambda, \zeta)$ of (4.1) by the method in $\S 3$,

$$
V(z, t, \lambda, \zeta)=\sum_{n=-1}^{+\infty} v_{n}(z, t, \lambda) f_{n}(\zeta) .
$$

$v_{n}(z, t, \lambda)(n \geqq-1)$ are successively determined by the formula (3.22) as holomorphic functions in a neighbourhood of $z=0$. We have

Proposition 4.1. For small $r_{1}, r_{2}, r_{3}\left(0<r_{1}<r_{2}<r_{3}\right)$ and large $\Lambda_{0}$, there exist constants $A$ and $B$ such that

$$
\left|\lambda^{k} p-1 v_{n}(z, t, \lambda)\right| \leqq A B^{n+1}\left(\sum_{r=0}^{n+1}\left|\lambda t_{0}\right|^{r} / r !\right)(n+1) !
$$

holds in $\left\{(z, t, \lambda) ;|z| \leqq r_{1},\left|t_{0}\right| \leqq r_{3}, r_{2} \leqq\left|t_{i}\right| \leqq r_{3}(1 \leqq i \leqq n),|\lambda| \geqq \Lambda_{0}\right\}$.

The proof of Proposition 4.1 is given in $\S 7$. As for the convergence of $V(z, t, \lambda, \zeta)$, we have

Proposition 4.2. $V(z, t, \lambda, \zeta)=\sum_{n=-1}^{+\infty} v_{n}(z, t, \lambda) f_{n}(\zeta)$ converges in $\{(z, t, \lambda, \zeta)$; $\left.|z| \leqq r_{1},\left|t_{0}\right| \leqq r_{3}, r_{2} \leqq\left|t_{i}\right| \leqq r_{3}(1 \leqq i \leqq n),|\lambda| \geqq \Lambda_{0}, 0<|\zeta| \leqq r_{4}\right\}$ for some $r_{4}>0$, the singularity at $\zeta=0$ of $V(z, t, \lambda, \zeta)-v_{-1}(z, t, \lambda) f_{-1}(\zeta)$ is logarithmic and

$$
\left|V(z, t, \lambda, \zeta)-v_{-1}(z, t, \lambda) f_{-1}(\zeta)\right| \leqq A|\lambda|^{q^{*}} \exp \left(C^{*}\left|\lambda \zeta t_{0}\right|\right)(|\log \zeta|+B)
$$


for some constants $A, B, C^{*}$ and $q^{*}$.

Proof. By Proposition 4.1, $\left|\lambda^{k} p-1\left(V(z, t, \lambda, \zeta)-v_{-1}(z, t, \lambda) f_{-1}(\zeta)\right)\right| \leqq A(|\log \zeta|$ $+1) \sum_{n=0}^{+\infty}(B|\zeta|)^{n}\left(\sum_{r=0}^{n+1}\left|\lambda t_{0}\right|^{r} / r\right.$ !). If $2|\zeta| \leqq B^{-1}, \sum_{n=0}^{+\infty}(B|\zeta|)^{n}\left(\sum_{r=0}^{n+1}\left|\lambda t_{0}\right|^{r} / r !\right) \leqq$ $A+\sum_{r=1}^{+\infty}\left(\left|\lambda t_{0}\right|^{r} / r !\left(\sum_{n=r-1}^{+\infty}(B|\zeta|)^{n}\right)\right) \leqq A\left(1+\sum_{\tau=1}^{+\infty}\left|\lambda t_{0}\right|^{r}|B \zeta|^{r-1} / r !\right) \leqq A|\lambda| \exp \left(B\left|\lambda \zeta t_{0}\right|\right)$. So we have (4.6).

The constants $C^{*}$ and $q^{*}$ appering in this section are those in Proposition 4.2. Define paths in $\zeta$-space : $Z\left(\phi, \phi^{\prime}\right)=\left\{\zeta=d^{*} e^{i\left((1-s) a \varphi^{\prime}+s a \psi^{\prime}\right)} ; 0 \leqq s \leqq 1\right\}$ and $Z(\phi)$ $=Z(\psi, \phi+2 \pi / \alpha)$, where $d^{*}>0$. Put

$$
\hat{V}(\psi ; z, t, \lambda)=\int_{z(\psi)} \exp \left(-\lambda^{\alpha} \zeta\right) V(z, t, \lambda, \zeta) d \zeta,
$$

where $0<d^{*} \leqq r_{4}, r_{4}$ being that in Proposition 4.2 , and

$$
\hat{V}\left(\phi, \psi^{\prime} ; z, t, \lambda\right)=\hat{V}(\phi ; z, t, \lambda)-\hat{V}\left(\psi^{\prime} ; z, t, \lambda\right),
$$

where $\left|\alpha\left(\psi-\psi^{\prime}\right)\right|<\pi$. In the definition of $\hat{V}(\psi ; z, t, \lambda)$, the holomorphic part of $V(z, t, \lambda, \zeta)$ as a function of $\zeta$ can be neglected. We have

Lemma 4.3. The following estimates hold:

$$
|\hat{V}(\psi ; z, t, \lambda)| \leqq A|\lambda|^{q^{\sim}} \exp \left(d^{*}|\lambda|^{\alpha}+C^{*} d^{*}\left|\lambda t_{0}\right|\right) .
$$

If $|\arg \lambda+\phi|<\pi / 2 \alpha$,

$$
|\hat{V}(\psi ; z, t, \lambda)| \leqq A|\lambda|^{q^{*}} \exp \left(C^{*} d^{*}\left|\lambda t_{0}\right|\right) .
$$

If $-(\pi / 2 \alpha)+\left(\left|\psi-\psi^{\prime}\right| / 2\right)+\varepsilon<\arg \lambda+\left(\phi+\psi^{\prime}\right) / 2<(\pi / 2 \alpha)-\left(\left|\phi-\psi^{\prime}\right| / 2\right)-\varepsilon$, where $0<$ $\alpha \varepsilon<(\pi / 2)-\left(\alpha\left|\psi-\psi^{\prime}\right| / 2\right)$,

$$
\left|\hat{V}\left(\psi, \phi^{\prime} ; z, t, \lambda\right)\right| \leqq A|\lambda|^{q^{*}} \exp \left(C^{*} d^{*}\left|\lambda t_{0}\right|-d^{*} \sin (\alpha \varepsilon)|\lambda|^{\alpha}\right) .
$$

Proof. $\hat{V}(\psi ; z, t, \lambda)$ is well defined and we have (4.9) and (4.10) from (4.6) and the deformation of the integration path $Z(\psi)$. We have

$$
\begin{aligned}
& \hat{V}\left(\phi, \psi^{\prime} ; z, t, \lambda\right)=\left(\int_{Z\left(\psi^{\prime}\right)}-\int_{Z\left(\psi^{\prime}\right)}\right) \exp \left(-\lambda^{a} \zeta\right) V(z, t, \lambda, \zeta) d \zeta \\
& =\left(\int_{Z\left(\psi^{\prime}+2 \pi / \alpha, \psi+2 \pi / a\right)}+\int_{Z\left(\psi^{\prime}, \psi^{\prime}\right)}\right) \exp \left(-\lambda^{\alpha} \zeta\right) V(z, t, \lambda, \zeta) d \zeta
\end{aligned}
$$

and $\operatorname{Re} \lambda^{a} \zeta \geqq d^{*} \sin (\alpha \varepsilon)|\lambda|^{a}$ on $Z\left(\psi+2 \pi / \alpha, \psi^{\prime}+2 \pi / \alpha\right) \cup Z\left(\psi, \psi^{\prime}\right)$. Thus we have (4.11).

By Lemma 4.3 we can define

$$
G(\psi ; w, z, t)=\int_{\Lambda_{0}}^{\infty \imath \varphi} \exp (-\lambda w) \hat{V}(\psi ; z, t, \lambda) d \lambda
$$


and

$$
\begin{aligned}
& G\left(\psi, \psi^{\prime} ; w, z, t\right)=G(\psi ; w, z, t)-G\left(\psi^{\prime} ; w, z, t\right) \\
& =\int_{\Lambda_{0}}^{\infty} e^{i \varphi} \exp (-\lambda w) \hat{V}\left(\psi, \psi^{\prime} ; z, t, \lambda\right) d \lambda .
\end{aligned}
$$

Put, by using the constants in Proposition 4.1,

$$
\left\{\begin{array}{l}
X=\left\{(z, t) ;|z| \leqq r_{1},\left|t_{0}\right| \leqq r_{3}, r_{2} \leqq|t| \leqq r_{3} \quad(1 \leqq i \leqq n)\right\}, \\
\Omega=\left\{z ;|z| \leqq r_{3}\right\}, \quad U=\left\{z ;|z| \leqq r_{1}\right\}, \\
X_{\Lambda_{0}}=X \times \tilde{\Lambda}_{0}^{*}, \quad \Lambda_{0}^{*}=\left\{\lambda ;|\lambda| \geqq \Lambda_{0}\right\},
\end{array}\right.
$$

and recall (see $\S 1$ )

$$
W_{\delta}=\left\{(w, z, t) ;|w|>(\sin \delta)\left|t_{0}\right|,(z, t) \in X\right\} \quad \text { for a small } \delta>0,
$$

the function space $K\left(W_{\hat{o}}\right)$ (see Definition 1.10), and

$$
\alpha=\alpha_{p-1}=\left(\sigma_{p-1}-1\right) / \sigma_{p-1}, \quad \gamma=\sigma_{p-1}-1=\alpha /(1-\alpha) .
$$

We choose $d^{*}>0$ small in the following in this paper, if necessary. We have

Proposition 4.4. (1) $G(\psi ; w, z, t) \in K\left(W_{\delta^{*}}\right), \delta^{*}=\sin ^{-1} C^{*} d^{*}$.

(2) Suppose $|\arg w-\phi|<\pi / 2 \alpha+\pi / 2-\varepsilon$ for small $\varepsilon>0$. Then if $C^{*} d^{*}\left|t_{0}\right|$ $<(\sin \varepsilon)|w|$,

$$
\left|\partial_{w}^{q} \partial_{z}^{r} G(\psi ; w, z, t)\right| \leqq \frac{A B^{q} C^{r} q ! r !}{\left((\sin \varepsilon)|w|-C^{*} d^{*}\left|t_{0}\right|\right)^{q+q^{*+1}}}
$$

(3) Suppose $|\arg w-\phi|<\pi$. Then there is $0<c^{*} \leqq 1$ such that if $C^{*} d^{*}\left|t_{0}\right|$ $<c^{*}|w|$,

$$
\left|\partial_{w}^{q} \partial_{z}^{r} G(\psi ; w, z, t)\right| \leqq \frac{A B^{q} C^{r} q ! r !}{\left(c^{*}|w|-C^{*} d^{*}\left|t_{0}\right|\right)^{q+q^{*+1}}} .
$$

Constants $A, B$ and $C$ in (4.18)-(4.19) are some constants and $q^{*}$ is that in (4.6) (or (4.10)), and $\partial_{z}^{r}$ stands for the $r$-th derivative with respect to $z$.

Proof. By varying $\varphi$ in (4.13), we have the holomorphic prolongation of $G(\psi ; w, z, t)$ with respect to $w$ and $G(\psi ; w, z, t) \in K\left(W_{i^{*}}\right)$ by (4.9). Suppose $|\arg w-\phi|<\pi / 2 \alpha+\pi / 2-\varepsilon$. Then we can choose $\lambda=|\lambda| e^{i \varphi} \quad(|\varphi+\phi|<\pi / 2 \alpha)$ such that $\operatorname{Re} \lambda w \geqq(\sin \varepsilon)|\lambda w|$. Hence, if $C^{*} d^{*}\left|t_{0}\right|<(\sin \varepsilon)|w|$,

$$
\begin{aligned}
& \left|\partial_{w}^{q} G(\psi ; w, z, t)\right| \leqq A \int_{\Lambda_{0}}^{\infty e^{i \varphi}} \exp \left(-(\sin \varepsilon)|\lambda w|+C^{*} d^{*}\left|\lambda t_{0}\right|\right)|\lambda|^{q+q^{*}}|d \lambda| \\
& \quad \leqq A B^{q} q !\left((\sin \varepsilon)|w|-C^{*} d^{*}\left|t_{0}\right|\right)^{-q-q^{*-1}} .
\end{aligned}
$$


We have also the similar estimates for $\partial_{w}^{q} \partial_{z}^{r} G(\phi ; w, z, t)$. In particular suppose $|\arg w-\psi|<\pi$. Then we can choose $\lambda=|\lambda| e^{i \varphi}(|\varphi+\psi|<\pi / 2 \alpha)$ so that $\operatorname{Re} \lambda w$ $\geqq c^{*}|\lambda w|$, where $0<c^{*} \leqq 1$ is determined by $\alpha$. Hence, if $C^{*} d^{*}\left|t_{0}\right|<c^{*}|w|$,

$$
\begin{aligned}
& \left|\partial_{w}^{q} G(\psi ; w, z, t)\right| \leqq A \int_{\Lambda_{0}}^{\infty e^{i \varphi}} \exp \left(-c^{*}|\lambda w|+C^{*} d^{*}\left|\lambda t_{0}\right|\right)|\lambda|^{q+q^{*}}|d \lambda| \\
& \quad \leqq A B^{q} q !\left(c^{*}|w|-C^{*} d^{*}\left|t_{0}\right|\right)^{-q-q^{*-1}} .
\end{aligned}
$$

Thus we have (4.19).

We study $G(\phi ; w, z, t)$ in $\S 4$ and $\S 5$. We calculate $L\left(z, \partial_{z}\right) G\left(\psi ; t_{0}-z_{0}, z, t\right)$ in this section. In the following $\equiv$ means modulo holomorphic functions on $X$ (see (4.15)). We have

\section{Proposition 4.5.}

$$
\begin{aligned}
& L\left(z, \partial_{z}\right) G\left(\psi ; t_{0}-z_{0}, z, t\right) \\
& \equiv \frac{-1}{(2 \pi i)^{n+1}} \Pi_{i=0}^{n}\left(t_{i}-z_{i}\right)^{-1}+G_{R}\left(\psi ; t_{0}-z_{0}, z, t\right),
\end{aligned}
$$

where

$$
\left.G_{R}(\psi ; w, z, t)=\int_{\Lambda_{0}}^{\infty i \varphi} \exp \left(-\lambda w-\lambda^{a} d^{*} e^{i a \dot{\psi}}\right)\right) V_{R}(\psi ; z, t, \lambda) d \lambda
$$

and $V_{R}(\psi ; z, t, \lambda) \in \mathcal{O}\left(X_{\Lambda_{0}}\right)$ with

$$
\left|V_{R}(\phi ; z, t, \lambda)\right| \leqq A(1+|\lambda|)^{N} \exp \left(C^{*} d^{*}\left|\lambda t_{0}\right|\right) \quad \text { for some } N .
$$

Before the proof of Proposition 4.5 we note the following identity:

$$
\begin{aligned}
& L_{k, l}(z, \partial) G\left(\psi ; t_{0}-z_{0}, z, t\right)=z_{0}{ }^{j} a_{k, l}\left(z, \partial^{\prime}\right) \partial_{0}^{k-l} G\left(\psi ; t_{0}-z_{0}, z, t\right) \\
& =\int_{\Lambda_{0}}^{\infty e^{i \varphi}} \exp \left(-\lambda\left(t_{0}-z_{0}\right) z_{0}^{j} d \lambda \int_{Z(\psi)} a_{k, l}\left(z, \partial^{\prime}\right)\left(\partial_{0}+\lambda\right)^{k-l} \exp \left(-\lambda^{\alpha} \zeta\right) V(z, t, \lambda, \zeta) d \zeta\right. \\
& =\int_{\Lambda_{0}}^{\infty e^{i \varphi}}\left(\left(\partial_{\lambda}\right)^{j} \exp \left(\lambda z_{0}\right)\right) \exp \left(-\lambda t_{0}\right) d \lambda \\
& \quad \times \int_{Z(\dot{\psi})} a_{k, l}\left(z, \partial^{\prime}\right)\left(\partial_{0}+\lambda\right)^{k-l} \exp \left(-\lambda^{a} \zeta\right) V(z, t, \lambda, \zeta) d \zeta .
\end{aligned}
$$

Now we give several lemmas to show Proposition 4.5, in which the same notation $\tilde{V}(z, t, \lambda)$ means several functions on $X_{A_{0}}$.

\section{Lemma 4.6.}




$$
\begin{aligned}
& \int_{Z(\psi)}\left(\partial_{0}+\lambda\right)^{k-l} \exp \left(-\lambda^{\alpha} \zeta\right) V(z, t, \lambda, \zeta) d \zeta \\
& =\lambda^{k-l} \int_{Z(\psi)} \exp \left(-\lambda^{\alpha} \zeta\right)\left(\lambda^{-1+\alpha} \partial_{0} \partial_{\zeta^{-1}}+1\right)^{k-l} V(z, t, \lambda, \zeta) d \zeta \\
& \quad+\exp \left(-\lambda^{\alpha} d^{*} e^{i \alpha \dot{\psi}}\right) \tilde{V}(z, t, \lambda),
\end{aligned}
$$

where $|\tilde{V}(z, t, \lambda)| \leqq A(1+|\lambda|)^{N} \exp \left(C^{*} d^{*}\left|\lambda t_{0}\right|\right)$ for some $N$.

Proof. Since $\partial_{\zeta} \exp \left(-\lambda^{a} \zeta\right)=-\lambda^{a} \exp \left(-\lambda^{\alpha} \zeta\right)$ and $\left(\partial_{0}+\lambda\right)^{k-l}=\lambda^{k-l}\left(\lambda^{-1+\alpha} \lambda^{-a} \partial_{0}\right.$ $+1)^{k-l}$, we have (4.23) by integration by parts with respect to $\zeta$.

\section{Lemma 4.7.}

$$
\begin{aligned}
& \int_{Z(\psi)} a_{k, l}\left(z, \partial^{\prime}\right)\left(\partial_{0}+\lambda\right)^{k-l}\left(\exp \left(-\lambda^{\alpha} \zeta\right) V(z, t, \lambda, \zeta)\right) d \zeta \\
& =\lambda^{k-l+a l} \int_{Z\left(\psi^{\prime}\right)} \exp \left(-\lambda^{a} \zeta\right) a_{k, l}\left(z, \partial^{\prime} \partial_{\zeta}^{-1}\right)\left(\lambda^{-1+a} \partial_{0} \partial_{\zeta}^{-1}+\lambda\right)^{k-l} V(z, t, \lambda, \zeta) d \zeta \\
& \quad+\exp \left(-\lambda^{a} d^{*} e^{i \alpha \dot{\psi}}\right) \tilde{V}(z, t, \lambda),
\end{aligned}
$$

where $|\tilde{V}(z, t, \lambda)| \leqq A(1+|\lambda|)^{N} \exp \left(C^{*} d^{*}\left|\lambda t_{0}\right|\right)$ for some $N$.

Proof. We have (4.24) with another $\tilde{V}(z, t, \lambda)$ and another $N$ in the same way as in Lemma 4.6 , by using integration by parts in $\zeta$.

Put

$$
V_{k, l}^{*}(z, t, \lambda, \zeta)=\lambda^{k-l+a l} a_{k, l}\left(z, \partial^{\prime} \partial_{\zeta}^{-1}\right)\left(\lambda^{-1+a} \partial_{0} \partial_{\zeta}{ }^{-1}+\lambda\right)^{k-l} V(z, t, \lambda, \zeta) .
$$

Lemma 4.8. The following identity holds:

$$
\begin{aligned}
\int_{\Lambda_{0}}^{\infty e^{i \varphi}}\left(\left(\partial_{\lambda}\right)^{j} \exp \left(\lambda z_{0}\right)\right) \exp \left(-\lambda t_{0}\right) d \lambda \int_{Z(\psi)} \exp \left(-\lambda^{a} \zeta\right) V_{k, l}^{*}(z, t, \lambda, \zeta) d \zeta \\
\equiv \int_{\Lambda_{0}}^{\infty e^{i \varphi}} \exp \left(-\lambda\left(t_{0}-z_{0}\right)\right) d \lambda \\
\quad \times \int_{z(\psi)} \exp \left(-\lambda^{\alpha} \zeta\right)\left(\lambda^{-1+a}\left(\alpha \zeta+\left(\lambda t_{0}-\lambda \partial_{\lambda}\right) \partial_{\zeta}^{-1}\right)^{j} V_{k, l}^{*}(z, t, \lambda, \zeta) d \zeta\right. \\
\quad+\int_{\Lambda_{0}}^{\infty e^{i \varphi}} \exp \left(-\lambda\left(t_{0}-z_{0}\right)-\lambda^{\alpha} d^{*} e^{i \alpha \psi}\right) \tilde{V}(z, t, \lambda) d \lambda,
\end{aligned}
$$

where $|\tilde{V}(z, t, \lambda)| \leqq A(1+|\lambda|)^{N} \exp \left(C^{*} d^{*}\left|\lambda t_{0}\right|\right)$ for some $N$.

Proof. By integration by parts in $\lambda$, we have 


$$
\begin{aligned}
& \int_{I_{0}}^{\infty e^{i \varphi}}\left(\left(\partial_{\lambda}\right)^{\jmath} \exp \left(\lambda z_{0}\right)\right) \exp \left(-\lambda t_{0}\right) d \lambda \int_{Z(\zeta)} \exp \left(-\lambda^{a} \zeta\right) V_{k, l}^{*}(z, t, \lambda, \zeta) d \zeta \\
& \equiv \int_{\Lambda_{0}}^{\infty e^{2 \zeta}} \exp \left(\lambda z_{0}\right) d \lambda\left(-\partial_{\lambda}\right)^{j}\left\{\exp \left(-\lambda t_{0}\right) \int_{Z\left(\zeta^{\prime}\right)} \exp \left(-\lambda^{\alpha} \zeta\right) V_{k_{2}}^{*} l(z, t, \lambda, \zeta) d \zeta\right\} \\
& \equiv \int_{\Lambda_{0}}^{\infty e^{l \varphi}} \exp \left(-\lambda\left(t_{0}-z_{0}\right)\right) d \lambda \int_{Z\left(\zeta^{\prime}\right)} \exp \left(-\lambda^{\alpha} \zeta\right)\left(\alpha \lambda^{a-1} \zeta+t_{0}-\partial_{\lambda}\right)^{\jmath} V_{k, l}^{*}(z, t, \lambda, \zeta) d \zeta .
\end{aligned}
$$

By integrations by parts in $\zeta$ we have

$$
\begin{aligned}
& \int_{Z(\dot{\varphi})} \exp \left(-\lambda^{a} \zeta\right)\left(\alpha \lambda^{\alpha-1} \zeta+t_{0}-\partial_{\lambda}\right)^{\jmath} V_{k, l}^{*}(z, t, \lambda, \zeta) d \zeta \\
& =\int_{Z(\psi)} \exp \left(-\lambda^{a} \zeta\right)\left(\lambda^{-1+a}\left(\alpha \zeta+\left(\lambda t_{0}-\lambda \partial_{\lambda}\right) \partial_{\zeta}^{-1}\right)^{\jmath} V_{k, l}^{*}(z, t, \lambda, \zeta) d \zeta\right. \\
& \quad+\exp \left(-\lambda^{\alpha} d^{*} e^{i a \dot{\varphi}}\right) \tilde{V}(z, t, \lambda) .
\end{aligned}
$$

Hence we have (4.26).

Summing up Lemmas 4.6-4.8, we have from (3.1)

Lemma 4.9. The following holds:

$$
\begin{aligned}
& L_{k, l}(z, \partial) G\left(\psi ; t_{0}-z_{0}, z, t\right) \equiv \int_{\lambda_{0}}^{\infty e^{2 \varphi}} \exp \left(-\lambda\left(t_{0}-z_{0}\right)\right) d \lambda \int_{Z\left(\zeta^{\prime}\right)} \exp \left(-\lambda^{a} \zeta\right) \\
& \mathcal{L}_{n, k, l} V(z, t, \lambda, \zeta) d \zeta+\int_{I_{0}}^{\infty i \zeta} \exp \left(-\lambda\left(t_{0}-z_{0}\right)-\lambda^{a} d^{*} e^{i n \dot{\varphi}}\right) \tilde{V}(z, t, \lambda) d \lambda,
\end{aligned}
$$

where $|\tilde{V}(z, t, \lambda)| \leqq A(1+|\lambda|)^{N} \exp \left(C^{*} d^{*}\left|\lambda t_{0}\right|\right)$ for some $N$.

Now we can give the proof of Proposition 4.5.

Proof of Proposition 4.5. We have, by Lemma 4.9,

$$
\begin{aligned}
& L\left(z, \partial_{z}\right) G\left(\psi ; z_{0}-t_{0}, z, t\right) \equiv \int_{\Lambda_{0}}^{\infty e^{i \varphi}} \exp \left(-\lambda\left(t_{0}-z_{0}\right)\right) d \lambda \\
& \int_{z(\psi)} \exp \left(-\lambda^{a} \zeta\right) \mathcal{L}_{a}\left(z, \lambda, \zeta, \partial_{z}, \partial_{\zeta}, \lambda t_{0}-\lambda \partial_{\lambda}\right) V(z, t, \lambda, \zeta) d \zeta \\
& +\int_{\Lambda_{0}}^{\infty e^{i \varphi}} \exp \left(-\lambda\left(t_{0}-z_{0}\right)-\lambda^{a} d^{*} e^{i \alpha \psi}\right) V_{R}(\psi ; z, t, \lambda) d \lambda,
\end{aligned}
$$

where $\left|V_{R}(\psi ; z, t, \lambda)\right| \leqq A(1+|\lambda|)^{N} \exp \left(C^{*} d^{*}\left|\lambda t_{0}\right|\right)$ for some $N$. Define

$$
\left.G_{R}(\phi ; w, z, t)=\int_{\Lambda_{0}}^{\infty i \varphi} \exp \left(-\lambda w-\lambda^{a} d^{*} e^{i a \varphi}\right)\right) V_{R}(\phi ; z, t, \lambda) d \lambda .
$$

Then, from (4.1), we have 


$$
\begin{aligned}
& L\left(z, \partial_{z}\right) G\left(\psi ; t_{0}-z_{0}, z, t\right) \\
& \equiv \int_{\Lambda_{0}}^{\infty e^{i \varphi}} \exp \left(-\lambda\left(t_{0}-z_{0}\right)\right) d \lambda \frac{-1}{(2 \pi i)^{n}} \Pi_{i=1}^{n}\left(t_{i}-z_{i}\right)^{-1} \int_{z(\psi)} \exp \left(-\lambda^{\alpha} \zeta\right) f_{-1}(\zeta) d \zeta \\
& \quad+G_{R}\left(\psi ; t_{0}-z_{0}, z, t\right) \\
& \equiv \int_{\Lambda_{0}}^{\infty e^{i \varphi}} \exp \left(-\lambda\left(t_{0}-z_{0}\right)\right) d \lambda \frac{-1}{(2 \pi i)^{n}} \Pi_{i=1}^{n}\left(t_{i}-z_{i}\right)^{-1}+G_{R}\left(\psi ; t_{0}-z_{0}, z, t\right) \\
& \equiv \frac{-1}{(2 \pi i)^{n+1}} \Pi_{i=0}^{n}\left(t_{i}-z_{i}\right)^{-1}+G_{R}\left(\psi ; z_{0}-t_{0}, z, t\right) .
\end{aligned}
$$

We have shown in Proposition 4.4 that $G(\phi ; w, z, t) \in \kappa\left(W_{\delta *}\right), \delta^{*}=\sin ^{-1} C^{*} d^{*}$. It is obvious that $G_{R}(\psi ; w, z, t)$ and $G\left(\phi, \psi^{\prime} ; w, z, t\right)$ are also in $\kappa\left(W_{\delta^{*}}\right)$. In the next section we show that $G(\psi ; w, z, t), G_{R}(\psi ; w, z, t)$ and $G\left(\psi, \psi^{\prime} ; w, z, t\right)$ have the properties (1)-(5) stated in Theorem 1.11 .

\section{§5. Integral Operators}

In this section we firstly study integral operators with kernels $K(w, z, t)$ $\in \kappa\left(W_{\delta}\right)$ and secondly give the proof of Theorem 1.11. Let us recall $(w, z, t) \in$ $\boldsymbol{C}^{1} \times \boldsymbol{C}^{n+1} \times \boldsymbol{C}^{N+1}, t=\left(t_{0}, t_{1}, \cdots, t_{N}\right), X=\left\{(z, t) \in \boldsymbol{C}^{n+1} \times \boldsymbol{C}^{N+1} ;|z| \leqq r_{1},\left|t_{0}\right| \leqq r_{3}, r_{2} \leqq\right.$ $\left.\left|t_{i}\right| \leqq r_{3}(1 \leqq i \leqq N)\right\}$,

$$
W_{\delta}=\left\{(w, z, t) \in C^{1} \times C^{n+1} \times C^{N+1} ;|w|>(\sin \delta)\left|t_{0}\right|,(z, t) \in X\right\},
$$

where $0<r_{1}<r_{2}<r_{3}$ and $\delta>0$ is small and put

$$
\left\{\begin{array}{l}
U=\left\{z \in C^{n+1} ;|z| \leqq r_{1}\right\}, \quad \Omega=\left\{t \in C^{N+1} ;|t| \leqq r_{3}\right\}, \\
X_{\Lambda_{0}}=X \times \tilde{\Lambda}_{0}^{*}, \quad \Lambda_{0}^{*}=\left\{\lambda \in C^{1} ;|\lambda| \geqq \Lambda_{0}\right\} .
\end{array}\right.
$$

We also write again a path $T(a, b)$ in $t$-space, $a<b$ and $b-a \leqq 2 \pi$ (see $\S 1$ ), which is defined as follows: $T(a, b)=T_{0}(a, b) \times T^{\prime} \subset C^{1} \times C^{N} . \quad T_{0}(a, b)=T_{0}^{1}(a, b)$ $+T_{0}^{2}(a, b)+T_{0}^{3}(a, b)$ is a path in $t_{0}$-space, where $T_{0}^{1}(a, b)=\left\{t_{0}=\left((1-s) r_{3}+s \eta\right) e^{\imath a}\right.$; $0 \leqq s \leqq 1\}, \quad T_{0}^{2}(a, b)=\left\{t_{0}=\eta e^{i \varphi} ; a \leqq \varphi \leqq b\right\} \quad$ and $\quad T_{0}^{3}(a, b)=\left\{t_{0}=\left(s r_{3}+(1-s) \eta\right) e^{i b}\right.$; $0 \leqq s \leqq 1\} \quad\left(0<\eta<r_{3}\right) . \quad T^{\prime}$ is the product of paths $\left|t_{i}\right|=r_{3}\left(t_{i}=r_{3} e^{i \varphi} ; 0 \leqq \varphi \leqq 2 \pi\right)$ $(i=1,2, \cdots, N)$ in $C^{N} . \quad \eta>0$ in $T_{0}(a, b)$ is chosen suitably and small in order to obtain good estimates.

Let $K(w, z, t) \in \kappa\left(W_{\delta}\right)$ and $f(t) \in \mathcal{O}(\Omega(a, b)), b-a>2 \delta$. Define

$$
(K f)(z)=\int_{T} K\left(t_{0}-z_{0}, z, t\right) f(t) d t,
$$

where $T=T\left(a^{\prime}, b^{\prime}\right)$ with $a<a^{\prime}<b^{\prime}<b$ and $2 \delta<b^{\prime}-a^{\prime} \leqq 2 \pi$. We have

Proposition 5.1. Let $K(w, z, t) \in \kappa\left(W_{\delta}\right)$ and $f(z) \in \mathcal{O}(\Omega(a, b)), \quad b-a>2 \delta$. Then $(K f)(z) \in \mathcal{O}(U(a+\delta, b-\delta))$. 
Proof. We note that $K(w, z, t)$ is holomorphic if $|w|>(\sin \delta)\left|t_{0}\right|$ as a function of $w$. So, if $z_{0}$ satisfies $\left|z_{0}-t_{0}\right|>(\sin \delta)\left|t_{0}\right|$ for $t_{0} \in T_{0}\left(a^{\prime}, b^{\prime}\right),(K f)(z)$ is holomorphic. So we obtain $(K f)(z) \in \mathcal{O}\left(U\left(a^{\prime}+\delta, b^{\prime}-\delta\right)\right)$. The integration path $T$ depends on $a^{\prime}$ and $b^{\prime}$. Let $\left(K^{\prime} f\right)(z)$ be an operator integrated on $T\left(a^{\prime}, b^{\prime}\right)$ and $\left(K^{\prime \prime} f\right)(z)$ be one integrated on $T\left(a^{\prime \prime}, b^{\prime \prime}\right)$. We can easily show that if $\left(a^{\prime}+\delta, b^{\prime}-\delta\right) \cap\left(a^{\prime \prime}+\delta, b^{\prime \prime}-\delta\right) \neq \varnothing$, then $\left(K^{\prime} f\right)(z)-\left(K^{\prime \prime} f\right)(z)$ is holomorphic in a neighbourhood of $z=0$. Thus $(K f)(z)$ is holomorphically extensible to $U(a+\delta, b-\delta)$.

Proposition 5.2. Suppose that $\hat{K}(z, t, \lambda) \in \mathcal{O}\left(X_{\Lambda_{0}}\right)$ satisfies

$$
|\hat{K}(z, t, \lambda)| \leqq A \exp \left((\sin \delta)\left|\lambda t_{0}\right|+B \mid \lambda i^{a}\right) \quad \text { on } X_{d_{0}}
$$

for $A, B, 0<\alpha<1$ and $0 \leqq \delta<\pi / 2$. Put

$$
K(w, z, t)=\int_{\Lambda_{0}}^{\infty i \varphi} \exp (-\lambda w) \hat{K}(z, t, \lambda) d \lambda .
$$

Then $K(w, z, t) \in \boldsymbol{\kappa}\left(W_{\delta}\right)$ and the following holds.

(1) Let $f(z) \in \mathcal{O}_{\{\gamma\}, h}\left(\Omega\left(-\hat{\psi}+(\pi / 2)-\varepsilon_{0},-\hat{\psi}+(3 \pi / 2)+\varepsilon_{0}\right)\right), \varepsilon_{0}>\delta, \gamma=\alpha /(1-\alpha)$. Then for any $\varepsilon^{\prime}>0$ there is an $h^{\prime}\left(\varepsilon^{\prime}\right)$ such that $(K f)(z) \in \mathcal{O}_{(\gamma), h},\left(\Omega\left(-\hat{\psi}+(\pi / 2)+\varepsilon^{\prime}\right.\right.$, $\left.\left.-\hat{\phi}+(3 \pi / 2)-\varepsilon^{\prime}\right)\right)$.

(2) Suppose that

$$
|\hat{K}(z, t, \lambda)| \leqq A \exp \left((\sin \delta)\left|\lambda t_{0}\right|-C|\lambda|^{a}\right) \quad \text { on } \arg \lambda=\hat{\psi} \text {. }
$$

Let $f(z) \in \mathcal{O}_{(\gamma), h}\left(\Omega\left(-\hat{\psi}+(\pi / 2)-\varepsilon_{0},-\hat{\psi}+(3 \pi / 2)+\varepsilon_{0}\right)\right), \varepsilon_{0}>\delta, \gamma=\alpha /(1-\alpha)$. Then there is an $h_{1}$ such that if $0<h<h_{1},(K f)(z) \in A s y_{(\gamma)}(U(-\hat{\psi}+(\pi / 2),-\hat{\psi}+(3 \pi / 2))$.

(3) Suppose that

$$
|\hat{K}(z, t, \lambda)| \leqq A(1+|\lambda|)^{N} \exp \left((\sin \delta)\left|\lambda t_{0}\right|\right) \quad \text { on } \arg \lambda=\hat{\psi} .
$$

Let $\kappa>0$ be arbitrary. If $f(z) \in \mathcal{O}_{\{\kappa\}, h}\left(\Omega\left(-\hat{\psi}+(\pi / 2)-\varepsilon_{0},-\hat{\psi}+(3 \pi / 2)+\varepsilon_{0}\right)\right), \varepsilon_{0}>\delta$, then for any $\varepsilon^{\prime}>0$ there is a constant $c=c\left(\varepsilon^{\prime}\right)>0$ such that $(K f)(z) \in \mathcal{O}_{(\kappa), c h}(\Omega(-\hat{\phi}$ $\left.\left.+(\pi / 2)+\varepsilon^{\prime},-\hat{\phi}+(3 \pi / 2)-\varepsilon^{\prime}\right)\right)$. If $f(z) \in A s y_{(\kappa)}\left(\Omega\left(-\hat{\psi}+(\pi / 2)-\varepsilon_{0},-\hat{\psi}+(3 \pi / 2)+\varepsilon_{0}\right)\right)$, $\varepsilon_{0}>\delta$, then $(K f)(z) \in A s y_{(\kappa)}(\Omega(-\hat{\psi}+(\pi / 2),-\hat{\psi}+(3 \pi / 2))$.

Proof. It is obvious that $K(w, z, t) \in \kappa\left(W_{\delta}\right)$. Put $\Psi\left(\lambda, t_{0}\right)=-\operatorname{Re} \lambda t_{0}+$ $(\sin \delta)\left|\lambda t_{0}\right|+h\left|t_{0}\right|^{-\gamma}+B|\lambda|^{\alpha}, \delta^{\prime}=\left(\delta+\varepsilon_{0}\right) / 2$ and $T=T\left(-\hat{\phi}+(\pi / 2)-\delta^{\prime},-\hat{\phi}+(3 \pi / 2)\right.$ $\left.\left.+\delta^{\prime}\right)\right)$. Let $\arg \lambda=\hat{\psi}$. Put $\eta=c|\lambda|^{-1+a}$ in the definition of $T$. Then we have $\Psi\left(\lambda, t_{0}\right)=-c_{1}\left|\lambda t_{0}\right|+h\left|t_{0}\right|^{-\gamma}+B|\lambda|^{\alpha}$ on $T_{0}^{1}\left(-\hat{\psi}+(\pi / 2)-\delta^{\prime},-\hat{\psi}+(3 \pi / 2)+\delta^{\prime}\right), c_{1}=$ $\sin \delta^{\prime}-\sin \delta$. So $\Psi\left(\lambda, t_{0}\right) \leqq\left(B+h c^{-r}\right)|\lambda|^{a}$ on $T_{0}^{1}\left(-\hat{\phi}+(\pi / 2)-\delta^{\prime},-\hat{\phi}+(3 \pi / 2)+\delta^{\prime}\right)$. We have also, $\Psi\left(\lambda, t_{0}\right) \leqq\left(B+h c^{-\gamma}\right)|\lambda|^{n}$ on $T_{0}^{3}\left(-\phi+(\pi / 2)-\delta^{\prime},-\hat{\phi}+(3 \pi / 2)+\delta^{\prime}\right)$. On $T_{0}^{2}\left(-\hat{\psi}+(\pi / 2)-\delta^{\prime},-\hat{\psi}+(3 \pi / 2)+\delta^{\prime}\right), \Psi\left(\lambda, t_{0}\right) \leqq\left(c+c \sin \delta+h c^{-\gamma}+B\right)|\lambda|^{a}$. Hence we have 


$$
\Psi\left(\lambda, t_{0}\right) \leqq\left(c+c \sin \delta+h c^{-\gamma}+B\right)|\lambda|^{\alpha} \quad \text { on } T_{0}\left(-\hat{\psi}+\frac{\pi}{2}-\delta^{\prime},-\hat{\psi}+\frac{3 \pi}{2}+\delta^{\prime}\right) .
$$

So we have, if $\arg \lambda=\hat{\phi}$,

$$
\left|\int_{T} \exp \left(-\lambda t_{0}\right) \hat{K}(z, t, \lambda) f(t) d t\right| \leqq A \exp \left(\left(c+c \sin \delta+h c^{-r}+B\right)|\lambda|^{a}\right)
$$

for any $c>0$. Thus we have, if $\left|\arg z_{0}+\hat{\phi}-\pi\right|<(\pi / 2)-\varepsilon^{\prime}$,

$$
(K f)(z)=\int_{1_{0}}^{\infty e^{i \varphi}} \exp \left(\lambda z_{0}\right) d \lambda \int_{T} \exp \left(-\lambda t_{0}\right) \hat{K}(z, t, \lambda) f(t) d t
$$

and from (5.9)

$$
\begin{aligned}
|(K f)(z)| & \leqq A\left|\int_{A_{0}}^{+\infty} \exp \left(-\left(\sin \varepsilon^{\prime}\right)\left|z_{0}\right| r+\left(c+c \sin \delta+h c^{-r}+B\right) r^{a}\right) d r\right| \\
& \leqq A\left|z_{0}\right|^{-N-1} \exp \left(h^{\prime}\left|z_{0}\right|^{-r}\right) .
\end{aligned}
$$

We have (1). Let us show (2). Assume (5.6). Put $\Psi\left(\lambda, t_{0}\right)=-\operatorname{Re} \lambda t_{0}+(\sin \delta)\left|\lambda t_{0}\right|$ $\left.+h\left|t_{0}\right|^{-r}, T=T\left(-\hat{\psi}+(\pi / 2)-\delta^{\prime},-\hat{\psi}+(3 \pi / 2)+\delta^{\prime}\right)\right), \delta^{\prime}=\left(\delta+\varepsilon_{0}\right) / 2$ and let $\arg \lambda=\hat{\psi}$. Put $\eta=c|\lambda|^{-1+\alpha}$ in the definition of $T$. Then we have

$$
\Psi\left(\lambda, t_{0}\right) \leqq\left(c+c \sin \delta+h c^{-r}\right)|\lambda|^{a} \quad \text { on } T_{0}\left(-\hat{\psi}+\frac{\pi}{2}-\delta^{\prime},-\hat{\phi}+\frac{3 \pi}{2}+\delta^{\prime}\right) .
$$

So we have, if $\arg \lambda=\hat{\phi}$,

$$
\left|\int_{T} \exp \left(-\lambda t_{0}\right) \hat{K}(z, t, \lambda) f(t) d t\right| \leqq A \exp \left(\left(c+c \sin \delta+h c^{-r}-C\right)|\lambda|^{a}\right)
$$

for any $c>0$ and if $\left|\arg z_{0}+\hat{\phi}-\pi\right|<\pi / 2$,

$$
|(K f)(z)| \leqq A\left|\int_{\Lambda_{0}}^{+\infty} \exp \left(\left(c+c \sin \delta+h c^{-\gamma}-C\right) r^{a}\right) d r\right| .
$$

Choose $c>0$ small so that $c+c \sin \delta \leqq 2 c \leqq C / 4$, fix it and choose $h_{1}$ with $h_{1} c^{-\gamma}$ $\leqq C / 4$. Hence if $0<h<h_{1}$, for $\left|\arg z_{0}+\hat{\psi}-\pi\right|<\pi / 2$

$$
|(K f)(z)| \leqq A\left|\int_{\Lambda_{0}}^{\infty} \exp \left(-C r^{\alpha} / 2\right) d r\right| .
$$

We also have

$$
\left|\partial_{0}^{r}(K f)(z)\right| \leqq A \sum_{s=0}^{r}\left|\int_{\Lambda_{0}}^{\infty i \hat{\psi}} \frac{r !}{s !} \exp \left(-C|\lambda|^{a} / 2\right)(1+|\lambda|)^{s} d \lambda\right| \leqq A B^{r} \Gamma\left(\frac{r}{\alpha}+1\right) .
$$

This means that if $0<h<h_{1},(K f)(z) \in A s y_{(\gamma)}(U(-\hat{\phi}+(\pi / 2),-\hat{\phi}+(3 \pi / 2))$. Let us show (3). Assume (5.7). Let $f(z) \in \mathcal{O}_{(\kappa), h}\left(\Omega\left(-\hat{\psi}+(\pi / 2)-\varepsilon_{0},-\hat{\psi}+(3 \pi / 2)+\varepsilon_{0}\right)\right)$, $T=\left(-\hat{\psi}+(\pi / 2)-\delta^{\prime},-\hat{\phi}+(3 \pi / 2)+\delta^{\prime}\right), \delta^{\prime}=\left(\delta+\varepsilon_{0}\right) / 2$, and $\arg \lambda=\hat{\psi}$. We have in the same way as in (1), for any $c>0$

$$
\left|\int_{T} \exp \left(-\lambda t_{0}\right) \hat{K}(z, t, \lambda) f(t) d t\right| \leqq A(1+|\lambda|)^{N} \exp \left(\left(c+c \sin \delta+h c^{-\kappa}\right)|\lambda|^{\alpha}\right),
$$


$\hat{\alpha}=\kappa /(1+\kappa)$. Hence we have for $-\hat{\phi}+(\pi / 2)<\arg z_{0}<-\hat{\phi}+(3 \pi / 2)$,

$$
(K f)(z)=\int_{\Lambda_{0}}^{\infty i \hat{\psi}} \exp \left(\lambda z_{0}\right) d \lambda \int_{T} \exp \left(-\lambda t_{0}\right) \hat{K}(z, t, \lambda) f(t) d t
$$

and

$$
|(K f)(z)| \leqq A\left|\int_{\Lambda_{0}}^{\infty i \hat{\psi}} \exp \left(\operatorname{Re} \lambda z_{0}+\left(c+c \sin \delta+h c^{-\kappa}\right)|\lambda|^{\hat{\alpha}}\right)(1+|\lambda|)^{N} d \lambda\right|
$$

Choose $c=h^{1 /(\kappa+1)}$, then $c+c \sin \delta+h c^{-\kappa}=\hat{c} h^{1-\hat{\alpha}}$ for a $\hat{c}>0$ independent of $h$. Thus we have for $-\phi+(\pi / 2)+\varepsilon^{\prime}<\arg z_{0}<-\phi+(3 \pi / 2)-\varepsilon^{\prime}$

$$
\begin{aligned}
|(K f)(z)| & \leqq A\left|\int_{\Lambda_{0}}^{+\infty} \exp \left(-\sin \varepsilon^{\prime}\left|z_{0}\right| r+\hat{c} h^{1-\hat{a}} r^{\hat{\alpha}}\right)(1+r)^{N} d r\right| \\
& \leqq A\left|z_{0}\right|^{-N-1} \exp \left(c_{1} h\left|z_{0}\right|^{-\kappa}\right) \leqq A \exp \left(c h\left|z_{0}\right|^{-\kappa}\right), \quad c=c\left(\varepsilon^{\prime}\right)
\end{aligned}
$$

This means the first statement of (3). Let us show the second statement of (3). Let $f(z) \in A s y_{(\kappa)} \Omega\left(-\hat{\psi}+(\pi / 2)-\varepsilon_{0},-\hat{\psi}+(3 \pi / 2)+\varepsilon_{0}\right)$. Then we have

$$
\begin{aligned}
& \int_{T} \exp \left(-\lambda t_{0}\right) \hat{K}(z, t, \lambda) f(t) d t \\
& =\int_{T} \exp \left(-\lambda t_{0}\right) \hat{K}(z, t, \lambda)\left\{\sum_{k=0}^{q} t_{0}^{k}\left(\partial_{t_{0}}\right)^{k} f\left(0, t^{\prime}\right) / k !+t_{0}^{q+1} \tilde{f}(t) /(q+1) !\right\} d t .
\end{aligned}
$$

Put

$$
g_{q}(z, \lambda)=\int_{T} \exp \left(-\lambda t_{0}\right) \hat{K}(z, t, \lambda)\left\{\sum_{k=0}^{q} t_{0}{ }^{k}\left(\partial_{t_{0}}\right)^{k} f\left(0, t^{\prime}\right) / k !\right\}
$$

and

$$
h_{q}(z, \lambda)=\int_{T} \exp \left(-\lambda t_{0}\right) \hat{K}(z, t, \lambda) t_{0}^{q+1} \tilde{f}(t) /(q+1) ! d t .
$$

By deforming the path $T$ and putting $c_{2}=\left(\sin \delta^{\prime}-\sin \delta\right) r_{3}>0$, we have

$$
\begin{aligned}
& \left|g_{q}(z, \lambda)\right| \leqq A|\lambda|^{N} \exp \left(-c_{2}|\lambda|\right)\left(\sum_{k=0}^{q} A B^{k} \Gamma\left(\frac{k}{\kappa}+1\right)\right) \\
\leqq & A|\lambda|^{N} \exp \left(-c_{2}|\lambda|\right) B^{q} \Gamma\left(\frac{q+1}{\kappa}+1\right) \leqq A|\lambda|^{N-q} B^{q} q ! \Gamma\left(\frac{q+1}{\kappa}+1\right) \text { for } \arg \lambda=\hat{\phi},
\end{aligned}
$$

and

$$
\begin{aligned}
\left|h_{q}(z, \lambda)\right| & \leqq \int_{T}\left|\exp \left(-\lambda t_{0}\right) \hat{K}(z, t, \lambda) t_{0}^{q+1} \dot{f}(t) /(q+1) !\right| d t \\
& \leqq A|\lambda|^{N} \int_{T} \exp \left(-c_{2}\left|\lambda t_{0}\right|\right)\left|B t_{0}\right|^{q+1} \Gamma\left(\frac{q+1}{\kappa}+1\right) d t \\
& \leqq A|\lambda|^{N-q-1} \int_{T} \exp \left(-c_{2}\left|\lambda t_{0}\right|\right)\left|B \lambda t_{0}\right|^{q+1} \Gamma\left(\frac{q+1}{\kappa}+1\right) d t
\end{aligned}
$$




$$
\leqq A|\lambda|^{N-q-1} C\left(\delta^{\prime}, \delta\right)^{q+1} \Gamma\left(\frac{q+1}{\kappa}+1\right)(q+1) ! \quad \text { for } \arg \lambda=\hat{\psi} .
$$

Therefore we have for $\arg \lambda=\hat{\psi}$

$$
\left|\int_{T} \exp \left(-\lambda t_{0}\right) \hat{K}(z, t, \lambda) f(t) d t\right| \leqq A \mid \lambda i^{N-q-1} B\left(\delta^{\prime}, \delta\right)^{q+1} \Gamma\left(\frac{q+1}{\kappa}+1\right) q ! .
$$

Since the estimate (5.20) is valid for large $\lambda$ and any $q \in N$,

$$
\left|\int_{T} \exp \left(-\lambda t_{0}\right) \hat{K}(z, t, \lambda) f(t) d t\right| \leqq A(\varepsilon, \delta) \exp \left(-c|\lambda|^{\hat{\alpha}}\right), \quad \hat{\alpha}=\frac{\kappa}{\kappa+1} .
$$

Hence we have if $\left|\arg z_{0}+\hat{\phi}-\pi\right|<\pi / 2$,

$$
(K f)(z)=\int_{\Lambda_{0}}^{\infty e^{i \varphi}} \exp \left(\lambda z_{0}\right) d \lambda \int_{T} \exp \left(-\lambda t_{0}\right) \hat{K}(z, t, \lambda) f(t) d t
$$

and from (5.21)

$$
\left|\partial_{0}^{r}(K f)(z)\right| \leqq A B^{r} \sum_{s=0}^{r}\left|\int_{\Lambda_{0}}^{\infty e^{2 \varphi}} \frac{r !}{s !} \exp \left(-c|\lambda|^{\hat{a}}\right)(1+|\lambda|)^{s} d \lambda\right| \leqq A B^{r} \Gamma\left(\frac{r}{\hat{\alpha}}+1\right) .
$$

This means that $(K f)(z) \in A s y_{(\kappa)}(U(-\hat{\psi}+(\pi / 2),-\hat{\psi}+(3 \pi / 2)))$.

Now we apply Propositions 5.1 and 5.2 to the integral operators defined by (5.3) with kernels $K(w, z, t)=G(\psi ; w, z, t), G_{R}(\psi ; w, z, t)$ or $G\left(\psi^{\prime}, \psi^{\prime \prime} ; u^{\prime}, z, t\right)$ (see (4.13), (4.14) and (4.21)). They are denoted by $\left(G^{\psi} f\right)(z),\left(G_{R}^{\psi} f\right)(z)$ or $\left(G^{\dot{\prime}^{\prime}, \psi^{\prime \prime}} f\right)(z)$ respectively. In the following considerations

$$
\left\{\begin{array}{l}
\alpha=\alpha_{p-1}=\left(\sigma_{p-1}-1\right) / \sigma_{p-1}, \text { and } \gamma=\sigma_{p-1}-1=\alpha_{p-1} /\left(1-\alpha_{p-1}\right), \\
\delta^{*}=\sin ^{-1} C^{*} d^{*} \quad \text { (see Proposition 4.4). }
\end{array}\right.
$$

From Lemma 4.3 and (4.22) we have the estimates:

$$
|\hat{V}(\psi ; z, t, \lambda)| \leqq A|\lambda|^{q^{*}} \exp \left(\left(\sin \delta^{*}\right)\left|\lambda t_{0}\right|+d^{*}|\lambda|^{\alpha}\right),
$$

(5.26) $|\hat{V}(\phi ; z, t, \lambda)| \leqq A|\lambda|^{q^{*}} \exp \left(\left(\sin \delta^{*}\right)\left|\lambda t_{0}\right|\right) \quad$ for $|\arg \lambda+\phi|<\pi / 2 \alpha$,

$$
\begin{aligned}
& \left|\hat{V}\left(\psi^{\prime}, \psi^{\prime \prime} ; z, t, \lambda\right)\right| \leqq A|\lambda|^{q^{*}} \exp \left(\left(\sin \delta^{*}\right)\left|\lambda t_{0}\right|-d^{*} \sin (\alpha \varepsilon)|\lambda|^{a}\right) \\
& \quad \text { for }-\frac{\pi}{2 \alpha}+\frac{\left|\phi^{\prime \prime}-\psi^{\prime}\right|}{2}+\varepsilon<\arg \lambda+\frac{\phi^{\prime \prime}+\psi^{\prime}}{2}<\frac{\pi}{2 \alpha}-\frac{\left|\psi^{\prime \prime}-\psi^{\prime}\right|}{2}-\varepsilon \quad \text { with } \\
& \quad 0<\alpha \varepsilon<\frac{\pi}{2}-\frac{\alpha\left|\psi^{\prime \prime}-\psi^{\prime}\right|}{2}
\end{aligned}
$$

and

(5.28) $\left.\mid \exp \left(-\lambda^{\alpha} d^{*} e^{\imath \alpha \psi}\right)\right) V_{R}(\phi ; z, t, \lambda) \mid$

$$
\leqq A(1+|\lambda|)^{N} \exp \left(\left(\sin \delta^{*}\right)\left|\lambda t_{0}\right|-d^{*} \sin (\alpha \varepsilon)|\lambda|^{a}\right) \text { for }|\arg \lambda+\phi|<\frac{\pi}{2 \alpha}-\varepsilon .
$$

Proposition 5.3. Let $f(z) \in \mathcal{O}_{(\gamma), h}(\Omega(a, b))$, where $\phi-\pi / 2 \alpha+\pi / 2<a<b<\phi+$ 
$\pi / 2 \alpha+3 \pi / 2, b-a>\pi+2 \delta^{*}$. Then for any $\varepsilon>\delta^{*}$ there is a constant $c=c(\varepsilon)>0$ such that $\left(G^{\psi} f\right)(z) \in \mathcal{O}_{(r), c h}(U(a+\varepsilon, b-\varepsilon))$.

Proof. Put $\hat{K}(z, t, \lambda)=\hat{V}(\phi ; z, t, \lambda)$ in Proposition 5.2. Choose $\varepsilon_{0}$ so that $b-a>\pi+2 \varepsilon_{0}>\pi+2 \delta^{*}$ and $\varepsilon>\varepsilon_{0}$. Then it follows from the assumption on $a$ and $b$ that $\hat{\psi}$ with $a-(\pi / 2)+\varepsilon_{0}<-\hat{\psi}<b-(3 \pi / 2)-\varepsilon_{0}$ satisfies $|\hat{\psi}+\phi|<(\pi / 2 \alpha)-\varepsilon_{0}$ and (5.26) holds. Hence it follows from Proposition 5.2-(3) that for any $\varepsilon^{\prime}>0$ there is a $c=c\left(\varepsilon^{\prime}\right)>0$ such that $\left(G^{\dot{\psi}} f\right)(z) \in \mathcal{O}_{(\gamma), c h}\left(U\left(-\hat{\phi}+\pi / 2+\varepsilon^{\prime},-\hat{\phi}+3 \pi / 2-\varepsilon^{\prime}\right)\right)$. The union of intervals $\left(-\hat{\phi}+(\pi / 2)+\varepsilon^{\prime},-\hat{\phi}+(3 \pi / 2)-\varepsilon^{\prime}\right), a-(\pi / 2)+\varepsilon_{0}<-\hat{\phi}<b-$ $(3 \pi / 2)-\varepsilon_{0}$, is $\left(a+\varepsilon_{0}+\varepsilon^{\prime}, b-\varepsilon_{0}-\varepsilon^{\prime}\right)$. By putting $\varepsilon^{\prime}=\varepsilon-\varepsilon_{0}$, we have the assertion.

Proposition 5.4. Suppose $\phi^{\prime}<\psi^{\prime \prime}, 0<\alpha \delta^{*}<\left(\pi-\alpha_{\mid}\left|\psi^{\prime \prime}-\psi^{\prime}\right|\right) / 2$. Let $f(z) \in$ $\mathcal{O}_{(r), h}(\Omega(a, b))$, where $\phi^{\prime \prime}-(\pi / 2 \alpha)+(\pi / 2)<a<b<\psi^{\prime}+(\pi / 2 \alpha)+(3 \pi / 2)$ and $b-a>\pi$ $+2 \delta^{*}$. Then there is an $h_{0}$ such that if $0<h<h_{0},\left(G^{\dot{\varphi}^{\prime}, \dot{\psi}^{\prime \prime}} f\right)(z) \subseteq A s y_{(r)}\left(\Omega\left(a+\delta^{*}\right.\right.$, $\left.\left.b-\delta^{*}\right)\right)$.

Proof. Put $\hat{K}(z, t, \lambda)=V\left(\psi^{\prime}, \psi^{\prime \prime} ; z, t, \lambda\right)$ in Proposition 5.2. Choose $\varepsilon_{0}$ so that $b-a>\pi+2 \varepsilon_{0}>\pi+2 \delta^{*}$ and $\alpha \varepsilon_{0}<\left(\pi-\alpha\left|\psi^{\prime \prime}-\psi^{\prime}\right|\right) / 2$. Then it follows from the assumption on $a$ and $b$ that $\hat{\psi}$ with $a-(\pi / 2)+\varepsilon_{0}<-\hat{\psi}<b-(3 \pi / 2)-\varepsilon_{0}$ satisfies $-(\pi / 2) \alpha+\left(\psi^{\prime \prime}-\psi^{\prime}\right) / 2+\varepsilon_{0}<\hat{\psi}+\left(\psi^{\prime}+\psi^{\prime \prime}\right) / 2<(\pi / 2 \alpha)-\left(\psi^{\prime \prime}-\psi^{\prime}\right) / 2-\varepsilon_{0}$ and (5.27) holds for $\arg \lambda=\hat{\phi}$ and $\varepsilon=\varepsilon_{0}$, where $\sin \left(\alpha \varepsilon_{0}\right) \geqq \sin \left(\alpha \delta^{*}\right)$. Intervals $(-\hat{\psi}+(\pi / 2),-\hat{\phi}+$ $(3 \pi / 2)), a-(\pi / 2)+\varepsilon_{0}<-\hat{\psi}<b-(3 \pi / 2)-\varepsilon_{0}$, cover $\left(a+\varepsilon_{0}, b-\varepsilon_{0}\right)$. We have the assertion by Proposition $5.2-(2)$, tending $\varepsilon_{0}$ to $\delta^{*}$.

Proposition 5.5. Let $f(z) \in \mathcal{O}_{(\gamma), h}(\Omega(a, b))$, where $\phi-\pi / 2 \alpha+\pi / 2<a<b<\phi+$ $\pi / 2 \alpha+3 \pi / 2, b-a>\pi+2 \delta^{*}$. Then there is an $h_{1}\left(\delta^{*}\right)>0$ such that, if $0<h<h_{1}$, $\left(G_{R}^{\psi} f\right)(z) \in A s y_{(\gamma)}\left(U\left(a+\delta^{*}, b-\delta^{*}\right)\right)$.

Proof. Put $\left.\hat{K}(z, t, \lambda)=\exp \left(-\lambda^{a} d^{*} e^{i \alpha \dot{\varphi}}\right)\right) V_{R}(z, t, \lambda)$ in Proposition 5.2. Choose $\varepsilon_{0}$ so that $b-a>\pi+2 \varepsilon_{0}>\pi+2 \delta^{*}$. Then it follows from the assumption on $a$ and $b$ that $\hat{\psi}$ with $a-(\pi / 2)+\varepsilon_{0}<-\hat{\phi}<b-(3 \pi / 2)-\varepsilon_{0}$ satisfies $|\hat{\phi}+\phi|<(\pi / 2 \alpha)-\varepsilon_{0}$ and (5.28) holds for $\varepsilon=\varepsilon_{0}$. Intervals $(-\hat{\psi}+(\pi / 2),-\hat{\psi}+(3 \pi / 2)), a-(\pi / 2)+\varepsilon_{0}<$ $-\hat{\phi}<b-(3 \pi / 2)-\varepsilon_{0}$, cover $\left(a+\varepsilon_{0}, b-\varepsilon_{0}\right)$. By tending $\varepsilon_{0}$ to $\delta^{*}$, since $\sin \left(\alpha \varepsilon_{0}\right) \geqq$ $\sin \left(\alpha \delta^{*}\right)$, we have the assertion from Proposition 5.2-(2).

Now let $\delta_{0}>0$ be a given small number. We choose $d^{*}=d^{*}\left(\delta_{0}\right)>0$ so that $c^{*} \sin \delta_{0}=C^{*} d^{*}$, by using the constants $0<c^{*} \leqq 1$ and $C^{*}>0$ in Proposition 4.4, and fix $d^{*}$. It is obvious that $\delta_{0} \geqq \delta^{*}=\sin ^{-1} C^{*} d^{*}$. In the rest of this section we consider $G(\psi ; w, z, t), G_{R}(\psi ; w, z, t)$ and $G\left(\psi^{\prime}, \phi^{\prime \prime} ; w, z, t\right)$ for this fixed $d^{*}=d^{*}\left(\delta_{0}\right)$. By Proposition 4.4, if $|\arg w-\psi|<\pi$, and $\left(\sin \delta_{0}\right)\left|t_{0}\right|<|w|$,

$$
\left|\partial_{w}^{q} \partial_{2}^{r} G(\psi ; w, z, t)\right| \leqq \frac{A B^{q} C^{r} q ! r !}{\left(c^{*}\left(|w|-\sin \delta_{0} \mid t_{\mid}^{\prime}\right)\right)^{q^{\prime}+1^{\prime}}}, \quad q^{\prime}=q+q^{*},
$$

holds. 
Proposition 5.6. Let $f(z) \in A s y_{(\kappa)}(\Omega(a, b))$, where $\kappa>0$ is arbitrary, $\phi<a<$ $b<\psi+2 \pi$ and $b-a>2 \delta_{0}$. Then $\left(G^{\psi} f\right)(z) \in A s y_{(\kappa)}\left(U\left(a+\delta_{0}, b-\delta_{0}\right)\right)$.

Proof. We may assume $\phi=-\pi$. Let $a<a^{\prime \prime}<a^{\prime}<b^{\prime}<b^{\prime \prime}<b$ with $b^{\prime \prime}-a^{\prime \prime}>$ $b^{\prime}-a^{\prime}>2 \delta_{0}$ and $z \in U\left(a^{\prime}+\delta_{0}, b^{\prime}-\delta_{0}\right)$. We have

$$
\begin{aligned}
& \left(\partial_{z_{0}}\right)^{q}\left(G^{-\pi} f\right)(z)=\int_{T}\left(-\partial_{w}+\partial_{z_{0}}\right)^{q} G\left(-\pi ; t_{0}-z_{0}, z, t\right) f(t) d t \\
& =\int_{T} G^{(q)}\left(-\pi ; t_{0}-z_{0}, z, t\right)\left\{\sum_{k^{\prime}=0}^{q^{\prime}} t_{0}{ }^{k}\left(\partial_{t_{0}}\right)^{k} f\left(0, t^{\prime}\right) / k !+t_{0}^{q^{\prime}+1} \tilde{f}(t) /\left(q^{\prime}+1\right) !\right\} d t,
\end{aligned}
$$

where $T=T\left(a^{\prime \prime}, b^{\prime \prime}\right), G^{(q)}(-\pi ; w, z, t)=\left(-\partial_{w}+\partial_{z_{0}}\right)^{q} G(-\pi ; w, z, t)$ and $q^{\prime}=q+q^{*}$. Put

$$
g_{q}(z)=\int_{T} G^{(q)}\left(-\pi ; t_{0}-z_{0}, z, t\right)\left\{\sum_{k=0}^{q^{\prime}} t_{0}^{k}\left(\partial_{t_{0}}\right)^{k} f\left(0, t^{\prime}\right) / k !\right\} d t
$$

and

$$
h_{q}(z)=\int_{T} G^{(q)}\left(-\pi ; t_{0}-z_{0}, z, t\right) t_{0}^{q^{\prime}+1} \tilde{f}(t) /\left(q^{\prime}+1\right) ! d t .
$$

By deforming the path $T$, we have $g_{q}(z) \in \mathcal{O}(U)$ and $\left|g_{q}(z)\right| \leqq q ! \sum_{k=0}^{q^{\prime}} A B^{q} C^{k} \Gamma$ $\times((k / \kappa)+1) \leqq A B^{q} D q ! \Gamma\left(\left(q^{\prime}+1\right) / \kappa+1\right)$. Since $-\pi<a<a^{\prime \prime}<a^{\prime}<b^{\prime}<b^{\prime \prime}<b<\pi$ and $b^{\prime \prime}-a^{\prime \prime}>b^{\prime}-a^{\prime}>2 \delta_{0}$, there is a $\delta_{1}>\delta_{0}$ such that $\sin \delta_{1}\left|t_{0}\right|<\left|z_{0}-t_{0}\right|$ for $t \in T=$ $T\left(a^{\prime \prime}, b^{\prime \prime}\right)$ and $z \in U\left(a^{\prime}+\delta_{0}, b^{\prime}-\delta_{0}\right)$, and since $t \in T=T\left(a^{\prime \prime}, b^{\prime \prime}\right) \subset \Omega(-\pi, \pi)$ and $z \in U\left(a^{\prime}+\delta_{0}, b^{\prime}-\delta_{0}\right) \subset U(-\pi, \pi),\left|\arg \left(t_{0}-z_{0}\right)-\pi\right|<\pi$. Therefore by (5.29)

$$
\begin{aligned}
\left|h_{q}(z)\right| & \leqq A q ! \int_{T} \frac{B^{q}\left|t_{0}\right|^{q^{\prime}+1} \Gamma\left(\frac{q^{\prime}+1}{\kappa}+1\right)}{\left\{\left(\left|t_{0}-z_{0}\right|-\sin \delta_{0}\left|t_{0}\right|\right)\right\}^{q^{\prime}+1}}\left|d t_{0}\right| . \\
& \leqq A q ! \int_{T} \frac{B^{q}\left|t_{0}\right|^{q^{\prime+1}} \Gamma\left(\frac{q^{\prime}+1}{\kappa}+1\right)}{\left\{\left(\sin \delta_{1}-\sin \delta_{0}\right)\left|t_{0}\right|\right\}^{q^{\prime}+1}}\left|d t_{0}\right| \leqq A q ! B^{q} \Gamma\left(\frac{q^{\prime}+1}{\kappa}+1\right) .
\end{aligned}
$$

Thus we have

$$
\left|\left(\partial_{z_{0}}\right)^{q}\left(G^{-\pi} f\right)(z)\right| \leqq\left|g_{q}(z)\right|+\left|h_{q}(z)\right| \leqq A B^{q} q ! \Gamma\left(\frac{q^{\prime}+1}{\kappa}+1\right)
$$

for $z \in U\left(a^{\prime}+\delta_{0}, b^{\prime}-\delta\right)$. This means $\left(G^{-\pi} f\right)(z)$ has the $\kappa$-asymptotic expansion in $U\left(a^{\prime}+\delta_{0}, b^{\prime}-\delta_{0}\right)$. Since $a^{\prime}$ and $b^{\prime}$ are arbitraty, $\left(G^{-\pi} f\right)(z)$ has the $\kappa$-asymptotic expansion in $U\left(a+\delta_{0}, b-\delta_{0}\right)$.

Now we show (1)-(5) in Theorem 1.11.

Proof of Theorem 1.11. It follows from Proposition 4.5 that 


$$
\begin{aligned}
L\left(z, \partial_{z}\right)\left(G^{\dot{\psi}} f\right)(z) & =\frac{-1}{(2 \pi i)^{n+1}} \int_{T} \Pi_{i=0}^{n}\left(t_{i}-z_{i}\right)^{-1} f(t) d t+\left(G_{R}^{\psi} f\right)(z) \\
& =f(z)+G_{R}^{\psi}(z)+\frac{-1}{(2 \pi i)^{n+1}} \int_{T c} \Pi_{i=0}^{n}\left(t_{i}-z_{i}\right)^{-1} f(t) d t,
\end{aligned}
$$

where $T^{c}$ is a path $T_{0}^{c} \times T^{\prime}, T_{0}^{c}=T_{0}^{c}(a, b)=\left\{t_{0}=r_{3} e^{i((1-s) b+s a)} ; 0 \leqq s \leqq 1\right\}$. The last term integrated on $T^{c}$ is holomorphic at $z=0$. So we have (1) in Theorem 1.11. We proceed to the proof of (2)-(4). Let $f(z) \in \mathcal{O}(\Omega(a, b))$. We have (2) in Theorem 1.11 by Proposition 5.3, (3) by Proposition 5.4 and (4) by Proposition 5.6. Finally we show (5). If $b-a>\pi+2 \delta_{0}$, if follows from Proposition 5.5. Otherwise, by Proposition 2.12, we have for given $h^{\prime}$ with $h_{1}>h^{\prime}>h, h_{1}$ being that in Proposition 5.5, $f(z)=f_{1}(z)+f_{2}(z)$, where $f_{1}(z) \in \mathcal{O}_{(\gamma), h},(\Omega(a-2 \pi, b))$, $f_{2}(z) \in \mathcal{O}_{(\gamma), h},(\Omega(a, b+2 \pi))$. We have, from the assumptions on $a$ and $b, \psi-$ $\pi / 2 \alpha+\pi / 2<a-\pi<b+\pi<\phi+\pi / 2 \alpha+3 \pi / 2$. Hence by Proposition 5.5, $\left(G_{R}^{\psi} f_{1}\right)(z) \in$ $A s y_{(\gamma)}\left(U\left(a-\pi+\delta_{0}, b-\delta_{0}\right)\right)$ and $\left(G_{R}^{\psi} f_{2}\right)(z) \in A s y_{(\eta)}\left(U\left(a+\delta_{0}, b+\pi-\delta_{0}\right)\right)$. Since $\left(G_{R}^{\psi} f\right)$ $\equiv\left(G_{R}^{\psi} f_{1}\right)(z)+\left(G_{R}^{\psi} f_{2}\right)(z),(\bmod \mathcal{O}(U)),\left(G_{R}^{\psi} f\right)(z) \in A s y_{(r)}\left(U\left(a+\delta_{0}, b-\delta_{0}\right)\right)$.

\section{§ 6. Integral Representation}

In $\S 6$ we obtain an integal representation of a solution $u(z) \in \mathcal{O}\left(\Omega\left(\theta_{0}\right)\right)$ of

$$
L\left(z, \partial_{z}\right) u(z)=f(z),
$$

where $f(z) \in \mathcal{O}\left(\Omega\left(\theta_{0}\right)\right)$. When $f(z) \in \mathcal{O}(\Omega)$, an integral representation was obtained in [6], [7] and [12]. In this section we assume (1.11)-(a), (b), (c), namely,

(a) $\sigma_{1}>1$,

(b) $d_{k_{p-1}}=0$,

(c) $d_{k_{i}}=s_{k_{i}}$ for $0 \leqq \imath \leqq p-2$.

So we have

$$
L\left(z, \partial_{z}\right)=\sum_{k=0}^{m} \sum_{l=s_{k}}^{k} A_{k, l}\left(z, \partial^{\prime}\right) \partial_{0}^{k-l},
$$

where $d_{k_{i}}=s_{k_{\imath}}(0 \leqq \imath \leqq p-1)$, that is, $l_{k_{i}}=s_{k_{\imath}}, j\left(k_{\imath}, l_{k_{i}}\right)=0$ and $A_{k_{i}, s_{k_{i}}}\left(z, \partial^{\prime}\right)=$ $a_{k_{i}, s_{k_{i}}}\left(z, \partial^{\prime}\right)$ and by $(6.2)-(\mathrm{b}) d_{k_{p-1}}=s_{k_{p-1}}=0$. Put $\xi^{\prime}=\hat{\bar{\xi}}^{\prime}=(1,0, \cdots, 0)$. Firstly we assume

$$
\left\{\begin{array}{l}
A_{m, s_{m}}\left(0, \hat{\xi}^{\prime}\right)=a_{m, s_{m}}\left(0, \hat{\xi}^{\prime}\right) \neq 0, \\
\operatorname{II}_{i=1}^{p-1} A_{k_{i}, s_{k_{i}}}\left(0, \hat{\xi}^{\prime}\right)=\prod_{i=1}^{p-1} a_{k_{i}, s_{k_{i}}}\left(0, \hat{\xi}^{\prime}\right) \neq 0 \quad \text { (see (1.14)). }
\end{array}\right.
$$

We construct an integral representation and investigate it under the conditions (6.4). An integral representation can be constructed under the condition on the principal part in (6.4), and other conditions on lower order terms are used for the proof of Theorem 6.28 which requires detailed analysis of the integral representation. 
Before construction, we give a remark on the coordinate $z$. From (6.2) and (6.4) the principal part of $L\left(z, \partial_{z}\right)$ is written in the form

$$
L_{m}\left(z, \partial_{z}\right)=\sum_{l=s_{m}}^{m} A_{m, l}\left(z, \partial^{\prime}\right) \partial_{0}^{m-l}, \quad A_{m, s_{m}}\left(0, \hat{\xi}^{\prime}\right) \neq 0 .
$$

Consider the coordinate transformation

$$
w_{0}=z_{0}, \quad w_{1}=c z_{0}+z_{1}, \quad w_{i}=z_{i} \quad(\imath \geqq 2) .
$$

Then we have

$$
L_{m}\left(z, \partial_{z}\right)=\sum_{l=s_{m}}^{m} A_{m, l}\left(z(w), \partial_{w}^{\prime}\right)\left(\partial_{w_{0}}+c \partial_{w_{1}}\right)^{m-l} .
$$

The coefficient of $\left(\partial_{w_{1}}\right)^{m}$ is $\left(\sum_{l=s_{m}}^{m} c^{m-l} A_{m, l}\left(0, \hat{\xi}^{\prime}\right)\right)$ at the origin. Since $A_{m, s_{m}}\left(0, \hat{\xi}^{\prime}\right) \neq 0$, the coefficient of $\left(\partial_{w_{1}}\right)^{m}$ does not vanish for large $c$. This means $w_{1}=0$ is non characteristic. Hence in addition to (6.3)-(6.4) we assume that the coordinate is chosen so that

$$
A_{m, m}\left(0, \hat{\xi}^{\prime}\right) \neq 0,
$$

that is, $z_{1}=0$ is non characteristic.

The integral representation obtained here has the form

$$
\begin{aligned}
u(z)= & \frac{1}{2 \pi i} \sum_{h=0}^{m-1} \int_{T^{0} \times T^{\prime \prime}} K^{n}\left(t_{0}-z_{0}, z, t_{0}, t^{\prime \prime}\right) u_{h}\left(t_{0}, t^{\prime \prime}\right) d t_{0} d t^{\prime \prime} \\
& +\frac{1}{2 \pi i} \int_{T} K^{m}\left(t_{0}-z_{0}, z, t\right) f(t) d t,
\end{aligned}
$$

where $u_{h}\left(t_{0}, t^{\prime \prime}\right)=\left(\partial / \partial t_{1}\right)^{h} u\left(t_{0}, 0, t^{\prime \prime}\right)$ and the integration paths $T^{0}=T^{0}(a, b), 0<b$ $-a \leqq 2 \pi, T=T^{0} \times T^{\prime}$ and $T^{\prime \prime}$ are defined in $\S 1$. The functions $\left\{K^{h}\left(u^{\prime}, z, t_{0}, t^{\prime \prime}\right)\right.$; $0 \leqq h \leqq m-1\}$ do not depend on $t_{1}$, but for simplicity we denote them by $K^{h}(w, z, t)$ and the same conventions will be used for other functions. We seek for the kernel functions $K^{h}(w, z, t)(0 \leqq h \leqq m)$ in the following form,

$$
K^{h}(w, z, t)=\int_{\Lambda_{0}}^{\infty i \zeta} \exp (-\lambda w) d \lambda \int_{C} \exp \left(-\lambda^{\alpha} \zeta\right) W^{n}(z, t, \lambda, \zeta) d \zeta
$$

where

$$
\alpha=\alpha_{1}=\left(\sigma_{1}-1\right) / \sigma_{1} .
$$

We note that $\alpha$ defined by (6.11) is different from $\alpha$ in $\S 4$. The integration path $C$ in $\zeta$-space in (6.10) will be defined later.

We construct $K^{h}(w, z, t)(0 \leqq h \leqq m)$ by the method described in $\S 3$. As in $\$ 3$, we define the integro-differential operator $\mathcal{L}_{a}\left(z, \lambda, \zeta, \partial_{z}, \partial_{\zeta}, \lambda t_{0}-\lambda \partial_{\lambda}\right)$ for $\alpha=\left(\sigma_{1}-1\right) / \sigma_{1}$. We will determine $W^{h}(z, t, \lambda, \zeta)(0 \leqq h \leqq m)$ by 


$$
\left\{\begin{array}{l}
\mathcal{L}_{\alpha}\left(z, \lambda, \zeta, \partial_{z}, \partial_{\zeta}, \lambda t_{0}-\lambda \partial_{\lambda}\right) W^{h}(z, t, \lambda, \zeta)=\frac{-\delta_{h, m}}{(2 \pi i)^{n+2 \zeta}} \Pi_{i=1}^{n} \frac{1}{\left(t_{i}-z_{i}\right)}, \\
\left.\left(\partial_{z_{1}}\right)^{k} W^{h}(z, t, \lambda, \zeta)\right|_{z_{1}=0}=\frac{-\delta_{h, k}}{(2 \pi i)^{n+1 \zeta}} \Pi_{i=2}^{n} \frac{1}{\left(t_{i}-z_{i}\right)}, \quad(0 \leqq k \leqq m-1) .
\end{array}\right.
$$

Firstly we reduce the initial value problem (6.12) to that with zero initial data. In order to do so we give a lemma.

Lemma 6.1. Let $\varphi(\tau)=\sum_{k=0}^{m} a_{k} \tau^{k}\left(a_{m} \neq 0\right)$. For given $\left\{b_{k} ; 0 \leqq k \leqq m-1\right\}$ there exists uniquely a polynomial $\psi(\tau)$ with degree $<m$ such that

$$
\frac{1}{2 \pi i} \int_{|\tau|=c} \frac{\tau^{k} \varphi(\tau)}{\varphi(\tau)} d \tau=b_{k} \quad \text { for } 0 \leqq k \leqq m-1,
$$

where $c$ is chosen so that all the roots of $\varphi(\tau)=0$ are contained in $\{\tau ;|\tau|<c\}$.

Proof. We may assume $a_{m}=1$. Put

$$
\varphi_{j}(\tau)=\sum_{p=j+1}^{m} a_{p} \tau^{p-j-1} \quad(j=0,1, \cdots, m-1) .
$$

Then we have

$$
\frac{1}{2 \pi i} \int_{1=1=c} \frac{\tau^{k} \varphi_{j}(\tau)}{\varphi(\tau)} d \tau=\delta_{j, k} .
$$

Hence $\psi(\tau)=\sum_{j=0}^{m-1} b_{j} \varphi,(\tau)$ is a desired polynomial. It is easy to show the uniqueness.

We can define by Lemma 6.1 a polynomial $\phi^{n}\left(z^{\prime \prime}, t, \tau\right)$ with degree $<m$ such that

$$
\int_{|\tau|=c} \frac{\tau^{k} \psi^{h}\left(z^{\prime \prime}, t, \tau\right)}{\left(\tau-\tau_{1}\right)^{m}} d \tau=\frac{-\delta_{h, k}}{(2 \pi i)^{n}} \prod_{i=2}^{n} \frac{1}{\left(t_{i}-z_{i}\right)} \quad \text { for } 0 \leqq k \leqq m-1,
$$

where $\tau_{1}$ is a positive constant. Later we will choose $\tau_{1}$ so as to satisfy an inequality and fix it. Using $\phi^{h}\left(z^{\prime \prime}, t, \tau\right)$, define

$$
\tilde{v}^{h}(z, t, \zeta, \tau)=\frac{\phi^{h}\left(z^{\prime \prime}, t, \tau\right)}{\left(\tau-\tau_{1}\right)^{m}} f_{h-1}\left(\zeta+\tau z_{1}\right)
$$

and

$$
\tilde{V}^{n}(z, t, \zeta)=\int_{|\tau|=c} \tilde{v}^{h}(z, t, \zeta, \tau) d \tau, \quad c>\tau_{1} .
$$

Then, if $|\zeta|>\left|c z_{1}\right|$, we have for $0 \leqq k \leqq m-1$

$$
\begin{gathered}
\left.\left(\partial_{1}\right)^{k} \hat{V}^{n}(z, t, \zeta)\right|_{z_{1}=0}=\left.\int_{|\tau|=c} \frac{\tau^{k} \phi^{h}\left(z^{\prime \prime}, t, \tau\right)}{\left(\tau-\tau_{1}\right)^{m}} f_{h-k-1}\left(\zeta+\tau z_{1}\right) d \tau\right|_{z_{1}=0} \\
=\frac{-\delta_{h, k}}{(2 \pi i)^{n}} \Pi_{i=2}^{n} \frac{1}{\left(t_{i}-z_{i}\right)} f_{-1}(\zeta)=\frac{-\delta_{h, k}}{(2 \pi i)^{n+1} \zeta} \Pi_{i=2}^{n} \frac{1}{\left(t_{i}-z_{i}\right)}
\end{gathered}
$$


Hence $V^{h}(z, t, \lambda, \zeta)=W^{h}(z, t, \lambda, \zeta)-\tilde{V}^{h}(z, t, \zeta)$ satisfies the zero initial conditions

$$
\left.\left(\partial_{1}\right)^{k} V^{h}(z, t, \lambda, \zeta)\right|_{z_{1}=0}=0 \quad \text { for } 0 \leqq k \leqq m-1 .
$$

Put

$$
C^{h}(z, t, \lambda, \zeta)=\mathcal{L}_{\alpha}\left(z, \lambda, \zeta, \partial_{z}, \partial_{\zeta}, \lambda t_{0}-\lambda \partial_{\lambda}\right) V^{h}(z, t, \lambda, \zeta) .
$$

It is easy to show that if $|\zeta|>\left|c z_{1}\right|$,

$$
C^{h}(z, t, \lambda, \zeta)=\sum_{k=-1}^{m+h-1} \int_{|\tau|=c} c_{h, k}(z, t, \lambda, \tau) f_{k}\left(\zeta+\tau z_{1}\right) d \tau
$$

where

$$
\left\{\begin{array}{l}
c_{h,-1}(z, t, \lambda, \tau)=\frac{-\delta_{h, m}}{(2 \pi i)^{n+2}}\left(\prod_{i=1}^{n} \frac{1}{\left(t_{i}-z_{i}\right)}\right) \frac{1}{\tau}+\frac{\delta_{h, 0} c_{h,-1}^{*}(z, t, \lambda, \tau)}{\left(\tau-\tau_{1}\right)^{m}}, \\
c_{h, k}(z, t, \lambda, \tau)=\frac{c_{h, k}^{*}(z, t, \lambda, \tau)}{\left(\tau-\tau_{1}\right)^{m}} \text { for } k \neq-1,
\end{array}\right.
$$

where $c_{h, k}^{*}(z, t, \lambda, \tau)(-1 \leqq k \leqq m+h-1)$ are polynomials of $\tau$ with degree $<m$, and $c_{m, k}^{*}(z, t, \lambda, \tau)=0$. Thus the initial value problem (6.12) is equivalent to

$$
\left\{\begin{array}{l}
\mathcal{L}_{\alpha}\left(z, \lambda, \zeta, \partial_{z}, \partial_{\zeta}, \lambda t_{0}-\lambda \partial_{\lambda}\right) W^{h}(z, t, \lambda, \zeta)=C^{h}(t, z, \lambda, \zeta), \\
\left.\left(\partial_{z_{1}}\right)^{k} W^{h}(z, t, \lambda, \zeta)\right|_{z_{1}=0}=0 \quad \text { for } 0 \leqq k \leqq m-1,
\end{array}\right.
$$

where we denote $V^{h}(z, t, \lambda, \zeta)$ again by $W^{h}(z, t, \lambda, \zeta)$. We try to find $W^{h}(z, t, \lambda, \zeta)$ in the form

$$
\left\{\begin{array}{l}
W^{h}(z, t, \lambda, \zeta)=\int_{|\tau|=c} w^{h}(z, t, \lambda, \zeta, \tau) d \tau, \\
w^{h}(z, t, \lambda, \zeta, \tau)=\sum_{n=-1}^{+\infty} w_{n}^{h}(z, t, \lambda, \tau) f_{n}\left(\zeta+\tau z_{1}\right),
\end{array}\right.
$$

where $f_{n}(\zeta)(n \in \boldsymbol{Z})$ are defined by (3.4) and the path $|\tau|=c$ is a circle and encloses $\tau=0$ once, $c$ being large.

Now let us determine $w_{n}^{h}(z, t, \lambda, \tau)(-1 \leqq n<+\infty)$ in (6.25). Substituting $W^{h}(z, t, \lambda, \tau)$ into (6.24), we have as in $\S 3$ (see (3.21) and (3.22))

$$
\begin{aligned}
& G_{0}(z, \lambda, \tau) v_{n}^{h}(z, t, \lambda, \tau) \\
& \quad+\Sigma_{q} G_{q}^{1}\left(z, \partial^{\prime}, \lambda, \tau\right) w_{n-q}^{h}(z, t, \lambda, \tau)=c_{h, n}(z, t, \lambda, \tau),
\end{aligned}
$$

where

$$
\begin{aligned}
& G_{0}(z, \lambda, \tau)=G_{0}^{1}(z, \lambda, \tau) \\
& \quad=\lambda^{m-\left(1-\alpha_{1}\right) d_{m}}\left\{\Sigma_{k, l} \lambda^{\left.-\beta_{k, l}^{1} l\left(-\alpha_{1} z_{1}\right)^{j} \tau^{d_{k, l}} a_{k, l}(z)\right\},}\right.
\end{aligned}
$$




$$
\begin{aligned}
& G_{q}^{1}\left(z, \partial^{\prime}, \lambda, \tau\right)=\lambda^{m-\left(1-\alpha_{1}\right) d m}
\end{aligned}
$$

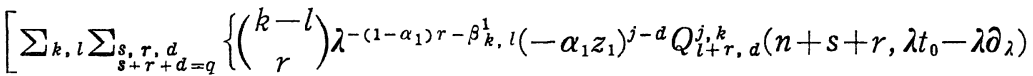

$$
\begin{aligned}
& \left.\left.\tau^{d, l-s-d} a_{k, l, s}\left(z, \partial^{\prime}\right) \partial_{0}{ }^{r}\right\}\right], \quad j=j(k, l) .
\end{aligned}
$$

$G_{0}(z, t, \lambda)$ is a polynomial of $\tau$ with degree $m$ and the coefficient of $\tau^{m}$ is $\lambda^{m-\left(1-\alpha_{1}\right) d_{m}-\beta_{m, m}^{1}} a_{m, m}(z)$ which does not vanish in a neighbourhood of $z=0$ by (6.8). So we conclude that $w_{n}^{h}(z, t, \lambda, \tau)(n \geqq-1)$ are successively determined by (6.26) and each $w_{n}^{h}(z, t, \lambda, \tau)$ is a rational function of $\tau$. By (6.23), if $h=m$, its poles are $\tau=0$ and $\left\{\tau ; G_{0}(z, \lambda, \tau)=0\right\}$ and if $h \neq m$, its poles are $\tau=\tau_{1}$ and $\left\{\tau ; G_{0}(z, \lambda, \tau)=0\right\}$, and the multiplicity of $\tau_{1}$ is $m$.

Now we investigate the roots of the algebraic equation $G_{0}(z, \lambda, \tau)=0$ under the conditions (6.4) and (6.8) in order to analyze the integral representation. Put

$$
\begin{gathered}
F_{0}(z, \lambda, \tau)=\sum_{l=s_{m}}^{m} \lambda^{-\beta_{m, l}^{1}} \tau^{l} a_{m, l}(z), \\
F_{i}(z, \lambda, \tau)=\sum_{\left(k, d_{k, l}\right) \in \Sigma(i)} \lambda^{-\beta_{k, l}^{1} l\left(-\alpha z_{1}\right)^{j} \tau^{d_{k, l}} a_{k, l}(z),} \quad 1 \leqq i \leqq p-1,
\end{gathered}
$$

where $a_{k, l}(z)=a_{k, l}\left(z, \hat{\xi}^{\prime}\right)$ and $j=j(k, l)$, and

$$
F_{i}^{*}(z, \lambda, \tau)=\lambda^{-m+\left(1-\alpha_{1}\right) d} m G_{0}(z, \lambda, \tau)-F_{i}(z, \lambda, \tau) .
$$

We recall that $+\infty=\sigma_{0}>\sigma_{1}>\cdots>\sigma_{p-1}>\sigma_{p}=1, \alpha_{i}=\left(\sigma_{i}-1\right) / \sigma_{i}, m=k_{0}>k_{1}>$ $\cdots>k_{p-1} \geqq 0, \beta_{k_{,} l}^{i}=k_{i-1}-k-\left(d_{k_{i-1}}-d_{k, l}\right)\left(1-\alpha_{i}\right)$ and $\beta_{k_{i}, l_{k_{i}}}^{1}=\beta_{k_{i}}^{1}$ (see $\S 1$ and $\S 3$ ). In the sequel $d_{k_{-1}}=m$ and $\beta_{k_{-1}}^{1}=\left(m-d_{m}\right)\left(1-\alpha_{1}\right)$. We have

Proposition 6.2. There are positive constants $\Lambda_{0}, a_{i}, b_{i},\left(a_{i}>b_{i}, 0 \leqq i \leqq p-1\right)$, $C, r$ and $c$, such that the following holds: Put $U=\left\{z \in C^{n+1} ;|z| \leqq r\right\}, \Upsilon_{i}=$ $\left\{\tau ; b_{i}|\lambda|^{\alpha_{i}-\alpha_{1}}<|\tau|<b_{i-1}|\lambda|^{\alpha_{i-1}-\alpha_{1}}\right\} \quad\left(0 \leqq i \leqq p-1, b_{-1}=+\infty\right), \quad Y_{i}^{\prime}=\left\{\tau ; b_{i}|\lambda|^{a_{i}-\alpha_{1}}<\right.$ $\left.|\tau|<a_{i}|\lambda|{ }^{\alpha_{i}-\alpha_{1}}\right\}$ and $Y_{i}^{\prime \prime}=\left\{\tau ; 3 b_{i}|\lambda|^{\alpha_{i}-\alpha_{1}} / 2<|\tau|<a_{i}|\lambda|^{\alpha_{i}-\alpha_{1}} / 2\right\}$. Let $z \in U$ and $|\lambda| \geqq \Lambda_{0}$. Then it holds that

$$
\left|F_{i}(z, \lambda, \tau)\right| \geqq C|\lambda|^{-\beta_{k i-1}^{1}|\tau|^{d_{k_{i-1}}} \quad(0 \leqq i \leqq p-1)}
$$

on the boundary of $\gamma_{i}$ and

$$
\left|F_{i}^{*}(z, \lambda, \tau)\right| \leqq|\lambda|^{-c}\left|F_{i}(z, \lambda, \tau)\right| \quad(0 \leqq i \leqq p-1)
$$

on the boundary of $Y_{i}^{\prime}$ and all the nonzero roots of $F_{i}(z, \lambda, \tau)=0$ are contained in $Y^{\prime \prime}$.

Proof. Put $\tau=\eta \lambda^{-a_{1}+a_{i}}\left(0 \leqq i \leqq p-1, \alpha_{0}=1\right)$. Then we have by Lemma 3.6 


$$
\begin{aligned}
& F_{i}\left(z, \lambda, \eta \lambda^{-\alpha_{1}+a_{i}}\right) \\
& \quad=\lambda^{-\beta_{k_{\imath}}^{1}-d_{k_{i}}\left(\alpha_{1}-\alpha_{i}\right)}\left\{\sum_{\left((k, l) ;\left(k, d_{k, l}\right) \in \Sigma(i)\right)}\left(-\alpha z_{1}\right)^{j} \eta^{d_{k, l}} a_{k, l}(z)\right\},
\end{aligned}
$$

where $j=j(k, l)$. Since $\beta_{k_{i}}^{1}+d_{k_{i}}\left(\alpha_{1}-\alpha_{i}\right)=\beta_{k_{i-1}}^{1}+d_{k_{i-1}}\left(\alpha_{1}-\alpha_{i}\right)$ by Lemma 3.6 and $d_{k_{i}}=s_{k_{i}}$, that is, $j\left(k_{i}, l_{k_{i}}\right)=0$, there are $a_{i}$ and $b_{i}, a_{i}>b_{i}$. such that $\left|F_{i}(z, \lambda, \tau)\right|$ $\geqq C|\lambda|^{-\beta_{k i-1}^{1}|\tau|^{d_{k i-1}}}$ on $\left\{|\tau|=a_{i}|\lambda|^{-\alpha_{1}+\alpha_{i}}\right\} \cup\left\{|\tau|=b_{i}|\lambda|^{-\alpha_{1}+\alpha_{i}}\right\}$ and all the non zero roots of $F_{i}(z, \lambda, \tau)=0$ are contained in $\left\{\tau ; 3 b_{i}|\lambda|^{\alpha_{i}-a_{1}} / 2<|\tau|<a_{i}|\lambda|^{\alpha_{i}-\alpha_{1}} / 2\right\}$.

Put $\tau=\eta \lambda^{-\alpha_{1}+\alpha_{i-1}}\left(i \geqq 1, \alpha_{0}=1\right)$. Then

$$
\begin{aligned}
& F_{i}\left(z, \lambda, \eta \lambda^{-\alpha_{1}+a_{i-1}}\right)
\end{aligned}
$$

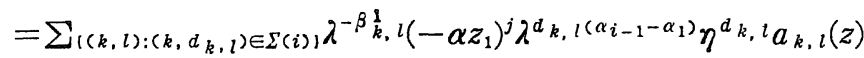

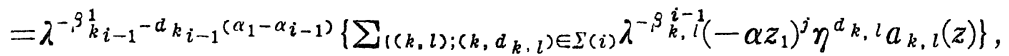

$j=j(k, l)$. Since $\beta_{k, l}^{i-1}>0$ for $(k, l) \neq\left(k_{i-1}, d_{k_{i-1}}\right)$, we have $\left|F_{i}(z, \lambda, \tau)\right| \geqq$ $C|\lambda|^{-\beta_{k}^{1}}{ }_{i-1}|\tau|^{d_{k_{i-1}}}$ on $|\tau|=b_{i-1}|\lambda|^{-\alpha_{1}+\alpha_{i-1}}$ and $|\lambda| \geqq \Lambda_{0}$ for some $\Lambda_{0}$. Thus we have (6.32). It remains to show (6.33). For each term $\lambda^{-\beta k_{k} l}\left(\alpha \tau z_{1}\right)^{j} \tau^{l} a_{k, l}(z)$ in $F_{i}^{*}(z, \lambda, \tau)$, we have $\left|\lambda^{-\beta_{k, l}^{1}}\left(\alpha \tau z_{1}\right)^{j} \tau^{l} a_{k, l}(z)\right| \leqq C|\lambda|^{-\beta_{k, l}^{i}-^{-\beta_{k}}{ }_{i-1}}|\tau|^{d_{k_{i-1}}}$ on the boundary of $\gamma_{\imath}^{\prime}$, where $\beta_{k, l}^{i}>0$. Hence there are a constant $c>0$ and a large $\Lambda_{0}$ such that (6.33) holds for $|\lambda| \geqq \Lambda_{0}$.

Now let $\tau_{i, j}(z) \lambda^{\alpha_{2}-\alpha_{1}}\left(0 \leqq i \leqq p-1,1 \leqq j \leqq d_{k_{i-1}}-d_{k_{i}}\right)$ be non zero roots of $F_{i}(z, \lambda, \tau)=0$. It follows from $d_{k_{p-1}}=0$ that $\sum_{i=0}^{p-1}\left(d_{k_{i-1}}-d_{k_{i}}\right)=m$, and from (6.33) and Rouche's Theorem that there are roots of $G_{0}(z, \lambda, \tau)=0,\left\{\tau_{i, j}(z, \lambda)\right.$; $\left.0 \leqq i \leqq p-1,1 \leqq j \leqq d_{k_{i-1}}-d_{k_{i}}\right\}$, such that $\left\{\tau_{i, j}(z, \lambda) ; 1 \leqq j \leqq d_{k_{i-1}}-d_{k_{i}}\right\} \subset \Upsilon^{\prime \prime}$. More precisely we have

Proposition 6.3. For any small $\eta>0$ there are $\Lambda_{0}$ and $r$ such that $\left|\tau_{i, j}(z, \lambda) \lambda^{\alpha_{1}-a_{i}}-\tilde{\tau}_{i, j}(0)\right|<\eta / 2$ for $z \in U=\{|z| \leqq r\}$ and $|\lambda| \geqq \Lambda_{0}$.

Choose $\tau_{1}$ in (6.16) so that $b_{1}<\tau_{1}<a_{1}$ and fix it.

Define for $0 \leqq \imath \leqq p-1$

$$
\begin{gathered}
K_{i}(\eta)=\left\{\tau ;\left|\tau-\tau_{i, j}(0)\right|<\eta \text { for } 1 \leqq j \leqq d_{k_{i-1}}-d_{k_{i}}\right\}, \\
K_{1}^{*}(\eta)=K_{1}(\eta) \cup\left\{\left|\tau-\tau_{1}\right|<\eta\right\} \text { and } K_{i}^{*}(\eta)=K_{i}(\eta) \quad \text { for } i \neq 1
\end{gathered}
$$

and sets $\tau(i)(0 \leqq i \leqq p)$, by using the constants $a_{i}$ and $b_{i}$ in Proposition 6.2 and small $\eta>0$ : for $0 \leqq i \leqq p-1$

$$
\begin{aligned}
& \tau(i)=\left\{\tau ; b_{i}|\lambda|^{\alpha_{i}-a_{1}} \leqq|\tau|<b_{i-1}|\lambda|^{\alpha_{i-1}-\alpha_{1}}\right\} \\
& \cap\left\{\tau ;\left|\tau-\stackrel{\tau}{\tau}, j_{i, j}(0) \lambda^{\alpha_{i}-\alpha_{1}}\right|>\eta|\lambda|^{a_{i}-a_{1}} \text { for } 1 \leqq j \leqq d_{k_{i-1}}-d_{k_{i}}\right\}, \\
& \tau(p)=\left\{\tau ;|\tau|<b_{p-1}|\lambda|^{\alpha p-1-a_{1}}\right\} .
\end{aligned}
$$

Proposition 6.3 means that for given small $\eta$ 


$$
\begin{aligned}
& \left\{\tau_{i, j}(z, \lambda) ; 1 \leqq j \leqq d_{k_{i-1}}-d_{k_{i}}\right\} \subset \lambda^{a_{2}-a_{1}} K_{i}(\eta / 2) \\
& \subset Y_{i}^{\prime \prime}=\left\{\tau ; 3 b_{i}|\lambda|^{a_{i}-\alpha_{1}} / 2<|\tau|<a_{i}|\lambda|^{a_{i}-\alpha_{1}} / 2\right\}
\end{aligned}
$$

for $z \in U=\{|z| \leqq r\}$ and $|\lambda| \geqq \Lambda_{0}$. We also define the sets $\Xi(i)$ and $\Xi$

$$
\left\{\begin{array}{l}
\Xi(i)=\left\{(z, t, \lambda, \tau) ;(z, t) \in X,|\lambda| \geqq \Lambda_{0}, \tau \in \tau(i)\right\}, \quad 0 \leqq i \leqq p, \\
\Xi=\bigcup_{i=0}^{p-1} \Xi(i),
\end{array}\right.
$$

where $X=\left\{(z, t) \in \boldsymbol{C}^{n+1} \times \boldsymbol{C}^{n-1} ;|z| \leqq r_{1},\left|t_{0}\right| \leqq r_{3}, r_{2} \leqq\left|t_{i}\right| \leqq r_{3}(1 \leqq i \leqq n)\right\}$ and $r$ is small and $\Lambda_{0}$ is large, if necessary.

We have by the same method as in Proposition 6.2 .

Proposition 6.4. On $\left\{(z, \lambda, \tau) ;|z| \leqq r,|\lambda| \geqq \Lambda_{0}, \tau \in \tau(i)\right\}$,

$$
\left|G_{0}(z, \lambda, \tau)\right| \geqq C|\lambda|^{m-\left(1-a_{1}\right) d_{m}-\beta_{k_{i-1}}^{1}|\tau|^{d_{k}}{ }_{i-1}}
$$

Let us define sectors $S_{i}(0 \leqq i \leqq p-1)$, which are used in the later part of this sections for analysis of the integral representation. We defined sectors $S_{i}$ $(1 \leqq i \leqq p-1)$ in $[12]$, where $S_{0}$ was not defined, but the following arguments are the same as in [12]. To define $S_{\imath}$ we give two lemmas.

Lemma 6.5. There is an $\omega_{0}\left(\left|\omega_{0}\right|=1\right)$ such that $\arg \left(\tau_{1} \omega_{0}\right) \neq \pi-\pi \alpha_{1}(\bmod 2 \pi)$ and $\arg \left(\stackrel{\circ}{i, j}_{i}(0) \omega_{0}\right) \neq \pi-\pi \alpha_{1}(\bmod 2 \pi)$ for all $0 \leqq i \leqq p-1$ and $1 \leqq j \leqq d_{k_{i-1}}-d_{k_{i}}$.

Proof. Put $B=\left\{\tau_{i, j}(0) ; 0 \leqq i \leqq p-1,1 \leqq j \leqq d_{k_{2-1}}-d_{k_{i}}\right\} \cup\left\{\tau_{1}\right\}, L_{i}=\left\{r e^{2\left(\pi-\pi a_{2}\right)}\right.$; $r \geqq 0\}$ and $L=\cup_{i=0}^{p-1} L_{i} . \quad B$ is a finite set of nonzero points and $L$ is a finite set of half lines. So we can find an $\omega_{0}\left(\left|\omega_{0}\right|=1\right)$ such that $\omega_{0} B \cap L=\varnothing$. This implies the assertion.

We have from Lemma 6.5

Lemma 6.6. There are $\omega_{1}\left(\left|\omega_{1}\right|=1\right)$ and positive numbers $r, \varepsilon_{1}$ and $\Lambda_{0}$ such that $\arg \tau_{1} \omega \neq \pi-\pi \alpha_{1}(\bmod 2 \pi)$ and $\arg \tau_{i, j}(z, \lambda) \omega \neq \pi-\pi \alpha_{i}(\bmod 2 \pi)$ for all $|\lambda|$ $\geqq \Lambda_{0},|z| \leqq r$ and $\left|\omega-\omega_{1}\right|<\varepsilon_{1}$.

Thus we have

Proposition 6.7. There are $\eta, \varepsilon_{1}>0, \hat{z}_{1} \neq 0$ and open sectors $S_{i}(0 \leqq i \leqq p-1)$ with the vertex 0 in $C^{1}$ such that $S_{i} \ni e_{i}=e^{i\left(\pi-\pi a_{2}\right)}$ and $\bar{S}_{i} \cap\left(-z_{1} K_{i}^{*}(\eta / 2)\right)=\varnothing$ $(0 \leqq i \leqq p-1)$ for $\left|z_{1}-\hat{z}_{1}\right|<\varepsilon_{1}$.

Now we retun to construction of $w^{h}(z, t, \lambda, \tau)$ in (6.25). As for estimates of $w_{n}^{h}(z, t, \lambda, \tau)$ we have

Proposition 6.8. There exist positive constants $A, B$ and $c$ such that the 
following estimates of $w_{n}^{h}(z, t, \lambda, \tau)$ hold in $\Xi$. For $0 \leqq h \leqq m-1$

$$
\left|\lambda^{c}\left(\tau-\tau_{1}\right)^{m} w_{n}^{h}(z, t, \lambda, \tau)\right| \leqq A B^{n+1} n !\left(\sum_{r=0}^{n+1}\left|\lambda t_{0}\right|^{r} / r !\right) /|\tau|^{n+1}
$$

and

$$
\left|\lambda^{c} \tau w_{n}^{m}(z, t, \lambda, \tau)\right| \leqq A B^{n+1} n !\left(\sum_{r=0}^{n+1}\left|\lambda t_{0}\right|^{r} / r !\right) /|\tau|^{n+1}
$$

We refer the proof of Proposition 6.8 to $\S 7$. The convergence of $w^{h}(z, t, \lambda, \zeta, \tau)=\sum_{n=-1}^{\infty} w_{n}^{h}(z, t, \lambda, \tau) f_{n}\left(\zeta+\tau z_{1}\right)$ follows from Proposition 6.8.

Proposition 6.9. (1) There is a constant $c^{*}>0$ such that $w^{h}(z, t, \lambda, \zeta, \tau)$ converges in $\left\{(z, t, \lambda, \zeta, \tau) ;(z, t, \lambda, \tau) \in \Xi, \tau \neq 0, \tau_{1}\right.$ and $\left.0<\left|\zeta+\tau z_{1}\right|<c^{*}|\tau|\right\}$.

(2) There exist positive constants $A, B, C_{1}$ and $c^{\prime}$ such that the following estimates holds:

$$
\begin{aligned}
& \left|\left(\tau-\tau_{1}\right)^{m}\left\{w^{h}(z, t, \lambda, \zeta, \tau)-w_{-1}^{h}(z, t, \lambda, \tau) f_{-1}\left(\zeta+\tau z_{1}\right)\right\}\right| \\
& \leqq \frac{A}{|\tau|}|\lambda|^{c^{\prime}} \exp \left(c^{*} B\left|\lambda t_{0}\right|\right)\left(\left|\log \left(\zeta+\tau z_{1}\right)\right|+C_{1}\right) \quad \text { for } 0 \leqq h \leqq m-1
\end{aligned}
$$

and

$$
\begin{aligned}
& \left|\tau\left\{w^{m}(z, t, \lambda, \zeta, \tau)-w_{-1}^{m}(z, t, \lambda, \tau) f_{-1}\left(\zeta+\tau z_{1}\right)\right\}\right| \\
& \quad \leqq \frac{A}{|\tau|}|\lambda|^{c^{\prime}} \exp \left(c^{*} B\left|\lambda t_{0}\right|\right)\left(\left|\log \left(\zeta+\tau z_{1}\right)\right|+C_{1}\right) .
\end{aligned}
$$

Proof. The following argument is the same as in Proposition 4.2. We have, by Proposition 6.8,

$$
\begin{aligned}
& \sum_{n=0}^{\infty}\left|\lambda^{c}\left(\tau-\tau_{1}\right)^{m} w_{n}^{h}(z, t, \lambda, \tau)\left(\zeta+\tau z_{1}\right)^{n} / n !\right| \\
& \quad \leqq \frac{A}{|\tau|} \sum_{n=0}^{\infty}\left|B\left(\zeta+\tau z_{1}\right) / \tau\right|^{n}\left(\sum_{r=0}^{n+1}\left|\lambda t_{0}\right|^{r} / r !\right) .
\end{aligned}
$$

If $\left|\left(\zeta+\tau z_{1}\right) / \tau\right|<c^{*}$ and $c^{*} B<1 / 2$, the above series converges and we have (6.43). We have (6.44) by the same method.

Remark 6.10. Since $w_{n}^{h}(z, t, \lambda, \tau)(h \neq m, n \geqq-1)$ are holomorphic at $\tau=0$, it follows from the maximal principle of holomorphic functions and (6.41) that $w^{h}(z, t, \lambda, \zeta, \tau)(h \neq m)$ are holomorphic at $\tau=0$. Since $w_{n}^{m}(z, t, \lambda, \tau)(n \geqq-1)$ have a single pole at $\tau=0, w^{m}(z, t, \lambda, \zeta, \tau)$ has a single pole at $\tau=0$ by (6.42).

We have obtained $w^{h}(z, t, \lambda, \zeta, \tau)$ in (6.25). So we proceed to construction of $W^{h}(z, t, \lambda, \zeta)$. Define

$$
W_{i}^{h}(z, t, \lambda, \zeta)=\int_{|\tau|=c_{i}} w^{h}(z, t, \lambda, \zeta, \tau) d \tau \quad(0 \leqq i \leqq p),
$$

where $a_{i}|\lambda|^{\alpha_{i}-\alpha_{1}}<c_{\imath}<b_{i-1} \mid \lambda_{i}^{a_{i-1}-\alpha_{1}}$ and constants $a_{i}$ and $b_{i}$ are those in 
Proposition 6.2, $a_{p}=0, b_{-1}=+\infty$. Put

$$
W^{h}(z, t, \lambda, \zeta)=W_{0}^{h}(z, t, \lambda, \zeta) .
$$

Now we study the domain to which $W^{h}(z, t, \lambda, \zeta)(0 \leqq h \leqq m)$ are holomorphically extensible as functions of $\zeta$. So we often omit the other variables. We always assume $(z, t, \lambda) \in X_{\Lambda_{0}}, \quad X_{\Lambda_{0}}=X \times \tilde{\Lambda}_{0}^{*}, \quad \Lambda_{0}^{*}=\left\{\lambda \in C^{1} ;|\lambda| \geqq \Lambda_{0}\right\}, \quad X=$ $\left\{(z, t) ;|z| \leqq r_{1}, \quad t_{0}\left|\leqq r_{3}, r_{2} \leqq\right| t_{i} \mid \leqq r_{3}\right\}$, and put

$$
Z(i)=\left\{\zeta ; a_{i}\left|z_{1}\right||\lambda|^{a_{i-a_{1}}}<|\zeta|<\left(C-\left|z_{1}\right|\right) b_{i-1}|\lambda|^{a_{i-1}-a_{1}}\right\}, \quad 0 \leqq i \leqq p,
$$

for a $C>0$, which is chosen suitably. Firstly we have from Proposition 6.9 and Remark 6.10.

Proposition 6.11. (1) $W_{i}^{h}(\zeta)(i \neq p)$ is holomorphic in $\tilde{Z}(i)$.

(2) $W_{p}^{h}(\zeta) \equiv 0(h \neq m)$ and $W_{p}^{m}(\zeta)=a(\zeta) / \zeta+b(\zeta) \log \zeta$ in $\tilde{Z}(p)$, where $a(\zeta)$ and $b(\zeta)$ are holomorphic.

Proof. Suppose that $a_{i}|\lambda|^{\alpha_{2}-\alpha_{1}}<|\tau|<b_{i-1}|\lambda|^{a_{2-1}-a_{1}},|\zeta|>\left|\tau z_{1}\right|$ and $|\zeta|+$ $\left|\tau z_{1}\right|<c^{*}|\tau|$, $c^{*}$ being that in Proposition 6.9. Then $w^{h}(z, t, \lambda, \zeta, \tau)$ is holomorphic. Integrating it on $|\tau|=r, a_{i}|\lambda|^{a_{i-a_{1}}}<r<b_{\imath-1}|\lambda|^{a_{2-1}-a_{1}}$, we have $W_{l}^{h}(\zeta)$ $\in \mathcal{O}\left(\tilde{Z}^{r}\right), Z^{r}=\left\{\zeta ; r\left|z_{1}\right|<|\zeta|<\left(c^{*}-\left|z_{1}\right|\right) r\right\}$. So varying $r$, we have (1) for a constant $C>0$. Since $w^{h}(z, t, \lambda, \zeta, \tau)(h \neq m)$ is holomorphic on $\tau(p), W_{p}^{h}(\zeta) \equiv 0$. $w^{m}(z, t, \lambda, \zeta, \tau)$ has a single pole at $\tau=0$ in $\tau(p)$, that is, $w^{m}(z, t, \lambda, \zeta, \tau)=$ $(1 / \tau) \sum_{n=-1}^{+\infty} w_{n}^{m}(z, t, \lambda, \tau) f_{n}\left(\zeta+\tau z_{1}\right)$, where $\tilde{w}_{n}^{m}(z, t, \lambda, \tau)(n \geqq-1)$ are holomorphic in $\tau(p)$ (see Remark 6.10). Hence

$$
W_{p}^{m}(z, t, \lambda, \zeta)=\int_{1=1=c_{p}} u^{m}(z, t, \lambda, \zeta, \tau) d \tau=\sum_{n=-1}^{+\infty} \tilde{w}_{n}^{m}(z, t, \lambda, 0) f_{n}(\zeta)
$$

which implies (2).

Put

$$
W_{i, i+1}^{h}(\zeta)=W_{i}^{h}(\zeta)-W_{i+1}^{h}(\zeta), \quad i=0,1, \cdots, p-1 .
$$

It follows from Proposition 6.11 that $W_{i, i+1}^{h}(\zeta)$ is holomorphic in $\tilde{Z}_{i}^{\prime}, Z_{i}^{\prime}=$ $\left\{\zeta ; a_{i}\left|z_{1}\right||\lambda|^{a_{2}-a_{1}}<|\zeta|<\left(C-\left|z_{1}\right|\right) b_{i}|\lambda|^{\alpha_{i}-\alpha_{1}}\right\}$, for small $z_{1}$. We have by the deformation of the integration path to $\partial\left(K_{i}^{*}(\eta) \lambda^{\alpha_{i}-a_{1}}\right)$ (see (6.35)).

$$
W_{i, i+1}^{h}(\zeta)=\int_{\partial\left(K_{i}^{*}(\eta) \lambda^{a_{i}-\alpha_{1}},\right.} w^{h}(z, t, \lambda, \zeta, \tau) d \tau .
$$

From (6.50) we have

Proposition 6.12. $W_{i, i+1}^{h}(\zeta)$ is holomorphic on $\tilde{Z}_{i, i+1}$ for small $z_{1}, Z_{i, i+1}=$ $\left\{\zeta \notin-z_{1} K_{i}^{*}(\eta) \lambda^{a_{i}-a_{1}} ;|\zeta|<\left(C-\left|z_{1}\right|\right) b_{i}|\lambda|^{\alpha_{i}-\alpha_{1}}\right\}$.

Considering Propositions 6.11 and 6.12 and (6.49), we have 
Proposition 6.13. $W_{i}^{h}(\zeta)(i \neq p)$ has a holomorphic prolongation to $\tilde{Z}_{i}$,

$$
\begin{aligned}
Z_{i}= & \left\{\zeta \notin-z_{1} K_{i}^{*}(\eta) \lambda^{a_{i}-\alpha_{1}} ;\right. \\
& \left.a_{i+1}\left|z_{1}\right||\lambda|^{\alpha_{i+1}-\alpha_{1}}<|\zeta|<\left(C-\left|z_{1}\right|\right) b_{i-1}|\lambda|^{\alpha_{i-1}-a_{1}}\right\},
\end{aligned}
$$

for small $z_{1}$. Moreover $W_{p-1}^{h}(\zeta)(0 \leqq h \leqq m-1)$ are holomorphic at $\zeta=0$.

Hence, by using the relation

(6.52) $W^{h}(\zeta)=W_{0}^{h}(\zeta) \quad$ in $\tilde{Z}(0)$

$$
\begin{aligned}
& =W_{0,1}^{h}(\zeta)+W_{1}^{h}(\zeta) \quad \text { in } \widetilde{Z_{0,1} \cap Z(1)} \\
& =W_{0,1}^{h}(\zeta)+W_{1,2}^{h}(\zeta)+W_{2}^{h}(\zeta) \quad \text { in } \widetilde{Z_{1,2} \cap Z(2)} \\
& =\cdots \cdots \\
& =W_{0,1}^{h}(\zeta)+W_{1,2}^{h}(\zeta)+W_{2,3}^{h}(\zeta)+\cdots+W_{p-2, p-1}^{h}(\zeta)+W_{p-1, p}^{h}(\zeta)+W_{p}^{h}(\zeta) \\
& \quad \text { in } \tilde{Z}_{p-1, p},
\end{aligned}
$$

we can prolonge $W^{h}(\zeta)(0 \leqq h \leqq m)$ holomorphically. Thus we have

Proposition 6.14. There is a convering $\hat{Z}$ of $Z=\cup_{i=0}^{p} Z_{i}$ such that each $W^{h}(\zeta)(0 \leqq h \leqq m)$ has a holomorphic prolongation to $\hat{Z}$ as a function of $\zeta, W^{h}(\zeta)$ $(h \neq m)$ is holomorphic at $\zeta=0$ in $\hat{Z}$ and the singularity of $W^{m}(\zeta)$ at $\zeta=0$ is polar and logarithmic, that is, $W^{m}(\zeta)=a(\zeta) / \zeta+b(\zeta) \log \zeta$ at $\zeta=0$.

Define

$$
\hat{K}_{\theta}^{h}(z, t, \lambda)=\int_{C_{0}(\theta)} \exp \left(-\lambda^{\alpha} \zeta\right) W^{h}(z, t, \lambda, \zeta) d \zeta,
$$

where $C_{0}(\theta)=\left\{\zeta=d_{-1} e^{i(\theta+2 \pi s)} \lambda^{1-\alpha_{1}} ; 0 \leqq s \leqq 1\right\}, a_{0}\left|z_{1}\right| \leqq d_{-1}$. We have

Proposition 6.15. The following estimates hold:

$$
\left|\hat{K}_{\theta}^{h}(z, t, \lambda)\right| \leqq A(1+|\lambda|)^{N} \exp \left(d_{-1}|\lambda|+c^{*} B\left|\lambda t_{0}\right|\right) \quad \text { for }|\lambda| \geqq \Lambda_{0},
$$

and if $|\arg \lambda+\theta|<\pi / 2$,

$$
\left|\hat{K}_{\theta}^{h}(z, t, \lambda)\right| \leqq A(1+|\lambda|)^{N} \exp \left(a_{0}\left|\lambda z_{1}\right|+c^{*} B\left|\lambda t_{0}\right|\right),
$$

where $c^{*}$ and $B$ are those in Proposition 6.9.

Proof. The estimate (6.54) follows from Proposition 6.9. We can deform the path $C_{0}(\theta)$ to $C^{\prime}(\theta)$, starting at $\zeta=d_{-1} e^{i \theta} \lambda^{1-\alpha_{1}}$, going to $a_{0}\left|z_{1}\right| e^{i \theta} \lambda^{1-a_{1}}$, enclosing the origin $\zeta=0$ once on $|\zeta|=a_{0}\left|z_{1} \lambda^{1-\alpha_{1}}\right|$ and going from $a_{0}\left|z_{1}\right| e^{i(\theta+2 \pi)} \lambda^{1-\alpha_{1}}$ to $d_{-1} e^{i(\theta+2 \pi)} \lambda^{1-a_{1}}$. We have (6.55) by this deformation. 
Now we can define $K^{h}(w, z, t), 0 \leqq h \leqq m$, (see (6.9), (6.10) and (6.53))

$$
K_{\theta}^{h}(w, z, t)=\int_{\Lambda_{0}}^{\infty i \dot{\varphi}} \exp (-\lambda w) \hat{K}_{\theta}^{h}(z, t, \lambda) d \lambda, \quad|\phi+\theta|<\frac{\pi}{2},
$$

which depends on $\theta$. We have from Proposition 6.15,

Proposition 6.16. $K_{\theta}^{h}(w, z, t)$ is holomorphic in $\{(w, z, t)$; $|\arg w-\theta|<\pi$, $\left.|w|>a_{0}\left|z_{1}\right|+c^{*} B\left|t_{0}\right|\right\}$.

Proposition 6.17. If $c^{*}>0$ in Proposition 6.15 is small, then the following identities hold:

$$
\begin{gathered}
L\left(z, \partial_{z}\right) K_{\theta}^{h}\left(t_{0}-z_{0}, z, t\right) \equiv \frac{\delta_{h, m}}{(2 \pi i)^{n+1}} \frac{-1}{t_{0}-z_{0}} \prod_{i=1}^{n} \frac{1}{t_{i}-z_{i}}, \\
\left.\left(\partial_{1}\right)^{k} K_{\theta}^{h}\left(t_{0}-z_{0}, z, t\right)\right|_{z_{1}=0} \equiv \frac{\delta_{h, k}}{(2 \pi i)^{n}} \frac{-1}{t_{0}-z_{0}} \Pi_{i=2}^{n} \frac{1}{t_{i}-z_{i}} \text { for } 0 \leqq k \leqq m-1,
\end{gathered}
$$

where $\equiv$ means modulo holomorphic functions on $X$.

Proof. By repeating the same method as in the proof of Proposition 4.5, we have

$$
\begin{aligned}
& L\left(z, \partial_{z}\right) K_{\theta}^{h}\left(t_{0}-z_{0}, z, t\right) \\
& =\int_{\Lambda_{0}}^{\infty i \phi} \exp \left(-\lambda\left(t_{0}-z_{0}\right)\right) d \lambda \int_{C_{0}(\theta)} \exp \left(-\lambda^{a} \zeta\right) \frac{-\delta_{h, m}}{(2 \pi i)^{n+2 \zeta}} \prod_{i=1}^{n} \frac{1}{t_{i}-z_{i}} d \zeta \\
& \quad+\int_{\Lambda_{0}}^{\infty e^{i \psi}} \exp \left(-\lambda\left(t_{0}-z_{0}\right)-\lambda d_{-1} e^{i \theta}\right) \tilde{K}^{h}(z, t, \lambda) d \lambda,
\end{aligned}
$$

where $\left|\widetilde{K}^{h}(z, t, \lambda)\right| \leqq C(1+|\lambda|)^{N} \exp \left(c^{*} B\left|\lambda t_{0}\right|\right)$ for some $N$. If $c^{*}>0$ is small such as $d_{-1}>c^{*} B\left|t_{0}\right|$, then, by putting $\phi=-\theta$ in (6.56),

$$
\int_{\Lambda_{0}}^{\infty e^{-i \theta}} \exp \left(-\lambda w-\lambda d_{-1} e^{i \theta}\right) \tilde{K}^{h}(z, t, \lambda) d \lambda
$$

is holomorphic at $w=0$. It is easy to get (6.58).

Define, putting $\theta=-\pi$,

$$
K^{h}(w, z, t)=K_{-\pi}^{h}(w, z, t),
$$

and $\delta_{0}=\sin ^{-1}\left(c^{*} B\right)$, where $c^{*}>0$ is small. Now let $u(z) \in \mathcal{O}\left(\Omega\left(\theta_{0}\right)\right)$ be a solution of (6.1) with $f(z) \in \mathcal{O}\left(\Omega\left(\theta_{0}\right)\right), \theta_{0}>\delta_{0}$. Define

$$
\begin{aligned}
u_{-\pi}(z)= & \sum_{n=0}^{m} u_{-\pi}^{h}=\sum_{h=0}^{m-1} \int_{T_{0} \times T^{\prime \prime}} K^{h}\left(t_{0}-z_{0}, z, t\right) u^{h}\left(t_{0}, 0, t^{\prime \prime}\right) d t_{0} d t^{\prime \prime} \\
& +\int_{T_{0} \times T^{\prime}} K^{m}\left(t_{0}-z_{0}, z, t\right) f\left(t_{0}, t^{\prime}\right) d t_{0} d t^{\prime}
\end{aligned}
$$


where $T_{0}=T_{0}(a, b)$ and $u^{h}\left(t_{0}, 0, t^{\prime \prime}\right)=\partial_{1}^{h} u\left(t_{0}, 0, t^{\prime \prime}\right)$. The formula (6.60) is an integral representation of $u(z)$ in (6.1). We have from Proposition 6.17.

Theorem 6.18. $u_{-\pi}(z)$ defined by (6.60) satisfies

$$
\begin{gathered}
L\left(z, \partial_{z}\right) u_{-\pi}(z) \equiv f(z), \\
\left.\left(\partial_{1}\right)^{k} u_{-\pi}(z)\right|_{z_{1}=0} \equiv \partial_{1}^{k} u\left(z_{0}, 0, z^{\prime \prime}\right) \quad \text { for } 0 \leqq k \leqq m-1,
\end{gathered}
$$

where $\equiv$ means modulo holomorphic functions at $z=0$, and $u(z)-u_{-\pi}(z)=v(z) \in$ $\mathcal{O}(U)$ in a neighbourhood of the origin.

Proof. We have by the method used to show Proposition 4.5

$$
\begin{aligned}
L\left(z, \partial_{z}\right) u_{-\pi}(z) & =\frac{-1}{(2 \pi i)^{n+1}} \int_{T_{0} \times T^{\prime}} \frac{f(t)}{t_{0}-z_{0}} \Pi_{i=1}^{n} \frac{1}{t_{i}-z_{i}} d t_{0} d t^{\prime}, \\
& =f(z)+\frac{1}{(2 \pi i)^{n+1}} \int_{T_{0}^{c} \times T^{\prime}} \frac{f(t)}{t_{0}-z_{0}} \Pi_{i=1}^{n} \frac{1}{t_{\imath}-z_{i}} d t_{0} d t^{\prime} .
\end{aligned}
$$

This means (6.61). We have (6.62) from (6.58). It follows from the uniqueness of Cauchy problem that $u(z)-u_{-\pi}(z)=v(z) \in \mathcal{O}(U)$ in a neighbourhood of the origin.

Remark 6.19. We can show that if $\left|\theta-\theta^{\prime}\right|$ is small then $K_{\theta}^{h}(w, z, t)-$ $K_{\theta^{\prime}}^{h}(w, z, t)$ is holomorphic in a neighbourhood of $w=0$. So the representation (6.60) is holomorphically extensible to wider domains, which will be done by replacing $K_{-\pi}(w, z, t)$ by $K_{\theta}(w, z, t)$.

We investigate $\hat{K}^{h}(z, t, \lambda)=\hat{K}_{-\pi}^{h}(z, t, \lambda)$ more precisely, by using Proposition 6.7. So in the sequel we restrict $(z, t, \lambda)$ to the set

$$
\tilde{X}_{\Lambda_{0}}^{\prime}=X^{\prime} \times \tilde{\Lambda}_{0}^{*}, \quad X^{\prime}=\left\{(z, t, \lambda) ;(z, t) \in X,\left|z_{1}-\hat{z}_{1}\right|<\varepsilon_{1}\right\} .
$$

The following arguments are similar to that in [12]. Firstly we decompose integration path $C_{0}=C_{0}(-\pi)$ in (6.53), and secondly according to the decomposition of $C_{0}$, decompose $\hat{K}^{h}(z, t, \lambda), \hat{K}^{h}(z, t, \lambda)=\sum_{i, s} \hat{K}_{i, s}^{h}(z, t, \lambda)$. We investigate each $\hat{K}_{i, s}^{h}(z, t, \lambda)$. So we define some paths in $\zeta$-space. For a path $C=\{\zeta(s) ; 0 \leqq s \leqq 1\}$ and $a \in C, a C=\{a \zeta(s) 0 \leqq s \leqq 1\}$. Put $A_{i}=\{\zeta(s)=$

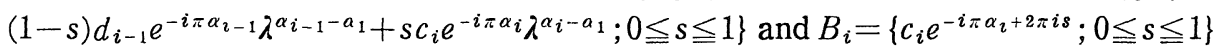
$(0 \leqq i \leqq p-1)$, where $c_{i}>a_{i}\left|z_{1}\right|>b_{2}\left|z_{1}\right|>d_{i}, d_{-1}>a_{0}\left|z_{1}\right|$ and $\alpha_{-1}=\alpha_{0}=1$. Put $C_{i}=A_{i}+\lambda^{a_{2}-\alpha_{1}} B_{2}-e^{2 \pi i} A_{i}(1 \leqq i \leqq p-1)$ and $C_{p}=\left\{\zeta(s)=d_{p-1} \lambda^{\alpha_{p-1}-\alpha_{1}} e^{-i \pi a_{p-1}+2 \pi i s}\right.$; $0 \leqq s \leqq 1\}$ (see Fig. 6.1). The path $C_{0}$ and $C_{p}$ were not used in [12]. 


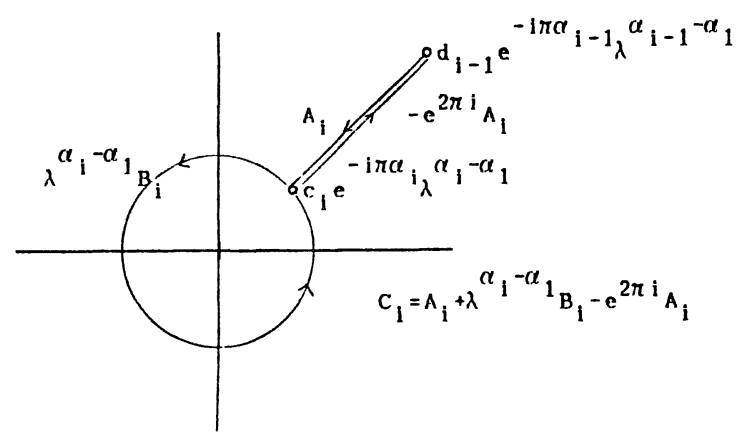

Fig. 6.1.

We note that the singularity of $W^{h}(z, t, \lambda, \zeta)$ with respect to $\zeta$ are in $\left(\cup_{i=0}^{p-1}-z_{1} \lambda^{a_{2}-\alpha_{1}} K_{i}^{*}(\eta / 2)\right) \cup\{\zeta=0\}$. Define

$$
\begin{aligned}
\hat{K}_{i}^{h}(z, t, \lambda) & =\int_{C_{i}} \exp \left(-\lambda^{\alpha_{1} \zeta}\right) W^{h}(z, t, \lambda, \zeta) d \zeta \\
& =\left(\int_{A_{i}}+\int_{\lambda^{\alpha}{ }^{\alpha_{i}-\alpha_{1_{B}}}}+\int_{-e^{2 \pi i_{A_{i}}}}\right) \exp \left(-\lambda^{a_{1} \zeta}\right) W^{h}(z, t, \lambda, \zeta) d \zeta .
\end{aligned}
$$

Let us deform the path $\lambda^{\alpha_{i}-\alpha_{1}} B_{i}$ to another. We give

Lemma 6.20. The path $\lambda^{a_{i-\alpha} \alpha_{1}} B_{i}$ can be deformed homotopically to $B_{i}^{*}$ such that: $B_{i}^{*}=\lambda^{\alpha_{i}-\alpha_{1}} B_{i}^{\prime}+C_{i+1}+\lambda^{a_{i}-\alpha_{1}} B_{i}^{\prime \prime}$, where $B_{i}^{\prime}$ and $B_{i}^{\prime \prime}$ are independent of $\lambda$, contained in $\left\{\zeta ; d_{i} \leqq|\zeta| \leqq c_{i}\right\}$ and $\left(B_{i}^{\prime} \cup B_{i}^{\prime \prime}\right) \cap \bar{S}_{i}=\varnothing$, and

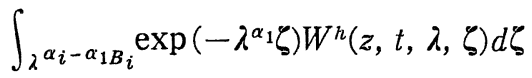

$$
\begin{aligned}
& =\left(\int_{\lambda \alpha_{i}-\alpha_{B_{B}^{\prime}}}+\int_{C_{i+1}}+\int_{\lambda^{\alpha_{i}-\alpha_{1 B_{i}^{\prime \prime}}}}\right) \exp \left(-\lambda^{\alpha_{1} \zeta}\right) W^{h}(z, t, \lambda, \zeta) d \zeta .
\end{aligned}
$$

Proof. As we remarked, the singularities inside of $C_{i}$ of $W^{h}(z, t, \lambda, \zeta)$ are in $\left(\cup_{q=i}^{p-1}-z_{1} \lambda^{\alpha} q^{-\alpha_{1}} K_{q}^{*}(\eta / 2)\right) \cup\{\zeta=0\}$ and the set $\left(\bigcup_{q=i+1}^{p-1}-z_{1} \lambda^{\alpha_{q}-\alpha_{1}} K_{q}^{*}(\eta / 2)\right) \cup\{\zeta=0\}$ are inside of $C_{i+1}$. So we can deform $B_{i}$ so that $B_{i}^{\prime}$ and $B_{i}^{\prime \prime}$ encloses $-z_{1} K_{i}^{*}(\eta / 2)$. From Proposition 6.7, we can take $B_{i}^{\prime}$ and $B_{i}^{\prime \prime}$ so that $\left(B_{i}^{\prime} \cup B_{i}^{\prime \prime}\right) \cap \bar{S}_{i}=\varnothing$ (see Fig 6.2). 


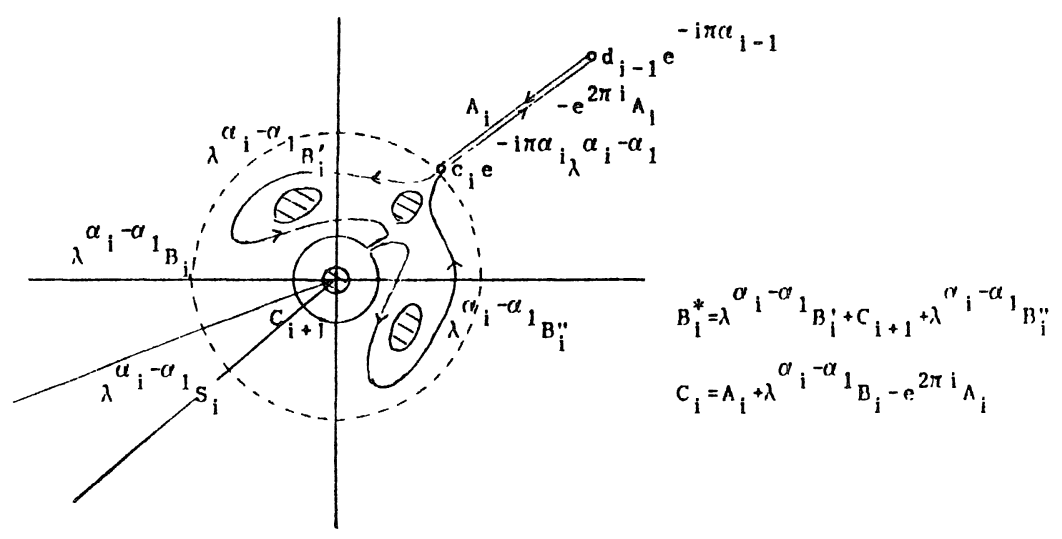

Fig. 6.2.

The singularities of $W^{h}(z, t, \lambda, \zeta)$ inside of $C_{i}$ are in the parts of oblique lines in Fig. 6.2.

Thus we have

Proposition 6.21. $\hat{K}^{n}(z, t, \lambda)$ is represented in the following form:

$$
\begin{aligned}
\hat{K}^{h}(z, t, \lambda)= & \sum_{i=0}^{p-1}\left\{\left(\int_{A_{i}}+\int_{-e^{2 \pi i_{A i}}} \exp \left(-\lambda^{\alpha_{1} \zeta}\right) W^{h}(z, t, \lambda, \zeta) d \zeta\right)\right. \\
& \left.+\left(\int_{\lambda^{\alpha_{i}-\alpha_{1_{B_{\imath}}}}}+\int_{\lambda^{\alpha_{i}-\alpha_{1} B_{i}^{\prime \prime}}} \exp \left(-\lambda^{\alpha_{1} \zeta}\right) W^{n}(z, t, \lambda, \zeta) d \zeta\right)\right\} \\
& +\int_{C_{p}} \exp \left(-\lambda^{\alpha_{1} \zeta}\right) W^{h}(z, t, \lambda, \zeta) d \zeta,
\end{aligned}
$$

where if $h \neq m$ the last term integrated on $C_{p}$ does not appear.

Proof. We have

$$
\begin{aligned}
& \hat{K}^{h}(z, t, \lambda)=\int_{C_{0}(-\pi)} \exp \left(-\lambda^{\alpha_{1} \zeta}\right) W^{h}(z, t, \lambda, \zeta) d \zeta \\
& =\left(\int_{A_{0}}+\int_{\lambda^{1-\alpha_{1} B_{0}}}+\int_{-e^{2 \pi i_{A_{0}}}}\right) \exp \left(-\lambda^{a_{1} \zeta}\right) W^{h}(z, t, \lambda, \zeta) d \zeta \\
& =\left(\int_{A_{0}}+\int_{\lambda^{1-\alpha_{1_{B}}^{\prime}}}+\int_{C_{1}}+\int_{\lambda^{1-\alpha_{1_{B}}^{\prime \prime}}}+\int_{-e^{2 \pi i_{A_{0}}}}\right) \exp \left(-\lambda^{\alpha_{1}} \zeta\right) W^{h}(z, t, \lambda, \zeta) d \zeta .
\end{aligned}
$$

Since

$$
\int_{C_{1}} \exp \left(-\lambda^{\alpha_{1}} \zeta\right) W^{h}(z, t, \lambda, \zeta) d \zeta=\left(\int_{B_{1}^{\prime}}+\int_{C_{2}}+\int_{B_{1}^{\prime \prime}}\right) \exp \left(-\lambda^{\alpha_{1} \zeta}\right) W^{h}(z, t, \lambda, \zeta) d \zeta
$$

and 


$$
\begin{aligned}
& \int_{C_{i}} \exp \left(-\lambda^{\alpha_{1}} \zeta\right) W^{h}(z, t, \lambda, \zeta) d \zeta \\
& =\left(\int_{\lambda} \alpha_{i-\alpha_{1} B_{i}^{\prime}}+\int_{C_{i+1}}+\int_{\lambda \alpha_{i}-\alpha_{B_{i}^{\prime \prime}}}\right) \exp \left(-\lambda^{\alpha_{1}} \zeta\right) W^{h}(z, t, \lambda, \zeta) d \zeta,
\end{aligned}
$$

we have (6.67). If $h \neq m, W^{h}(z, t, \lambda, \zeta)$ is holomorphic at $\zeta=0$, so the last term in (6.67) vanishes.

For our purpose we further decompose $\hat{K}^{h}(z, t, \lambda)$. In order to do so we need several lemmas concerning the paths $A_{i}, B_{i}^{\prime}$ and $B_{i}^{\prime \prime}$. The following lemmas are the same as in [12] and the proofs are not so difficult. So we omit them.

Lemma 6.22. Let $\zeta \in A_{i}(0 \leqq i \leqq p-1)$ and $\arg \lambda=\pi$. Then there is a $c>0$ such that $\operatorname{Re} \lambda^{a_{1}} \zeta \geqq c|\lambda|^{\alpha_{i}}$.

Lemma 6.23. Let $K$ be a compact set in $C^{1}$ and $K \cap \bar{S}_{i}=\varnothing$. If the diameter of $K$ is sufficiently small, then there are $c_{K}>0$ and $\phi_{K}$ with $\left|\phi_{k}-\pi\right|<\pi / 2 \alpha_{i}$ such that $\operatorname{Re} \lambda^{\alpha_{1}} \zeta \geqq c_{K}|\lambda|^{a_{i}}$ for $\zeta \in \lambda^{a_{i}-\alpha_{1}} K$ and $\lambda$ with $\arg \lambda=\phi_{K}$.

By Lemma 6.23 we can decompose the paths $B_{i}^{\prime}$ and $B_{\imath}^{\prime \prime}$ in the following way.

Proposition 6.24. There are paths $B_{i, s}\left(1 \leqq s \leqq r_{i}\right)$, constants $\phi_{i, s}$ with $\left|\phi_{i, s}-\pi\right|$ $<\pi / 2 \alpha_{i}$ and $c>0$, which are all independent of $\lambda$ such that $B_{i}^{\prime}=\sum_{s=1}^{r_{i}^{\prime}} B_{i, s}$ and $B_{\imath}^{\prime \prime}=\sum_{s=r_{i}^{\prime}{ }^{\prime}}^{r_{i}} B_{i, s}$, and $\operatorname{Re} \lambda^{\alpha_{1}} \zeta \geqq c|\lambda|^{\alpha_{i}}$ for $\zeta \in \lambda^{a_{i-\alpha_{1}}} B_{i, s}$ and $\lambda$ with $\arg \lambda=\psi_{i, s}$.

Define, by using $A_{i}(0 \leqq i \leqq p-1), B_{i, 。}$ in Proposition 6.24 and $C_{p}$,

$$
\begin{aligned}
& \hat{K}_{i, 0}^{h}(z, t, \lambda)=\left(\int_{A_{i}}+\int_{-e^{2 \pi i_{A i}}}\right) \exp \left(-\lambda^{\alpha_{1} \zeta}\right) W^{h}(z, t, \lambda, \zeta) d \zeta, \\
& \hat{K}_{i, s}^{h}(z, t, \lambda)=\int_{B_{i, s}(\lambda)} \exp \left(-\lambda^{\alpha_{1} \zeta}\right) W^{h}(z, t, \lambda, \zeta) d \zeta, \quad B_{i, s}(\lambda)=\lambda^{\alpha_{i}-a_{1}} B_{i, s}
\end{aligned}
$$

and

$$
\hat{K}_{p, 0}^{h}(z, t, \lambda)=\int_{C_{p}} \exp \left(-\lambda^{\alpha}{ }^{1} \zeta\right) W^{h}(z, t, \lambda, \zeta) d \zeta
$$

Then we have

$$
\hat{K}^{h}(z, t, \lambda)=\sum_{i=0}^{p} \sum_{s=0}^{r_{i}} \hat{K}_{i, s}^{h}(z, t, \lambda),
$$

where $r_{p}=0$ and $\hat{K}_{p, 0}^{h}(z, t, \lambda)=0(h \neq m) . \quad \hat{K}_{i, s}^{h}(z, t, \lambda) \quad\left(0 \leqq i \leqq p, 0 \leqq s \leqq r_{i}\right)$ are holomorphic on $\tilde{\Lambda}_{0}^{*}, \Lambda_{0}^{*}=\left\{\lambda ;|\lambda| \geqq \Lambda_{0}\right\}$, as functions of $\lambda$ and holomorphic on $X^{\prime}$ as functions of $(z, t)$. As for the estimates of them we have 
Proposition 6.25. The following estimates hold for $(z, t, \lambda) \in \tilde{X}_{\Lambda_{0}}^{\prime}$ :

(1) for each $\hat{K}_{i, 0}^{h}(z, t, \lambda)(0 \leqq i \leqq p-1)$

$$
\left|\hat{K}_{i, 0}^{h}(z, t, \lambda)\right| \leqq A \exp \left(C|\lambda|^{\alpha_{i-1}}+\left(\sin \delta_{0}\right)\left|\lambda t_{0}\right|\right) \text { on } \tilde{\Lambda}_{0}^{*},
$$

$$
\left|\hat{K}_{i, 0}^{h}(z, t, \lambda)\right| \leqq A \exp \left(-c|\lambda|^{\alpha_{i}}+\left(\sin \delta_{0}\right)\left|\lambda t_{0}\right|\right) \quad \text { on }\left\{\lambda \in \tilde{\Lambda}_{0}^{*} ; \arg \lambda=\pi\right\}
$$

and

$$
\left|\hat{K}_{p, 0}^{m}(z, t, \lambda)\right| \leqq A \exp \left(C|\lambda|^{\alpha}{ }^{p-1}+\left(\sin \delta_{0}\right)\left|\lambda t_{0}\right|\right) \text { on } \tilde{\Lambda}_{0}^{*},
$$

(6.75) $\left|\hat{K}_{p, 0}^{m}(z, t, \lambda)\right| \leqq A(1+|\lambda|)^{N} \exp \left(\left(\sin \delta_{0}\right)\left|\lambda t_{0}\right|\right)$ on $\left\{\lambda \in \tilde{\Lambda}_{0}^{*} ; \arg \lambda=\pi\right\}$.

(2) for each $\hat{K}_{i, s}^{h}(z, t, \lambda)(0 \leqq i \leqq p-1, s \neq 0)$

$$
\left|\hat{K}_{i, s}^{h}(z, t, \lambda)\right| \leqq A \exp \left(C|\lambda|^{\alpha_{i}}+\left(\sin \delta_{0}\right)\left|\lambda t_{0}\right|\right) \text { on } \tilde{\Lambda}_{0}^{*},
$$

where $\delta_{0}=\sin ^{-1}\left(c^{*} B\right)$ and all constants are positive.

Proof. The estimates (6.72), (6.74) and (6.76) are obvious. The estimates (6.73) and (6.77) follow from Proposition 6.24. It remains to show (6.75). It follows from Proposition 6.11-(2) that

$$
\begin{aligned}
\hat{K}_{p, 0}^{m}(z, t, \lambda) & =\int_{C_{p}} \exp \left(-\lambda^{\alpha_{1}} \zeta\right) W^{m}(z, t, \lambda, \zeta) d \zeta \\
& =\frac{1}{2 \pi i} \int_{C_{p}} \exp \left(-\lambda^{\alpha_{1} \zeta}\right)\left\{\frac{a(\zeta, \lambda)}{\zeta}+b(\zeta, \lambda) \log \zeta\right\} d \zeta \\
& =a(0, \lambda)+\int_{0}^{a_{p-1} e^{-i \pi \alpha_{p-1} \alpha_{p-1-\alpha_{1}}}} \exp \left(-\lambda^{\alpha_{1} \zeta}\right) b(\zeta, \lambda) d \zeta,
\end{aligned}
$$

where we deform $C_{p}$ to the path $C_{p}^{\prime}, C_{p}^{\prime}=\left\{\zeta(s)=(1-2 s) d_{p-1} e^{-i \pi a_{p-1}} \lambda^{\alpha} p-1^{-a_{1}}\right.$ $\left.(0 \leqq s \leqq 1 / 2), \zeta(s)=(2 s-1) d_{p-1} e^{-i \pi \alpha_{p-1}+2 \pi i} \lambda^{\alpha} p-1^{-\alpha_{1}} \quad(1 / 2 \leqq s \leqq 1)\right\}$, and use

$$
\frac{1}{2 \pi i} \int_{c_{p}^{\prime}} \exp \left(-\lambda^{\alpha_{1}} \zeta\right) b(\zeta, \lambda) \log \zeta d \zeta=\int_{0}^{a_{p-1} e^{-i \pi \alpha_{p-1} \alpha^{\alpha}{ }^{2}-\alpha_{1}}} \exp \left(-\lambda^{a_{1}} \zeta\right) b(\zeta, \lambda) d \zeta .
$$

If $|\arg \lambda-\pi|<\pi / 2 \alpha_{p-1},\left|\exp \left(-\lambda^{\alpha_{1}} \zeta\right)\right|$ is bounded on $C_{p}^{\prime}$. Thus we have (6.75).

We divide $u_{-\pi}^{h}(z)$, by using $\hat{K}_{i, s}^{h}(z, t, \lambda)$, into the sum of $u_{-\pi, i, s}^{h}(z)$. Put

$$
\begin{gathered}
K_{i, s}^{h}(w, z, t)=\int_{\Lambda_{0}}^{\infty e^{i \psi}} \exp (-\lambda w) \hat{K}_{i, s}^{h}(z, t, \lambda) d \lambda, \\
u_{-\pi, i, s}^{h}(z)=\int_{T_{0} \times T^{\prime \prime}} K_{i, s}^{h}\left(t_{0}-z_{0}, z, t^{\prime \prime}\right) u^{h}\left(t_{0}, 0, t^{\prime \prime}\right) d t_{0} d t^{\prime \prime}
\end{gathered}
$$

for $0 \leqq h \leqq m-1$ and

$$
u_{-\pi, i, s}^{m}(z)=\int_{T_{0} \times T^{\prime}} K_{i, s}^{m}\left(t_{0}-z_{0}, z, t\right) f(t) d t .
$$


Thus we have

$$
u(z)=\sum_{(h, i, s)} u_{-\pi, i, s}^{h}(z)+v(z), \quad v(z) \in \mathcal{O}(U),
$$

where $U=U_{0} \times U^{\prime}, U_{0}=\left\{z_{0} \in C^{1} ;\left|z_{0}\right| \leqq r\right\}$ and $U^{\prime}=\left\{z^{\prime} \in C^{n} ;\left|z_{1}-\hat{z}_{1}\right|<\varepsilon_{1},\left|z^{\prime}\right| \leqq r\right\}$.

In the rest of this section $U$ means that defined above, where $r$ is small if necessary, and we consider a solution $u(z)$ of $(6.1)$ and $f(z)$ satisfying some growth conditions:

$$
\left\{\begin{array}{l}
u(z) \in \mathcal{O}_{(\gamma), h^{\prime}}\left(\Omega\left(\theta_{0}\right)\right), \quad \gamma=\sigma_{p-1}-1, \\
L\left(z, \partial_{z}\right) u(z)=f(z) \in A s y_{(\gamma)}\left(\Omega\left(\theta_{0}\right)\right),
\end{array}\right.
$$

where $\theta_{0}>\pi / 2+\varepsilon_{0}, \varepsilon_{0}>\delta_{0}=\sin ^{-1}\left(c^{*} B\right)$, and $L\left(z, \partial_{z}\right)$ satisfies (6.2). From (6.83)

$$
\left|\partial_{1}^{k} u\left(t_{0}, 0, t^{\prime \prime}\right)\right| \leqq A \exp \left(h^{\prime}\left|t_{0}\right|^{-\gamma}\right)
$$

Proposition 6.26. Assume (6.2), (6.4) and (6.83).

(1) $u_{-\pi, i, s}^{h}(z)(i \neq 0,(i, s) \neq(1,0))$ are holomorphically extensible to $U\left(\theta_{0}-\varepsilon_{0}\right)$ and

$$
\begin{cases}\left|u_{-\pi, i, s}^{h}(z)\right| \leqq A_{\theta}, \exp \left(c_{\theta},\left|z_{0}\right|^{-r_{2}}\right), & s \neq 0, \\ \left|u_{-\pi, i, 0}^{h}(z)\right| \leqq A_{\theta}, \exp \left(c_{\theta},\left|z_{0}\right|^{-r_{1-1}}\right), & i \neq 1,\end{cases}
$$

in $z \in U\left(\theta^{\prime}\right)$ with any $\theta^{\prime}$ with $\theta^{\prime}<\theta_{0}-\varepsilon_{0}$, where $\gamma_{i}=\sigma_{i}-1=\alpha_{i} /\left(1-\alpha_{i}\right)$.

(2) $u_{-\pi, i, s}^{h}(z)(i=0$ or $(i, s)=(1,0))$ are holomorphic at the origin.

Proof. (1) It follows from (6.72), (6.74) and (6.76) that if $i \neq 0$ or $(i, s) \neq$ $(1,0),\left|\hat{K}_{i, s}^{h}(z, t, \lambda)\right| \leqq A \exp \left(C|\lambda|^{a}+\left(\sin \delta_{0}\right)\left|\lambda t_{0}\right|\right)$ for some $0<\alpha<1$ on $\tilde{\Lambda}_{0}^{*}$. So we have the assertion from $(6.83)^{\prime}$ and Proposition 5.2-(1).

(2) Suppose $i=0$ or $(i, s)=(1,0)$. By $(6.73) \quad\left|\hat{K}^{h}(z, t, \lambda)\right| \leqq A \exp (-c|\lambda|+$ $\left.\left(\sin \delta_{0}\right)\left|\lambda t_{0}\right|\right)$ on $\left\{\lambda \in \tilde{\Lambda}_{0}^{*} ; \arg \lambda=\pi\right\}$. So $K_{i, s}^{h}(w, z, t)$ is holomorphic at $w=0$. This means that $u_{-\pi, i, s}^{h}(z)$ is holomorphic at $z=0$.

Now we use the estimates (6.73), (6.75) and (6.77) on the line $\left\{\lambda ; \arg \lambda=\phi_{i, s}\right\}$, where $\phi_{i, 0}=\pi$. Put

$$
\hat{\theta}=\frac{\pi}{2}+\max \left\{\left|\phi_{i, s}-\pi\right|\right\}<\frac{\pi}{2}+\frac{\pi}{2 \alpha_{p-1}}=\frac{\pi}{2 \gamma}+\pi .
$$

We obtain the asymptotic expansion of $u_{-\pi, i, s}^{h}(z)$.

Proposition 6.27. Assume the same conditions as in Proposition 6.26 and $\theta_{0}>\hat{\theta}+\varepsilon_{0}, \varepsilon_{0}>\delta_{0}$. Then $u_{-\pi, i, s}^{h}(z)(i \neq p-1)$ have the $\gamma_{i}$-asymptotic expansion with respect to $z_{0}$ with $\left\{z_{0} ;\left|\arg z_{0}+\psi_{i, s}-\pi\right|<\pi / 2\right\}$ in $U$. If $h^{\prime}$ is small, $u_{-\pi, p-1, s}^{h}(z)$ have the $\gamma_{p-1}$-asymptotic expansion with respect to $z_{0}$ with $\left\{z_{0} ;\left|\arg z_{0}+\phi_{i, s}-\pi\right|\right.$ $<\pi / 2\}$ in $U$.

Proof. We apply Proposition 5.2 to $u_{-\pi, i, s}^{h}(z)$. The bounds (6.73) or (6.77) 
holds for $(h, \imath, s) \neq(m, p, 0)$. By the assumptions $\left(-\psi_{i, s}+(\pi / 2)-\varepsilon_{0},-\phi_{i, s}+\right.$ $\left.(3 \pi / 2)-\varepsilon_{0}\right) \subset\left(-\theta_{0}, \theta_{0}\right)$. So we have the $\gamma_{i}$-asymptotic expansion by Proposition 5.2-(2). Since $\alpha_{i}>\alpha_{p-1}(i \neq p-1), h^{\prime}$ is not necessarily small for $i \neq p-1$. For $(h, i, s)=(m, p, 0)$ the bounds (6.75) holds. In this case we have the $\gamma_{p-1^{-}}$ asymptotic by Proposition 5.2-(3).

We note that since $\gamma=\gamma_{p-1} \leqq \gamma_{i}$, we can say that there is an $h_{0}^{\prime}$ such that if $0<h^{\prime}<h_{0}^{\prime}$ in (6.83), then $u_{-\pi, i, s}^{h}(z)$ has the $\gamma$-asymptotic expansion with respect to $z_{0}$ in $\left\{\pi / 2<\arg z_{0}+\phi_{i, s}<3 \pi / 2\right\}$. We note that from the condition $\left|\phi_{i, s}-\pi\right|$ $<\pi / 2 \alpha_{i}$ there are $\varphi_{i, s}$ and $\kappa_{i}$ such that $\left|\varphi_{i, s}\right|<\pi / 2 \kappa_{i}<\pi / 2 \gamma_{i}$ and $u_{-\pi, i, s}^{h}(z)$ has the $\gamma$-asymptotic expansion with respect to $z_{0}$ on $\arg z_{0}=\varphi_{i, s}$. Thus, by using Propositions 2.8 and 2.10 , we have

Theorem 6.28. Assume (6.2). Let $u(z) \in \mathcal{O}_{(1), h^{\prime}}\left(\Omega\left(\theta_{0}\right)\right), \gamma=\sigma_{p-1}-1$, be a solution of $L\left(z, \partial_{z}\right) u(z)=f(z) \in A s y_{(r)}\left(\Omega\left(\theta_{0}\right)\right)$. Then there are positive constants $H$ and $\Theta$ with $0<\Theta<\pi / 2 \gamma+\pi$ such that, if $\theta_{0}>\Theta$ and $h^{\prime}<H, u(z) \in A s y_{(\gamma)}\left(\Omega\left(\theta^{\prime}\right)\right)$, where $\theta^{\prime}=\theta^{\prime}\left(h^{\prime}\right)$ with $\lim _{h^{\prime} \rightarrow 0} \theta^{\prime}\left(h^{\prime}\right)=\theta_{0}$.

Proof. It follows from the assumption (6.2) that (1.14) holds. So we may assume (1.14) holds at $z^{\prime}=0$ and $\hat{\xi}^{\prime}=(1,0, \cdots, 0)$, that is, (6.4) holds. Put $\Theta=$ $(\hat{\theta}+\pi / 2 \gamma+\pi) / 2$. Obviously $\pi / 2 \gamma+\pi>\Theta>\hat{\theta}$ by (6.85). Choose $\delta_{0}$ and $\varepsilon_{0}$ with $0<\delta_{0}<\varepsilon_{0}<\Theta-\hat{\theta}$ and fix them. Suppose $\theta_{0}>\Theta>\hat{\theta}+\varepsilon_{0}$. Then it follows from Proposition 6.27 that if $0<h^{\prime}<h_{0}^{\prime}, u_{-\pi, i, s}^{h}(z)$ has the $\gamma$-asymptotic expansion with respect to $z_{0}$ on $\left\{\arg z_{0}=\varphi_{i, s}\right\}$ in an open set $U, \Omega \supset U \neq \varnothing$. It follows from Proposition 2.10 that if $0<h^{\prime}<\min \left(h_{0}^{\prime}, h_{0}\right)=H, h_{0}$ being that in Proposition 2.10, $u(z)$ has the $\gamma$-asymptotic expansion in $U\left(\theta^{\prime}\right)$ for some $\theta^{\prime}=\theta^{\prime}\left(h^{\prime}\right)$ with $\lim _{h^{\prime} \rightarrow 0} \theta^{\prime}\left(h^{\prime}\right)$ $=\theta_{0}$. Hence, from Proposition 2.8, $u(z)$ has the $\gamma$-asymptotic expansion in $\Omega\left(\theta^{\prime}\right)$.

\section{§7. Proof of Theorems and Estimates}

In $\S 7$ we give the proof of Theorems $1.13,1.5$ and 1.7 , and finally show Propositions 4.1 and 6.8 which concern with estimates of functions and are not yet shown. For these purposes the method of majorant power series is available. So we summerize what we need. Let $A(z)=\sum A_{\alpha} z^{\alpha}$ and $B(z)=\sum B_{a} z^{\alpha}$ be formal power series, where $\alpha=\left(\alpha_{0}, \alpha_{1}, \cdots, \alpha_{n}\right)=\left(\alpha_{0}, \alpha^{\prime}\right) \in Z_{+}^{n+1}$. Then $A(z)$ $\gg 0$ means $A_{\alpha} \geqq 0$ and $A(z) \ll B(z)$ means $\left|A_{\alpha}\right| \leqq B_{a}$ for all multi-indices $\alpha$. We state elementary properties of majorant power series without the proof, which will be often used. For the proof we refer to [2], [5] and [15].

Lemma 7.1 (Wagschal). Let $\Theta(s)$ be a formal power series of one variable $s$ such that $\Theta(s) \gg 0$ and 


$$
\left(R^{\prime}-s\right) \Theta(s) \gg 0 .
$$

Then for derivatives $\Theta^{(j)}(s)=(d / d s)^{j} \Theta(s)(j=0,1, \cdots)$ we have

$$
\left(R^{\prime}-s\right) \Theta^{(j)}(s) \gg 0, \quad R^{\prime} \Theta^{(j+1)}(s) \gg \Theta^{(j)}(s)
$$

and

$$
\left(R_{0}-s\right)^{-1} \Theta^{(\jmath)}(s) \ll\left(R_{0}-R^{\prime}\right)^{-1} \Theta^{(j)}(s) \quad\left(R_{0}>R^{\prime}\right) .
$$

Lemma 7.2 (Wagschal). Let $\Theta(s)$ be a formal power series of one variable $s$ such that $\Theta(s) \gg 0$ and $\left(R^{\prime}-s\right) \Theta(s) \gg 0$. Let $M\left(z, \partial_{z}\right)$ be a linear partial differential operator of order $m$ with the coefficients holomorphic in $\left\{|z| \leqq R_{0}\right\}, R^{\prime}$ $<R_{0}$. Then

$$
M\left(z, \partial_{z}\right) \Theta(s) \ll A \Theta^{(m)}(s), \quad s=z_{0}+z_{1}+\cdots+z_{n},
$$

for a constant $A$ which is independent of $\Theta(s)$.

Now we proceed to show Theorem 1.13. Firstly we have

Proposition 7.3. Assume $L\left(z, \partial_{z}\right)$ satisfies the conditions (1.17)-(a), (b), (c) and put $\gamma=\sigma_{p-1}-1$. Let $f(z) \in A s y_{(\kappa)}\left(\Omega\left(\theta_{0}\right)\right)$ with $0<\kappa \leqq \gamma$ and $0<\theta_{0} \leqq \pi / 2 \kappa$. Then there exists a function $u(z) \in A s y_{[\kappa\}}\left(\Omega\left(\theta_{0}\right)\right)$ such that $\left(L\left(z, \partial_{z}\right) u(z)-f(z)\right) \sim 0$ as a function in $A s y_{[\kappa]}\left(\Omega\left(\theta_{0}\right)\right)$.

Proof. From Remark 1.12, $L\left(z, \partial_{z}\right)$ is written in the form

$$
L\left(z, \partial_{z}\right)=a_{k p-1,0}(z)\left(\partial_{0}\right)^{k} p-1+\sum_{(k, l) \neq(k p-1,0)} z_{0}^{j(k, l)} a_{k, l}\left(z, \partial^{\prime}\right)\left(\partial_{0}\right)^{k-l},
$$

where $k-d_{k, l} \leqq k_{p-1}$. Let $f(z) \sim \sum_{n=0}^{\infty} f_{n}\left(z^{\prime}\right)\left(z_{0}\right)^{n} / n !, f_{n}\left(z^{\prime}\right) \in \mathcal{O}\left(\Omega^{\prime}\right)$, and $u(z) \sim$ $\sum_{n=k}^{\infty} u_{n-1}\left(z^{\prime}\right)\left(z_{0}\right)^{n} / n !$. Then $u_{n}\left(z^{\prime}\right)\left(n \geqq k_{p-1}\right)$ are determined by

$$
\begin{aligned}
a_{k_{p-1}, 0}^{0}\left(z^{\prime}\right) u_{n}\left(z^{\prime}\right)= & -\sum_{\substack{j+r-k+l=n-k_{p-1} \\
\left(k_{,}, j\right) \geq(k p-1,0,0)}} \frac{\left(n-k_{p-1}\right) !}{\left(n-k_{p-1}-j\right) !} a_{k, l}^{j}\left(z^{\prime}, \partial^{\prime}\right) u_{r}\left(z^{\prime}\right) \\
& +f_{n-k_{p-1}}\left(z^{\prime}\right) .
\end{aligned}
$$

We show, by induction,

$$
u_{n}\left(z^{\prime}\right) \ll A B^{n} \theta^{[(n-k p-1) / k]+n-k} p-1(s),
$$

where $\theta(s)=\left(R^{\prime}-s\right)^{-1}, s=z_{1}+z_{2}+\cdots+z_{n}$.

By the assumption $f_{n}\left(z^{\prime}\right) \ll A B^{n} \theta^{[n / k]+n}$. So (7.7) is obvious for $n=k_{p-1}$. Assume (7.7) holds for $r$ with $r<n$. Then

$$
\begin{aligned}
& \frac{\left(n-k_{p-1}\right) !}{\left(n-k_{p-1}-j\right) !} a_{k, l}^{j}\left(z^{\prime}, \partial^{\prime}\right) u_{r}\left(z^{\prime}\right) \\
& \ll A M B^{r} C^{j} \frac{\left(n-k_{p-1}\right) !}{\left(n-k_{p-1}-j\right) !} \theta^{\left[\left(r-k_{p-1}\right) / k\right]+r-k_{p-1}+l}(s) .
\end{aligned}
$$


Since $l+j \geqq \sigma_{p-1}\left(k-k_{p-1}\right)=(\gamma+1)\left(k-k_{p-1}\right) \geqq(\kappa+1)\left(k-k_{p-1}\right)$ for $k \geqq k_{p-1},[(r-$ $\left.\left.k_{p-1}\right) / \kappa\right]=\left[\left(n-2 k_{p-1}+k-l-j\right) / \kappa\right] \leqq\left[\left(n-k_{p-1}\right) / \kappa\right]+k_{p-1}-k$. Hence

$$
\begin{aligned}
& \frac{\left(n-k_{p-1}\right) !}{\left(n-k_{p-1}-j\right) !} a_{k, l}^{j}\left(z^{\prime}, \partial^{\prime}\right) u_{\tau}\left(z^{\prime}\right) \\
& \ll A M B^{r} C^{j} n^{j} \theta^{[(n-k p-1) / k]+r+l-k}(s) \\
& \left.\left.\ll A M B^{r} D^{j} \theta^{[(n-k} p-1\right) / k\right]+r+l-k+j(s) \\
& \left.\left.\ll A M B^{r} D^{j} \theta^{[(n-k} p-1\right) / \kappa\right]+n-k p-1(s) .
\end{aligned}
$$

Thus we have (7.7). Since $0<\theta_{0} \leqq \pi / 2 \kappa$, it follows from Proposition 2.1 that there is a $u(z) \in A s y_{(\kappa)}(U(\pi / 2 \kappa))$ in a neighbourhood $U$ such that $u(z) \sim \sum_{n=k}^{\infty}{ }_{p-1}$ $u_{n}\left(z^{\prime}\right)\left(z_{0}\right)^{n}$ and $\left(L\left(z, \partial_{z}\right) u(z)-f(z)\right) \sim 0$ in $A s y_{(\kappa)}\left(U\left(\theta_{0}\right)\right)$.

Proof of Theorem 1.13. It follows from Proposition 7.3 that there is a $u(z) \in A s y_{(x)}\left(\Omega\left(\theta_{0}\right)\right)$ such that $g(z)=\left(L\left(z, \partial_{z}\right) u(z)-f(z)\right) \sim 0$ as a function in $A s y_{(\kappa)}\left(\Omega\left(\theta_{0}\right)\right)$. Define, as in Proposition 2.13,

$$
\tilde{u}(z)=\int_{0}^{r} \frac{u\left(t_{0}, z^{\prime}\right)}{z_{0}-t_{0}} d t_{0} .
$$

We have, by integrations by parts, for multi-index $\alpha$ with $\alpha_{0} \leqq k_{p-1}$

$$
\begin{aligned}
\partial_{z}^{\alpha} \tilde{u}(z) & =(-1)^{\alpha_{0}} \alpha_{0} ! \int_{0}^{r}\left\{\partial_{z}^{\alpha^{\prime}} u\left(t_{0}, z^{\prime}\right) /\left(z_{0}-t_{0}\right)^{\alpha_{0}+1}\right\} d t_{0} \\
& =\int_{0}^{r}\left\{\left(\partial_{t_{0}}\right)^{\alpha} \partial_{z}^{\alpha^{\prime}} u\left(t_{0}, z^{\prime}\right) /\left(z_{0}-t_{0}\right)\right\} d t_{0}+g_{1}(z),
\end{aligned}
$$

where $g_{1}(z) \in \mathcal{O}(U), U(|z|<r)$, is determined by the values of the derivatives of $u\left(t_{0}, z^{\prime}\right)$ at $t_{0}=r$. Let $A(z)$ be holomorphic in $Q$ and put

$$
g_{2}(z)=\int_{0}^{r}\left(A\left(z_{0}, z^{\prime}\right)-A\left(t_{0}, z^{\prime}\right)\right)\left\{\left(\partial_{t_{0}}\right)^{\alpha} \partial_{z^{\prime}}^{\alpha^{\prime}} u\left(t_{0}, z^{\prime}\right) /\left(z_{0}-t_{0}\right)\right\} d t_{0} .
$$

Then we have

$$
g_{2}(z)=\int_{0}^{r}\left\{\int_{0}^{1}\left(\partial_{0} A\left(s z_{0}+(1-s) t_{0}, z^{\prime}\right) d s\right\}\left(\partial_{t_{0}}\right)^{\alpha_{0}} \partial_{z^{\prime}}^{\alpha^{\prime}} u\left(t_{0}, z^{\prime}\right) d t_{0}\right.
$$

and $g_{2}(z) \in \mathcal{O}(U)$. Hence we have

$$
A(z) \partial^{\alpha} \tilde{u}(z)=\int_{0}^{r} A\left(t_{0}, z^{\prime}\right)\left\{\left(\partial_{t_{0}}\right)^{\alpha_{0}} \partial_{z^{\prime}}^{\alpha^{\prime}} u\left(t_{0}, z^{\prime}\right) /\left(z_{0}-t_{0}\right)\right\} d t+g_{3}(z),
$$

where $g_{3}(z) \in \mathcal{O}(U)$. Thus there is a $g_{0}(z) \in \mathcal{O}(U)$ such that

$$
\begin{aligned}
& L\left(z, \partial_{z}\right) \tilde{u}(z)=\int_{0}^{r}\left\{L\left(t_{0}, z^{\prime}, \partial_{t_{0}}, \partial_{z^{\prime}}\right) u\left(t_{0}, z^{\prime}\right) /\left(z_{0}-t_{0}\right)\right\} d t_{0}+g_{0}(z) \\
& =\int_{0}^{r}\left(f\left(t_{0}, z^{\prime}\right) /\left(z_{0}-t_{0}\right)\right) d t_{0}+\int_{0}^{r}\left(g\left(t_{0}, z^{\prime}\right) /\left(z_{0}-t_{0}\right)\right) d t_{0}+g_{0}(z)=\tilde{f}(z)+\tilde{g}(z)+g_{0}(z) .
\end{aligned}
$$


It follows from the proof of Proposition 2.13 that $\tilde{f}(z)-f(z) \log z_{0}, \tilde{g}(z)-g(z) \log z_{0}$ $\in A s y_{(\kappa)}\left(U\left(\theta_{0}\right)\right)$. Since $g(z) \sim 0$ in $A s y_{(\kappa)}\left(U\left(\theta_{0}\right)\right), \tilde{g}(z) \in A s y_{(\kappa)}\left(U\left(\theta_{0}\right)\right)$. This means $L\left(z, \partial_{z}\right) \tilde{u}(z)-f(z) \log z_{0}=\tilde{f}(z)-f(z) \log z_{0}+\tilde{g}(z)+g_{0}(z) \in A s y_{(x)}\left(U\left(\theta_{0}\right)\right)$.

Now we show Theorems 1.5 and 1.7. Let $u(z) \in \mathcal{O}_{(\gamma)}\left(\Omega\left(\theta_{0}\right)\right)$ be a solution of

$$
L\left(z, \partial_{z}\right) u(z)=f(z) .
$$

Proof of Theorem 1.5. We use Theorems 1.11 and 6.28. The positive constants $H$ and $\Theta$ are those in Theorem 6.28. Suppose that $f(z) \in A s y_{(\kappa)}\left(\Omega\left(\theta_{0}\right)\right)$ in (7.16). Sincce $d_{k_{p-1}}=0, a_{k_{p-1}, 0}\left(0, z^{\prime}\right) \not \equiv 0$. We may assume that $a_{k_{p-1}, 0}(0,0)$ $\neq 0$, that is, (1.17)-(c) holds and $\theta_{0}<\pi / 2 \gamma+\pi=\pi / 2 \alpha_{p-1}+\pi / 2$. By Proposition 2.11 , we can decompose $u(z):$ for $h>0$

$$
u(z)=\sum_{i=1}^{l} u_{i}(z), \quad u_{i}(z) \in \mathcal{O}_{(\gamma), h}\left(U\left(a_{i}, b_{i}\right)\right),
$$

where $U$ is a polydisk with the center $z=0,-\left(\pi / \alpha_{p-1}+\pi\right)<a_{i}<0<b_{i}<\pi / \alpha_{p-1}$ $+\pi$ and $2 \Theta<b_{i}-a_{i}$. Put $I_{i}=\left(a_{i}, b_{i}\right)$. We have $\bigcap_{i=1}^{l} I_{i}=I_{0}=\left(-\theta_{0}, \theta_{0}\right)$. Take $\delta_{0}$ and $\varepsilon$ so that $\left(b_{i}-a_{i}\right) / 2-\Theta>\varepsilon>\delta_{0}$ for all $i$. Put

$$
f_{i}(z)=L\left(z, \partial_{z}\right) u_{i}(z) \in \mathcal{O}_{[\gamma], h}\left(U\left(a_{\imath}, b_{i}\right)\right) \text { and } \phi_{i}=\left(a_{i}+b_{i}\right) / 2-\pi(i>0) .
$$

Define $v_{i}(z)=\left(G^{\dot{\varphi}_{2}} f_{i}\right)(z)(i \geqq 1)$. By Theorem 1.11-(1) and (2)

$$
L\left(z, \partial_{z}\right) v_{i}(z) \equiv f_{i}(z)+\left(G_{R} \psi_{i} f_{i}\right)(z),
$$

where $v_{i}(z) \in \mathcal{O}_{(r), c h}\left(U\left(a_{i}+\varepsilon, b_{i}-\varepsilon\right)\right), c=c(\varepsilon) \geqq 1$. If $h<h_{1}, h_{1}$ being that in Theorem 1.11-(5), $\left(G_{R}^{\psi^{i}} f_{i}\right)(z) \in A s y_{(\gamma)}\left(U\left(a_{i}+\delta_{0}, b_{i}-\delta_{0}\right)\right)$. Put $w_{i}(z)=u_{i}(z)-v_{i}(z)$. Then $w_{i}(z) \in \mathcal{O}_{(r), c h}\left(U\left(a_{i}+\varepsilon, b_{\imath}-\varepsilon\right)\right)$ and

$$
L\left(z, \partial_{z}\right) w_{i}(z) \equiv-\left(G_{R} \psi_{i} f_{i}\right)(z) .
$$

Hence if follows from Theorem 6.28 that if $h$ is small, $w_{i}(z) \in A s y_{(r)}\left(U\left(a_{i}+\varepsilon\right.\right.$, $\left.\left.b_{\imath}-\varepsilon\right)\right)$. Thus $w(z)=\sum_{i=1}^{l} w_{i}(z) \in A s y_{(r)}\left(U\left(\theta_{0}-\varepsilon\right)\right)$. Now we study $v_{i}(z)$. We have

$$
v_{i}(z)=\left(G^{\psi_{i}} f_{i}\right)(z)=\left(G^{\psi_{i},-\pi} f_{i}\right)(z)+\left(G^{-\pi} f_{i}\right)(z)
$$

and

$$
\begin{aligned}
& v(z)=\sum_{i=1}^{l} v_{i}(z)=\sum_{l=1}^{l}\left(G^{\zeta^{\prime}},-\pi f_{i}\right)(z)+\sum_{i=1}^{l}\left(G^{-\pi} f_{i}\right)(z) \\
& =\sum_{i=1}^{l}\left(G^{\psi_{i},-\pi} f_{i}\right)(z)+\left(G^{-\pi} f\right)(z) .
\end{aligned}
$$

Since $-\left(\pi / \alpha_{p-1}+\pi\right)<a_{i}<0<b_{2}<\pi / \alpha_{p-1}+\pi$, it follows from Theorem 1.11-(3) that if $h<h_{0},\left(G^{\dot{\psi}_{i},-\pi} f_{i}\right)(z) \in A s y_{(\gamma)}\left(U\left(\theta_{0}-\delta_{0}\right)\right)$ for all $i$. By Theorem 1.11-(4). $f(z) \in A s y_{(\kappa)}\left(U\left(\theta_{0}\right)\right)$ implies $\left(G^{-\pi} f\right)(z) \in A s y_{(\kappa)}\left(U\left(\theta^{\prime}-\delta_{0}\right)\right), \quad \theta^{\prime}=\min \left(\theta_{0}, \pi\right)$. So $v(z)$ $\in A s y_{(\kappa)}\left(U\left(\theta^{\prime}-\delta_{0}\right)\right)$ and $u(z)=v(z)+w(z) \in A s y_{(\kappa)}\left(U\left(\theta^{\prime}-\varepsilon\right)\right)$. It follows from Proposition 2.8 that $u(z) \in A s y_{(\kappa)}\left(\Omega\left(\theta^{\prime}-\varepsilon\right)\right)$. We can choose $h, \delta_{0}$ and $\varepsilon$ arbitrary. So $u(z) \in A s y_{(\kappa)}\left(\Omega\left(\theta^{\prime}\right)\right)$. By the rotation of $z_{0}$, we have $u(z) \in A s y_{(\kappa)]}\left(\Omega\left(\theta_{0}\right)\right)$. 
Proof of Theorem 1.7. We may assume $\left|\theta_{0}\right|<\pi / 2 \kappa$. So let $f(z) \in \tilde{M}-$ $\operatorname{asy}_{(\kappa)}\left(\Omega\left(\theta_{0}\right)\right)$ with $f(z)=g(z) \log z_{0}+h(z)$, where $g(z), h(z) \in A s y_{(x)}\left(\Omega\left(\theta_{0}\right)\right)$. By Theorem 1.13 there is a $\tilde{u}(z) \in \tilde{M}-a s y_{(x)}\left(U\left(\theta_{0}\right)\right)$ such that $L\left(z, \partial_{z}\right) \tilde{u}(z)-g(z) \log z_{0}$ $\in A s y_{(\kappa)}\left(U\left(\theta_{0}\right)\right), \Omega \supset U \ni 0$. Hence $L\left(z, \partial_{z}\right)(u(z)-\tilde{u}(z)) \in A s y_{(\kappa)}\left(U\left(\theta_{0}\right)\right)$ and $(u(z)-$ $\tilde{u}(z)) \in A s y_{(\kappa)}\left(U\left(\theta_{0}\right)\right)$ by Theorem 1.5. So $u(z) \in \tilde{\mathscr{N}}-a s y_{(\kappa)}\left(U\left(\theta_{0}\right)\right)$. For general $\theta_{0}$, we have the assertion by the rotation of $z_{0}$.

Finally we show Propositions 4.1 and 6.8. In the following we assume $r<R^{\prime}<R_{0}<R_{1}<R, R_{1} \leqq\left|t_{i}\right| \leqq R(i \geqq 2)$, and $|\lambda| \geqq \Lambda_{0}$ and try to obtain estimates of holomorphic functions of $\lambda$ and $z$, considering $\tau, t$ to be parameters.

Lemma 7.4. Let $\theta(s)=\left(R^{\prime}-s\right)^{-1}$ and put $s=z_{0}+z_{1}+\cdots+z_{n}+\left(\left(\lambda-\lambda_{0}\right) /\left|\lambda_{0}-\Lambda_{0}\right|\right)$ $\left(\left|\lambda_{0}\right| \geqq 2 \Lambda_{0}\right)$. Then

$$
\begin{aligned}
& \left(\lambda t_{0}-\lambda \partial_{\lambda}\right)\left\{\sum_{r=0}^{l}\left(\left(\left|\lambda_{0}\right|+\left|\lambda_{0}-\Lambda_{0}\right|\right)\left|t_{0}\right|\right)^{r} / r !\right\} \theta^{(l)}(s) \\
& \ll A\left\{\sum_{r=0}^{l+1}\left(\left(\left|\lambda_{0}\right|+\left|\lambda_{0}-\Lambda_{0}\right|\right)\left|t_{0}\right|\right)^{r} / r !\right\} \theta^{(l+1)}(s) .
\end{aligned}
$$

Proof. We have

$$
\lambda \partial_{\lambda} \theta^{(l)}(s) \ll \frac{\left(\lambda-\lambda_{0}\right)+\left|\lambda_{0}\right|}{\left|\lambda_{0}-\Lambda_{0}\right|} \theta^{(l+1)}(s) \ll\left(1+\frac{\left|\lambda_{0}\right|}{\left|\lambda_{0}-\Lambda_{0}\right|}\right) \theta^{(l+1)}(s) \ll C \theta^{(l+1)}(s)
$$

and

$$
\begin{aligned}
\lambda t_{0} \theta^{(l)}(s) & \ll\left|t_{0}\right|\left(\left(\lambda-\lambda_{0}\right)+\left|\lambda_{0}\right|\right) \theta^{(l)}(s) \\
& \ll \frac{\left(\left(\lambda-\lambda_{0}\right)+\left|\lambda_{0}\right|\right)\left|t_{0}\right|}{l+1} \theta^{(l+1)}(s) \ll \frac{\left(\left|\lambda_{0}-\Lambda_{0}\right|+\left|\lambda_{0}\right|\right)\left|t_{0}\right|}{l+1} \theta^{(l+1)}(s),
\end{aligned}
$$

where we use $s \theta^{(l)}(s) \ll \theta^{(l)}(s)$. Hence

$$
\begin{aligned}
\left(\lambda t_{0}\right. & \left.-\lambda \partial_{\lambda}\right)\left\{\sum_{r=0}^{l}\left(\left(\left|\lambda_{0}\right|+\left|\lambda_{0}-\Lambda_{0}\right|\right)\left|t_{0}\right|\right)^{r} / r !\right\} \theta^{(l)}(s) \\
& \ll C\left\{\sum_{r=0}^{l}\left(\left(\left|\lambda_{0}\right|+\left|\lambda_{0}-\Lambda_{0}\right|\right)\left|t_{0}\right|\right)^{r} / r !\right\} \theta^{(l+1)}(s) \\
& +\left\{\sum_{r=0}^{l}\left(\left(\left|\lambda_{0}\right|+\left|\lambda_{0}-\Lambda_{0}\right|\right)\left|t_{0}\right|\right)^{r+1} /(r+1) !\right\} \theta^{(l+1)}(s) \\
& \ll A\left\{\sum_{r=0}^{l+1}\left(\left(\left|\lambda_{0}\right|+\left|\lambda_{0}-\Lambda_{0}\right|\right)\left|t_{0}\right|\right)^{r} / r !\right\} \theta^{(l+1)}(s) .
\end{aligned}
$$

Now let us write the equation in $\S 3$ :

$$
\begin{aligned}
& G_{0}^{i}(z, \lambda, \tau) v_{N}(z, t, \lambda, \tau) \\
& \quad+\Sigma_{q} G_{q}^{i}\left(z, \partial^{\prime}, \lambda, \tau\right) v_{N-q}(z, t, \lambda, \tau)=\delta_{N, n_{0}} F(z, t, \lambda),
\end{aligned}
$$

where $\Sigma_{q}$ is a finite sum and

$$
G_{q}^{i}\left(z, \partial^{\prime}, \lambda, \tau\right)=\lambda^{k_{i-1}-\left(1-a_{\imath}\right) d_{k_{i-1}}}
$$




$$
\begin{aligned}
& \left\{\sum_{\substack{k, l, s, r, d \\
s+r+d=q}}\left(\begin{array}{c}
k-l \\
r
\end{array}\right) \lambda^{-\left(1-\alpha_{\imath}\right) r-\beta_{k, l}^{l} l\left(-\alpha z_{1}\right)^{j-d} Q^{j, k}+r, d}\left(n+s+r, \lambda t_{0}-\lambda \partial_{\lambda}\right)\right. \\
& \left.\times \tau^{d_{k, l-s-d}} a_{k, l, s}\left(z, \partial^{\prime}\right) \partial_{0}{ }^{r}\right\} .
\end{aligned}
$$

$G_{0}^{i}(z, \lambda, \tau)$ is a polynomial of $\tau$ with degree $m$,

$$
\begin{aligned}
& G_{0}^{i}(z, \lambda, \tau)=\lambda^{k_{i-1}-\left(1-a_{\imath}\right) d_{k_{i-1}}}
\end{aligned}
$$

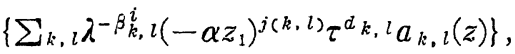

where $a_{k, l}(z)=a_{k, l}\left(z, \hat{\xi}^{\prime}\right)$ (see Lemma 3.3).

Proof of Proposition 4.1. In this case $i=p-1, d_{k_{p-1}}=0$ and $\tau=0$. Hence

$$
G_{0}(z, \lambda)=G_{0}^{p-1}(z, \lambda, 0)=\lambda^{k} p-1\left\{\sum_{(k: j(k, 0)=0,} \lambda^{-\beta_{k, 0}^{i}} a_{k, 0}(z)\right\} .
$$

We show

$$
\lambda^{k} p-1 v_{n}(z, t, \lambda) \ll A B^{n+1}\left\{\sum_{l=0}^{n+1}\left(\left(\left|\lambda_{0}\right|+\left|\lambda_{0}-\Lambda_{0}\right|\right)\left|t_{0}\right|\right)^{i} / i !\right\} \theta^{(n+1)}(s),
$$

where $\theta(s)=\left(R^{\prime}-s\right)^{-1}$ and $s=z_{0}+z_{1}+\cdots+z_{n}+\left(\left(\lambda-\lambda_{0}\right) /\left(\left|\lambda_{0}-\Lambda_{0}\right|\right)\right)$. We have $\lambda^{k}{ }^{p-1} v_{-1}(z, t, \lambda) \ll A \theta(s)$. Assume (7.27) for $-1 \leqq n \leqq N-1$. Since $a_{k, l, s}\left(z, \partial^{\prime}\right) \partial_{0}{ }^{r}$ is an operator with the order $\leqq s+r$, from Lemma 7.2 ,

$$
\begin{aligned}
& \lambda^{k p-1} a_{k, l, s}\left(z, \partial^{\prime}\right) \partial_{0}{ }^{r} v_{N-q}(z, t, \lambda) \\
& \quad \ll A B^{N-q+1}\left\{\sum_{i=0}^{N-q+1}\left(\left(\left|\lambda_{0}\right|+\left|\lambda_{0}-\Lambda_{0}\right|\right)\left|t_{0}\right|\right)^{2} / i !\right\} \theta^{(N-q+s+r+1)}(s) .
\end{aligned}
$$

It follows from Lemma 3.4, (3.15) and Lemma 7.4 that

$$
\begin{aligned}
& Q_{i+r, d}^{j, k}\left(n+s+r, \lambda t_{0}-\lambda \partial_{\lambda}\right) \\
& \quad \times\left\{\sum_{i=0}^{N-q+1}\left(\left(\left|\lambda_{0}\right|+\left|\lambda_{0}-\Lambda_{0}\right|\right)\left|t_{0}\right|\right)^{i} / i !\right\} \theta^{(N-q+s+r+1)}(s) \\
& \quad \ll A\left\{\sum_{i=0}^{N-q+s+r+d+1}\left(\left(\left|\lambda_{0}\right|+\left|\lambda_{0}-\Lambda_{0}\right|\right)\left|t_{0}\right|\right)^{i} / i !\right\} \theta^{(N-q+s+r+d+1)}(s) \\
& \quad=A\left\{\sum_{i=0}^{N+1}\left(\left(\left|\lambda_{0}\right|+\left|\lambda_{0}-\Lambda_{0}\right|\right)\left|t_{0}\right|\right)^{i} / i !\right\} \theta^{(N+1)}(s) .
\end{aligned}
$$

So

$$
\begin{aligned}
& \lambda^{k p-1} G_{q}^{p-1}(z, \partial, \lambda, \tau) v_{N-q}(z, t, \lambda) \\
& \quad \ll A B^{N}\left\{\sum_{i=0}^{N+1}\left(\left(\left|\lambda_{0}\right|+\mid \lambda_{0}-\Lambda_{0} i\right)\left|t_{0}\right|\right)^{i} / i !\right\} \theta^{(N+1)}(s) .
\end{aligned}
$$

Since $\lambda^{k} p^{-1} G_{0}^{p-1}(z, \lambda, 0)^{-1} \ll A\left(R^{\prime}-s\right)^{-1}$, we have (7.27) for $n=N$. Thus

$$
\left|\lambda_{0}^{k} p-1 v_{n}\left(z, t, \lambda_{0}\right)\right| \leqq A B^{n+1}\left(\sum_{i=0}^{n+1}\left(\left(\left|\lambda_{0} t_{0}\right|\right)^{i} / i !\right)(n+1) !\right.
$$

for a small neighborhood of $z=0$.

We proceed to the proof of Proposition 6.8. In this case $i=1$ and 


$$
\begin{aligned}
& G_{0}(z, \lambda, \tau)=G_{0}^{1}(z, \lambda, \tau) \\
& \quad=\lambda^{m-\left(1-a_{1}\right) d_{m}} \sum_{k, l} \lambda^{-\beta_{k, l}^{1}\left(-\alpha z_{1}\right)^{\jmath(k, l)} \tau^{d_{k, l}} a_{k, l}(\tau) .}
\end{aligned}
$$

From Proposition 6.4 we have

Lemma 7.5. It holds that in $\tau(i)(0 \leqq i \leqq p-1)$

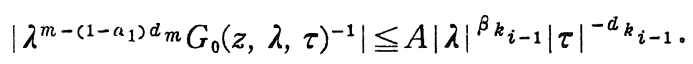

Lemma 7.6. There exists a constant $A$ such that for $\tau \in \tau(i)$

$$
\left|\frac{\lambda^{m-\left(1-a_{1}\right) d_{m}-\left(1-a_{1}\right) r-\beta_{k, l}^{1}} \tau^{r+d_{k, l}}}{G_{0}(z, \lambda, \tau)}\right| \leqq A .
$$

Proof. We have on $\left\{|\tau|=b_{i-1}|\lambda|^{a_{i-1}-\alpha_{1}}\right\}$

$$
\mid \lambda^{-\left(1-\alpha_{1}\right) r-\beta_{k, l}^{1}}\left(\left.\tau^{r+d_{k, l}}|\leqq C| \lambda\right|^{-\left(1-\alpha_{1}\right) r-\beta_{k, l}^{1}-\left(r+d_{k} l\right)\left(\alpha_{1}-\alpha_{i-1}\right)} .\right.
$$

Since

$$
\begin{aligned}
& \left(1-\alpha_{1}\right) r+\beta_{k, l}^{1}+\left(r+d_{k, l}\right)\left(\alpha_{1}-\alpha_{i-1}\right) \\
& \quad=\left(1-\alpha_{i-1}\right) r+\left(\alpha_{1}-\alpha_{\imath-1}\right) d_{k, l}+\beta_{k_{,} l}^{1} \geqq \beta_{k_{i-1}}^{1}+\left(\alpha_{1}-\alpha_{i-1}\right) d_{k_{i-1}},
\end{aligned}
$$

we have

$$
\left|\lambda^{-\left(1-\alpha_{1}\right) r-\beta_{k, l}^{1}} \tau^{r+d_{k, l}}\right| \leqq C|\lambda|^{-\beta_{k_{i-1}}^{1}|\tau|^{d_{k_{i-1}}}} .
$$

On the other hand we have

$$
\left(1-\alpha_{i}\right) r+\left(\alpha_{1}-\alpha_{i}\right) d_{k, l}+\beta_{k, l}^{1} \geqq\left(\alpha_{1}-\alpha_{\imath}\right) d_{k, l}+\beta_{k_{,} l}^{1} \geqq \beta_{k_{i-1}}^{1}+\left(\alpha_{1}-\alpha_{i}\right) d_{k_{i-1}}
$$

Hence we have

on

$$
\left|\lambda^{-\left(1-\alpha_{1}\right) r-\beta_{k, l}^{1}} \tau^{r+d_{k, l}}\right| \leqq C|\lambda|^{-\beta k_{i-1}^{1}|\tau|^{d_{k_{i-1}}}}
$$

$$
\left\{|\tau|=b_{i} \mid \lambda_{1}^{\alpha_{i}-\alpha_{1}}\right\} \cup\left\{\lambda^{a_{i}-\alpha_{1}} \partial K_{i}(\eta)\right\} \text {. }
$$

It follows from Lemma 7.5 that (7.31) holds on the boundary of $\tau(i)$. By the maximal principle of holomorphic functions implies (7.31) holds on $\tau(i)$.

Proof of Proposition 6.8. We show

$$
\begin{aligned}
& \lambda^{m-\left(1-\alpha_{1}\right) d m}\left(\tau-\tau_{1}\right)^{m} w_{n}^{h}(z, t, \lambda, \tau) \\
& \quad \ll A\left(\frac{B}{|\tau|}\right)^{n+1}\left\{\sum_{i=0}^{n+1}\left(\left(\left|\lambda_{0}\right|+\left|\lambda_{0}-\Lambda_{0}\right|\right)\left|t_{0}\right|\right)^{i} / i !\right\} \theta^{(n+1)}(s), \quad h \neq m,
\end{aligned}
$$

where $\theta(s)=\left(R^{\prime}-s\right)^{-1}$ and $s=z_{0}+z_{1}+\cdots+\left(\left(\lambda-\lambda_{0}\right) /\left(\left|\lambda_{0}-\Lambda_{0}\right|\right)\right)$. (7.32) is true for $n=-1$. Assume (7.32) for $\tau \in \tau(i)$ and $-1 \leqq n \leqq N-1$. Since $a_{k, l, s}\left(z, \partial^{\prime}\right) \partial_{0}{ }^{r}$ is эn operator with the order $\leqq s+r$, from Lemma 7.3, 


$$
\begin{aligned}
& \lambda^{m-\left(1-\alpha_{1}\right) d_{m}} a_{k, l, s}\left(z, \partial^{\prime}\right) \partial_{0}^{r} w_{N-q}^{h}(z, t, \lambda, \tau) \\
& \quad \ll A\left(\frac{B}{|\tau|}\right)^{n-q+1}\left\{\sum_{i=0}^{N-q+s+r+1}\left(\left(\left|\lambda_{0}\right|+\left|\lambda_{0}-\Lambda_{0}\right|\right)\left|t_{0}\right|\right)^{i} / i !\right\} \theta^{(N-q+s+r+1)}(s) .
\end{aligned}
$$

By the same method as the proof of Proposition 4.1, we have

$$
\begin{gathered}
\lambda^{m-\left(1-a_{1}\right) d_{m}} Q_{i+r, d}^{j, k}\left(n+s+r, \lambda t_{0}-\lambda \partial_{\lambda}\right) a_{k, l, s}\left(z, \partial^{\prime}\right) \partial_{0}{ }^{r} w_{N-q}^{h}(z, t, \lambda, \tau) \\
\ll A\left(\frac{B}{|\tau|}\right)^{n-q+1}\left\{\sum_{i=0}^{N+1}\left(\left(\left|\lambda_{0}\right|+\left|\lambda_{0}-\Lambda_{0}\right|\right)\left|t_{0}\right|\right)^{i} / i !\right\} \theta^{(N+1)}(s) .
\end{gathered}
$$

Thus by Lemma 7.6 we have $(7.32)$ for $n=N$. Since $\theta^{(n)}(s)=n ! /\left(R^{\prime}-s\right)^{n+1}$, we have (6.41). By the same method, we can show

$$
\begin{aligned}
& \lambda^{m-\left(1-\alpha_{1}\right) d_{m}} \tau w_{n}^{m}(z, t, \lambda, \tau) \\
& \ll A\left(\frac{B}{|\tau|}\right)^{n+1}\left\{\sum_{i=0}^{n+1}\left(\left(\left|\lambda_{0}\right|+\left|\lambda_{0}-\Lambda_{0}\right|\right)\left|t_{0}\right|\right)^{i} / i !\right\} \theta^{(n+1)}(s)
\end{aligned}
$$

and we have (6.42).

\section{References}

[1] Hamada, Y., The singularities of solutions of Cauchy problem, Publ. RIMS. Kyoto Univ., 5 (1969), 21-40.

[2] Hamada, Y., Leray, J. et Wagschal, C., Systèmes d'équations aux derivées partielles à caractéristiques multiples; problème de Cauchy ramifié; hyperbolicité partielle, J. Math. Pures Appl., 55 (1976), 297-352.

[3] Kashiwara, M. and Schapira, P., Problème de Cauchy pour les systèmes microdifférentiels dans le domain complexe, Inv. Math., 46 (1978), 17-38.

[4] Komatsu, H., A local version of Bochner's tube theorem, J. Fac. Sci. Univ. Tokyo, Sect., IA 19 (1972), 201-214.

[5] - Irregularity of characteristic elements and construction of null solutions, J. Fac. Sci. Univ. Tokyo, Sect., IA 23 (1976), 297-342.

[6] Ouchi, S., Asymptotic behavior of singular solutions of linear partial differential equations in the complex domain, J. Fac. Sci. Univ. Tokyo Sect., IA 27 (1980), $1-36$.

[7] - An integral representation of singular solutions of linear partial differential equations in the complex domain, J. Fac. Sci. Univ. Tokyo Sect., IA 27 (1980), 37-85.

[8] - Characteristic Cauchy problems and solution of formal power series, Ann. Inst. Fourier, 33 (1983), 131-176.

[9] - Index, localization and classification of characteristic surfaces for linear partial differential operators, Proc. Jap. Acad., 60A (1984), 189-192.

[10] - Existence of singular solutions and null solutions for linear partial differential operators, J. Fac. Sci. Univ. Tokyo Sect., IA 32 (1985), 457-498.

[11] - The behaviour near the characteristic surface of singular solutions of linear partial differential equation in the complex domain, Proc. Jap. Acad., 65A (1989), 102-105.

[12] —, An integral representation of singular solutions and removable singularities of solutions to linear partial differential equations, Publ. RIMS, Kyoto 
Univ., 26 (1990), 735-783.

[13] Persson, J., Singular holomorphic solutions of linear partial differential equations with holomorphic coefficients and nonanalytic solutions of equations with analytic coefficients, Astérisque 89-90, analytic solutions of partial differential equations (Trento 1981), Soc. Math. France, 223-247.

[14] Ramis, J.P., Dévissage Gevrey, Astérisque 59-60, Soc. Math France, (1978) 173-204.

‘15] Wagschal, C., Problème de Cauchy analytique à données méromorphes, J. Math. Pures Appl., 51 (1972), 375-397. 